\author{
Universidade de São Paulo \\ Instituto de Astronomia, Geofísica e Ciências Atmosféricas
}

Departamento de Astronomia

Felipe Andrade Oliveira

\title{
Reduzindo o Setor Escuro do Universo: Uma Nova Cosmologia Acelerada com Criação de Matéria Escura Fria
}

São Paulo 

Felipe Andrade Oliveira

\section{Reduzindo o Setor Escuro do Universo: Uma Nova Cosmologia Acelerada com Criação de Matéria Escura Fria}

Dissertação apresentada ao Departamento de Astronomia do Instituto de Astronomia, Geofísica e Ciências Atmosféricas da Universidade de São Paulo como parte dos requisitos para a obtenção do título de Mestre em Ciências.

Área de Concentração: Astronomia

Orientador: Prof. ${ }^{o}$ Dr. ${ }^{o}$ José Ademir de Sales Lima

São Paulo 

À minha família e aos meus amigos 



\section{Agradecimentos}

Ao professor José Ademir Sales de Lima, pela sugestão do tema, pela orientação e por dividir sua grande experiência.

A José Fernando de Jesus, pela amizade e colaboração em um trabalho.

Ao grupo de cosmologia: Antonio C. C. Guimarães, Carlos H. G. Bessa, João Maria da Silva, João Vital da Cunha, José Fernando de Jesus, Rodrigo Fernandes Holanda, Rose Clívia Santos e Vinícius Consolini Busti, por todas as discussões científicas e pela amizade.

À minha família, principalmente aos meus pais, Maria Silvana Andrade da Silva e Estevan Antônio de Oliveira Neto, meu irmão Leandro Andrade Oliveira e meu afilhado Murilo Andrade Ribeiro Guedes.

À minha namorada Aline Cristina Nunes de Almeida, por todo apoio, paciência e muito amor.

A todos meus inestimáveis amigos do CEFET-Cubatão, do IFUSP e do IAG-USP.

Aos professores do Departamento de Astronomia do IAG, principalmente aos professores Jane C. Gregorio Hetem, Nelson Vani Leister, Antônio Mário Magalhães, Roberto Dias da Costa, Eduardo Serra Cypriano, Laerte Sodré Júnior, Sílvia Rossi, Ronaldo Eustáquio de Souza, Gastão Lima Neto pelas disciplinas lecionadas e por toda a atenção dada.

Aos funcionários do IAG, por toda a assistência prestada.

Aos membros da Representação Discente, especialmente ao Oscar Cavichia de Moraes e ao Vinicius Moris Placco pelo desenvolvimento da classe IAGTESE.

Ao Departamento de Astronomia (IAG-USP), por propiciar um ótimo ambiente de trabalho e pesquisa.

Ao CNPq, pelo apoio financeiro, sob o projeto $\mathrm{N}^{o}:$ 134266/2008-0. 


\footnotetext{
${ }^{1}$ Esta dissertação foi escrita em LATEX com a classe IAGTESE, para teses e dissertações do IAG.
} 
"Tudo, aliás, é a ponta de um mistério, inclusive os fatos. Ou a ausência deles. Duvida? Quando nada acontece há um milagre que não estamos vendo. " 



\section{Resumo}

Nesta dissertação nós propomos uma nova cosmologia relativística acelerada cujo conteúdo material é composto apenas por bárions e matéria escura fria. A não existência de uma componente de energia escura implica que nosso cenário é baseado numa redução do chamado setor escuro do universo.

Neste modelo, o presente estágio acelerado é determinado pela pressão negativa descrevendo a produção de partículas de matéria escura fria induzida pelo campo gravitacional variável do universo. Para um universo espacialmente plano $\left(\Omega_{d m}+\Omega_{b}=1\right)$, como previsto pela inflação, este tipo de cenário possui somente um parâmetro livre e a equação diferencial governando a evolução do fator de escala é exatamente a mesma do modelo $\Lambda$ CDM. Neste caso, encontramos que o parâmetro efetivo de densidade de matéria é $\Omega_{m e f f}=1-\alpha$, onde $\alpha$ é um parâmetro constante ligado à taxa de criação de matéria escura fria.

Aplicando um teste estatístico $\chi^{2}$ para os dados de Supernovas do tipo Ia (Union Sample 2008), limitamos os parâmetros livres do modelo nos casos espacialmente plano e com curvatura. Em particular, encontramos que para o caso plano $\alpha \sim 0.71$, de forma que $\Omega_{\text {meff }} \sim 0.29$, como tem sido inferido independentemente por lentes gravitacionais fracas, estrutura de grande escala, radiação cósmica de fundo e outras observações complementares. 



\section{Abstract}

In this dissertation we propose a new accelerating relativistic cosmology whose matter content is composed only by baryons and cold dark matter. The nonexistence of a dark energy component implies that our scenario is based on a reduction of the so-called dark sector of the Universe.

The present accelerating stage in this model is powered by the negative pressure describing the cold dark matter particle production induced by the variable gravitational field of the Universe. For a spatially flat universe $\left(\Omega_{d m}+\Omega_{b}=1\right)$, as predicted by inflation, this kind of scenario has only one free parameter and the differential equation governing the evolution of the scale factor is exactly the same of the $\Lambda$ CDM model. In this case, we find that the effectively observed matter density parameter is $\Omega_{\text {meff }}=1-\alpha$, where $\alpha$ is a constant parameter related to the cold dark matter creation rate.

By applying a $\chi^{2}$ statistical test for Supernovae type Ia data (Union Sample 2008), we constrain the free parameters of the model for spatially flat and curved cases. In particular, to the flat case we find $\alpha \sim 0.71$, so that $\Omega_{\text {meff }} \sim 0.29$, as independently inferred from weak gravitational lensing, large scale structure, cosmic background radiation, and other complementary observations. 



\section{Notação e Convenções}

- Assinatura da métrica: (+,-,-,-).

- Índices gregos variam de 0 a 3 , índices latinos variam de 1 a 3 . Índices repetidos obedecem à convenção de Einstein.

- Derivada parcial: $\frac{\partial \phi}{\partial x^{\alpha}} \equiv \phi, \alpha$.

- Derivada covariante: $A^{\alpha}{ }_{\beta}=A^{\alpha}{ }_{\beta}+\Gamma_{\lambda \beta}^{\alpha} A^{\lambda}$.

- Salvo menção contraria, usaremos um sistema de unidades onde c=1.

- Expressões em outros idiomas serão escritas em itálico. 



\section{Informação Eletrônica}

A maioria das referências bibliográficas utilizadas nessa dissertação podem ser encontradas nas seguintes páginas da WEB:

- http://www.periodicos.capes.gov.br/

- http://adsabs.harvard.eduabstract_service.html

- http://xxx.lanl.gov/ 



\section{Lista de Figuras}

1.1 Anisotropias de temperatura da Radiação Cósmica de Fundo . . . . . . . . 44

1.2 Curva de rotação da galáxia NGC6503. . . . . . . . . . . . . . . . . . 45

1.3 Evidência de matéria escura no Bullet Cluster . . . . . . . . . . . . . . 47

1.4 Evidência da aceleração do universo obtida pelo Supernova Cosmology Project (Perlmutter, 1999). . . . . . . . . . . . . . . 51

1.5 Evidência da aceleração do universo obtida pelo High-z Supernova Search (Riess et al., 1998). . . . . . . . . . . . . . . . . . . 52

2.1 Genealogia da energia escura . . . . . . . . . . . . . . . 54

2.2 Plano $\left(\Omega_{\Lambda} \times \Omega_{m}\right) \ldots \ldots \ldots \ldots$

2.3 Plano $\left(\Omega_{M} \times \omega\right) \ldots \ldots \ldots$. . . . . . . . . . . . . 60

4.1 Plano $\left(\Omega_{m e f f} \times \alpha\right)$ e plano $\left(\Omega_{m} \times \alpha\right)$ para o modelo CCDM . . . . . . . 83

4.2 Likelihood do parâmetro livre $\alpha$ para o modelo CCDM plano . . . . . . . 83

4.3 Likelihood do redshift de transição para o modelo CCDM . . . . . . . . . . 85 



\section{Lista de Tabelas}

1.1 Abundância dos elementos produzidos na Nucleossíntese Primordial . . . . 41

2.1 Modelo ACDM: Parâmetros cosmológicos do modelo e intervalos de confiança de $68 \%$ (Komatsu et al., 2010). . . . . . . . . . . . . 55

4.1 Modelo CCDM . . . . . . . . . . . . . . . . . . . 84

5.1 Modelo $\Lambda$ CDM vs. Modelo CCDM . . . . . . . . . . . . . 88 



\section{Sumário}

1. Cosmologia Relativística . . . . . . . . . . . . . . . . . . . . . . . 29

1.1 Teoria da Relatividade Geral . . . . . . . . . . . . . . . . . . . 29

1.2 Cosmologia Relativística - Modelos de Big Bang . . . . . . . . . . . . . . 33

1.2.1 Métrica de Friedmann-Robertson-Walker . . . . . . . . . . . . . . 33

1.2.2 A Expansão do Universo . . . . . . . . . . . . . . . . . 34

1.2.3 Modelos Cosmológicos do tipo Friedmann . . . . . . . . . . . . . 35

1.2.4 Parâmetros Cosmológicos Básicos . . . . . . . . . . . . 36

1.2.5 Redshift . . . . . . . . . . . . . . . . . 38

1.3 Modelos do Big Bang: Base Observacional . . . . . . . . . . . . . . . . 39

1.3.1 Nucleossíntese Primordial . . . . . . . . . . . . . . . . . . . 39

1.3.2 Radiação Cósmica de Fundo . . . . . . . . . . . . . . . . . . 41

1.4 Modelo de Concordância Cósmica . . . . . . . . . . . . . . . . . . . 43

1.4.1 Matéria Escura . . . . . . . . . . . . . . . 43

1.4.2 Distâncias Cosmológicas . . . . . . . . . . . . . . . 48

1.4.3 Supernovas e Aceleração do Universo . . . . . . . . . . . . . . . 50

2. Modelos de Energia Escura . . . . . . . . . . . . . . . . . . . . 53

2.1 O Termo $\Lambda \ldots \ldots \ldots \ldots \ldots$

2.2 Decaimento do Vácuo $(\Lambda(t)) \ldots \ldots . \ldots . \ldots$

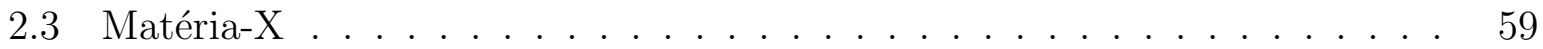

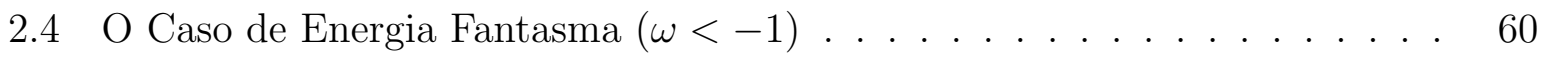

2.5 Campo Escalar - Quintessência . . . . . . . . . . . . . 62 
2.6 Gás Tipo Chaplygin . . . . . . . . . . . . . . . . . . . . . . . . 64

2.7 Gás de Chaplygin Simplificado . . . . . . . . . . . . . . . . . . . 65

3. Modelos Acelerados com Criação de Matéria . . . . . . . . . . . . . . . . . . . . 69

3.1 Criação de Matéria Escura no Universo: Formulação Termodinâmica . . 70

3.2 Dinâmica da Criação de Matéria Escura . . . . . . . . . . . . . . . . 72

3.3 Lei de Evolução da Temperatura . . . . . . . . . . . . . . . . . . . . 74

4. Um Novo Modelo com Criação de Matéria . . . . . . . . . . . . . . . . . 77

4.1 Modelo CCDM (Creation Cold Dark Matter) _ . . . . . . . . . . 78

4.2 Vínculos de Supernovas e Redshift de Transição . . . . . . . . . . . . . . . 81

4.3 Comentários Finais . . . . . . . . . . . . . . . . . . . . . . 84

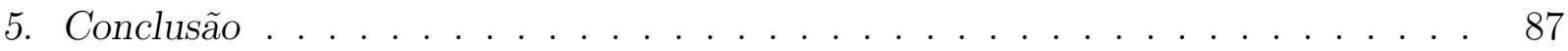

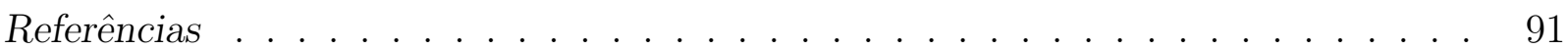




\section{Introdução}

A Cosmologia é a área da Física que estuda o universo em grande escala, sua origem, evolução e composição. A história da Cosmologia moderna começa com a criação da Teoria da Relatividade Geral (TRG) por A. Einstein, em 1915. A TRG é a teoria que melhor descreve a interação gravitacional (Will, 2005). Como é bem conhecido, com base nesta teoria se obtém uma descrição mais precisa do universo, ou seja, livre das contradições introduzidas pela gravitação newtoniana.

Em Cosmologia, o conhecimento sobre o universo é expresso através dos chamados modelos cosmológicos ou modelos de universo. Tais modelos são criados a partir de leis e princípios básicos e devem ser sustentados pelas observações astronômicas. A hipótese mais fundamental utilizada na construção dos modelos de universo é o chamado Princípio Cosmológico. Tal princípio estabelece que o Universo é homogêneo e isotrópico, ou melhor, não existe nem local nem direção privilegiada, independentemente da posição ocupada pelo observador.

O primeiro modelo cosmológico baseado na TRG e no Princípio Cosmológico foi proposto por Einstein (1917). Na época, a idéia geral era de que o universo deveria ser estático. Para produzir um modelo coerente com esta crença geral, Einstein precisou introduzir um termo adicional nas equações de campo para contrabalancear o efeito atrativo da gravitação. Pelas circunstâncias em que foi introduzido, este termo constante foi batizado como constante cosmológica.

Outros modelos não tardaram a aparecer. Imaginando que a quantidade de matéria do universo pudesse ser desprezível, W. de Sitter (1917) obteve uma solução para as equações de campo de Einstein com o universo estático e constituído apenas por constante cosmológica. 
No início da década de 1920, A. A. Friedmann percebeu que as equações de campo, para um universo homogêneo e isotrópico, permitiam soluções dinâmicas com $\Lambda=0$ e $\Lambda \neq 0$. Foram então obtidas as soluções cosmológicas com curvatura espacial positiva (Friedmann, 1922) e com curvatura negativa (Friedmann, 1924).

A credibilidade de modelos expansionistas se acentuou a partir das observações de E. Hubble (1929). Hubble observou que a radiação proveniente de galáxias apresentava um desvio sistemático para o vermelho (redshift), proporcional à distância em que cada galáxia se encontrava. Esta relação, conhecida como lei de Hubble, é bem explicada pelos modelos expansionistas de Friedmann, cujo o afastamento entre as galáxias é interpretado como a própria expansão do espaço entre elas.

A evidência da expansão cosmológica levou Einstein a abandonar definitivamente a idéia de universo estático. Em colaboração com de Sitter (Einstein e de Sitter, 1932), estes estudaram o modelo de universo caracterizado por constante cosmológica nula, pela seção espacial plana e por matéria não-relativística, conhecido na literatura como modelo de Einstein-de Sitter (EdS). Algumas propriedades dos modelos de Friedmann e do modelo EdS foram estudadas por G. Lemaitre (1934) e classificadas do ponto de vista de simetrias das seções espaciais por H. Robertson (1936) e A. Walker (1936), sendo denominadas na literatura como modelos de Friedman-Robertson-Walker (FRW). A classe de modelos EdS constituía até recentemente o chamado modelo cosmológico padrão.

Tomando os modelos expansionistas como base, Gamow e colaboradores (Gamow, 1946; Alpher et al., 1948) investigaram a possibilidade da produção dos elementos químicos no universo primordial. Contudo, a instabilidade dos núcleos com massa 5 e 8 não permitiam que elementos mais pesados que o berílio pudessem ser formados neste cenário. Este problema só foi posteriormente contornado por Burbidge, Burbidge, Fowler e Hoyle (1957), através da proposição de que os elementos mais pesados teriam origem na nucleossíntese estelar.

Em seus estudos sobre a nucleossíntese, Gamow ainda previu que o universo deveria ser permeado por um campo de radiação homogêneo, isotrópico e com espectro característico de um corpo negro (Alpher et al., 1948). Este fundo de radiação, remanescente de uma fase extremamente quente do universo primordial, voltou a ser estudado por Dicke, Peebles, Roll e Wilkinson (1965) e, no mesmo ano, foi independentemente observado por Penzias e 
Wilson (1965) como um "excesso de temperatura de antena". Esta observação deu forte sustentação à hipótese do Hot Big-Bang que, na época, tinha como principal rival a teoria de estado estacionário (Hoyle, 1948).

Até o final dos anos 90, o modelo de Einstein-de Sitter era considerado o modelo padrão de Cosmologia. Porém, em 1998, através das observações de Supernovas do Tipo Ia (SNe Ia), dois grupos descobriram, independentemente, que o universo passa por uma fase de expansão acelerada (Riess et al., 1998; Perlmutter, 1999). Este resultado surpreendeu a comunidade científica pois, como a gravidade é uma força atrativa, esperava-se observar um universo em expansão desacelerada. Tal descoberta tem impacto comparável à detecção da Radiação Cósmica de Fundo por Penzias e Wilson (1965), abrindo novas perspectivas sobre a nossa compreensão do universo.

A evidência de aceleração, fornecida pelos dados de Supernovas do tipo Ia, nos leva a inferir a existência de uma componente escura com pressão negativa, genericamente batizada de energia escura. A existência deste termo é compatível com outras observações, entre as quais podemos citar: as anisotropias do espectro de potência da radiação cósmica de fundo (Komatsu et al., 2010), estruturas em grande escala (Cole et al., 2005; Tegmark et al., 2004), observações em raios X de aglomerados de galáxias (Lima et al., 2003), objetos velhos em altos redshifts (Krauss, 1997; Alcaniz e Lima, 1999), tamanho angular de fontes de rádio compactas (Gurvits et al., 1999; Lima e Alcaniz, 2002) e rádio-galáxias (Daly e Guerra, 2002), gamma-ray bursts (Schaefer, 2007).

O atual modelo cosmológico padrão é representado pelo modelo $\Lambda \mathrm{CDM}$, cujas componentes dominantes são a matéria escura fria e a energia do vácuo (constante cosmológica). Devido ao bom ajuste fornecido aos dados observacionais, é também chamado de modelo de concordância cósmica. Porém, este modelo não é perfeito, apresentando vários problemas e algumas inconsistências teóricas. Uma questão muito abordada na literatura recente diz respeito à incompatibilidade entre os valores estimados observacionalmente e pela teoria quântica de campos para a constante cosmológica. A discrepância entre estas duas estimativas chega a 120 ordens de grandeza, caracterizando o chamado Problema da Constante Cosmológica (PCC).

O PCC, assim como outras incoerências no modelo padrão, tem estimulado a produção de muitos modelos alternativos capazes de explicar o período acelerado em que o universo 
se encontra:

(i) Dentro do cenário relativístico, podemos citar os modelos em que a densidade de energia do vácuo varia com o tempo, os modelos $\Lambda(t) \mathrm{CDM}$; a matéria -X, cuja a equação de estado é $p=\omega \rho$ para $\omega<0$; um campo escalar que decai lentamente; o gás de Chaplyging, descrito pela equação de estado $p=-A / \rho^{\alpha}$, onde $A$ e $\alpha$ são constantes positivas, entre outros.

(ii) Já nos cenários construídos fora da gravitação de Einstein, podemos destacar as teorias que modificam a ação de Einstein-Hilbert, os modelos criados dentro da teoria de branas, os cenários inomogêneos e os cenários holográficos.

Tanto nos cenários relativísticos como nos alternativos à TRG, os espaços de parâmetros obtidos costumam ser muito degenerados e, muitas vezes, quando confrontados com as observações, revelam ter problemas e incoerências.

Uma das fontes de dificuldades encontrada neste tipo de investigação é que, na verdade, esta componente não pôde ser identificada. Não existem ainda evidências diretas de sua existência ou de seus efeitos dinâmicos. Em síntese, enquanto a aceleração é um fato bem estabelecido, o mesmo não ocorre com a questão sobre a existência da energia escura. Neste caso, modelos sem a presença deste termo são alternativas que devem ser seriamente consideradas.

Do ponto de vista observacional, um bom modelo cosmológico deve satisfazer aos seguintes requisitos: (i) ser aproximadamente plano, como indicam os dados relativos à radiação cósmica de fundo, (ii) possuir uma componente de matéria escura não-relativística e não-bariônica, requerido pelas curvas de rotação das galáxias, espectro de potência da matéria e dados obtidos de fusões de aglomerados, (iii) sua fase atual ser de expansão acelerada, de acordo com as observações de Supernovas do tipo Ia e, por último, (iv) ser capaz de compatibilizar uma constante de Hubble, $H_{0} \approx 72 \mathrm{~km} \cdot \mathrm{s}^{-1} \cdot M p c^{-1}$, com a previsão de que o universo deve ter idade superior a 12 bilhões de anos, para que acomode as estruturas mais antigas observadas.

Os estudos desenvolvidos nesta dissertação estão contidos neste contexto geral. Estamos propondo um novo cenário cosmológico acelerado, onde a aceleração cósmica é devida unicamente à criação de partículas de matéria escura fria. Neste caso, o setor escuro do universo fica reduzido a apenas uma componente, a saber, a matéria escura fria, cujo status 
relativo é bem maior que o da energia escura.

Mostramos também que o modelo proposto é consistente com os dados de Supernova do tipo Ia não havendo necessidade que o parâmetro de Hubble seja pequeno para resolver o problema da idade total e em altos redshifts do universo. O trabalho original desta dissertação (apresentado no capítulo 4) está submetido à publicação e é citado no texto como (Lima et al., 2009).

Em linhas gerais, a estrutura e o desenvolvimento do corpo desta dissertação é descrito a seguir.

O primeiro capítulo se inicia com uma breve revisão da Teoria da Relatividade Geral, passando por seus conceitos básicos e as principais expressões de interesse cosmológicos. Nesse capítulo, também introduzimos os principais modelos cosmológicos, mostrando deduções e discussões simplificadas das equações que descrevem a dinâmica e a evolução dos modelos de universo. Discutimos também o modelo padrão de cosmologia e apresentamos as principais bases observacionais do modelo de Big Bang.

No segundo capítulo discutimos as diversas alternativas dos modelos cosmológicos que se propõem a explicar a aceleração do universo. Entre as alternativas, mostramos que é possível acelerar o universo através (i) da inclusão de algum fluido exótico com pressão negativa, (ii) de modificações na própria teoria da Relatividade Geral, ou ainda (iii) através dos mecanismos da criação de matéria escura.

No capítulo 3, nos aprofundamos no cenário com criação cosmológica de matéria e sua formulação macroscópica. Mostramos que o processo de criação de partículas de matéria escura é um processo irreversível que afeta as equações de campo do universo através de um termo de pressão negativa que pode ser responsável pela aceleração do universo.

No quarto capítulo, apresentamos uma nova proposta de modelos com criação cosmológica de matéria escura: o modelo batizado como CCDM (Creation Cold Dark Matter). Neste capítulo, estudamos em detalhes suas equações e resultados. Realizamos o teste de $\chi^{2}$ para os dados de Supernova Tipo Ia, vinculando os valores dos parâmetros livres do modelo nos casos plano e com curvatura. Mostramos que o modelo permite a transição entre a expansão desacelerada e acelerada. Destacamos ainda que, apesar das hipóteses iniciais distintas, existe uma equivalência completa entre as dinâmicas do modelo CCDM e do modelo padrão $\Lambda C D M$. 
Finalmente apresentamos nossas conclusões e descrevemos algumas das perspectivas futuras do nosso trabalho. 


\section{Capítulo 1}

\section{Cosmologia Relativística}

O universo pode ser descrito como um fluido auto-gravitante e, portanto, no seu estudo devemos adotar uma teoria para descrever a interação gravitacional. As melhores teorias gravitacionais existentes são a de Einstein e a de Newton. A teoria de Einstein é uma versão relativística da teoria de Newton e está de acordo com todos os dados observacionais dispostos até o presente e, portanto, será adotada nesta dissertação (Will, 2005).

Neste capítulo são mostradas as bases da teoria da relatividade geral e da cosmologia relativística que serão usadas ao longo de todo o trabalho mostrado. O conteúdo exposto aqui pode ser encontrado de forma mais detalhada em livros textos de relatividade geral e cosmologia, como por exemplo em Weinberg (1972), Weinberg (2008), Durrer (2007), Peacock (1999), entre outros.

\subsection{Teoria da Relatividade Geral}

A Teoria da Relatividade Geral (TRG) foi proposta por Albert Einstein em 1915 como uma extensão da Teoria da Relatividade Restrita na qual os efeitos provocados por campos gravitacionais foram incorporados. O passo crucial para a criação da teoria da Relatividade Geral foi dado por Einstein em 1907 através do chamado princípio da equivalência entre gravitação e inércia ou simplesmente princípio da equivalência (PE).

Desde os experimentos de Galileo Galilei no período entre 1602 e 1604 é conhecido o fato de que todos os corpos na superfície da Terra se movem sob a mesma aceleração, independentemente de suas massas. Com o posterior surgimento da mecânica e da gravitação Newtoniana este fato pôde ser interpretado através da equivalência entre a massa inercial $\left(m_{I}\right)$ e a massa gravitacional $\left(m_{G}\right)$, embora o próprio Isaac Newton chamasse a 
atenção para o fato de que tais massas não precisassem ser obrigatoriamente iguais. Em 1889, Roland Eötvös mostrou que se $m_{G}$ e $m_{i}$ fossem diferentes, tal discrepância deveria ser inferior a $10^{-9}$; experimentos mais recentes mostram que $1-\frac{m_{G}}{m_{I}} \leq 10^{-14}$ (Turyshev, 2008).

Partindo desta equivalência entre massas, Einstein admitiu que seria impossível distinguir localmente entre a aceleração de origem gravitacional e a aceleração causada por um referencial acelerado. Assim, segundo a TRG, a força da gravidade ocupa um lugar especial dentre as forças fundamentais da natureza, visto que, para quaisquer partículas de teste sujeitas às mesmas condições iniciais, independentemente das suas propriedades (incluindo suas massas de repouso) o efeito observado sobre estas será o mesmo. Em síntese, no contexto da TRG, o comportamento de um corpo de teste sob a ação de um campo gravitacional é interpretado como um efeito puramente geométrico.

Utilizando o PE, em 1907 Einstein calculou o desvio para o vermelho sofrido pela luz sujeita a um campo gravitacional, mostrando então que era possível calcular o efeito causado pela gravidade sobre um sistema físico arbitrário. Porém, para a formulação final da TRG, ainda era necessário determinar como são formados os campos gravitacionais. Em colaboração com o matemático Marcel Grossman, em 1913 Einstein associou o campo gravitacional a 10 componentes do tensor métrico do espaço-tempo de Riemann, resultando na forma tensorial da teoria de gravitação que conhecemos hoje.

A geometria de um sistema físico é determinada através de sua métrica. Na relatividade restrita, o elemento de linha pseudo-euclidiano é dado por $(c=1)$

$$
d s^{2}=d t^{2}-d x^{2}-d y^{2}-d z^{2}
$$

com $t$ a coordenada temporal e $x, y$ e $z$ as coordenadas espaciais. De modo sucinto, a relação acima pode ser expressa como

$$
d s^{2}=\eta_{\mu \nu} d x^{\mu} d x^{\nu}
$$

onde $\eta_{\mu \nu} \equiv$ Diagonal(+---) é o chamado tensor métrico do espaço-tempo de Minkowski.

Na TRG, a geometria é modificada de acordo com a curvatura induzida pela presença do campo da matéria, com o elemento de linha tomando a seguinte forma (Weinberg, 1972): 


$$
d s^{2}=g_{\mu \nu} d x^{\mu} d x^{\nu}
$$

onde $g_{\mu \nu}$ é o tensor métrico do espaço-tempo curvo arbitrário. De posse do tensor métrico, podemos calcular o tensor de Einstein através da expressão

$$
G_{\mu \nu}=R_{\mu \nu}-\frac{1}{2} g_{\mu \nu} R
$$

onde $R_{\mu \nu} \equiv R_{\mu \alpha \nu}^{\alpha}$, o tensor de Ricci, é obtido através da contração do tensor de RiemannChristoffel (Weinberg, 1972)

$$
R_{\mu \beta \nu}^{\alpha}=\frac{1}{2} g^{\alpha \lambda}\left[\frac{\partial^{2} g_{\lambda \beta}}{\partial x^{\mu} \partial x^{\nu}}-\frac{\partial^{2} g_{\mu \beta}}{\partial x^{\nu} \partial x^{\lambda}}-\frac{\partial^{2} g_{\lambda \nu}}{\partial x^{\mu} \partial x^{\beta}}+\frac{\partial^{2} g_{\mu \nu}}{\partial x^{\lambda} \partial x^{\beta}}\right],
$$

e R é o escalar de curvatura, resultante da contração do tensor de $\operatorname{Ricci}\left(R \equiv R_{\mu}^{\mu}\right)$.

Em síntese, as equações de campo na TRG estabelecem uma relação entre a geometria de um sistema físico e o conteúdo massa-energia

$$
G_{\mu \nu}-\Lambda g_{\mu \nu}=\chi T_{\mu \nu}
$$

onde $T_{\mu \nu}$ é o tensor energia-momento e $\chi \equiv 8 \pi G$ a constante de Einstein (obtida através do limite para campos fracos). O termo $\Lambda$ foi introduzido por Einstein por uma questão relacionada à cosmologia, recebendo então o nome de constante cosmológica.

No caso mais simples, quando a fonte de energia-momento é tratada como um fluido perfeito, o conteúdo energético e material pode ser totalmente caracterizado por duas grandezas termodinâmicas, a saber, a densidade de massa-energia $\rho$ e a pressão isotrópica $p$.

Consideremos um sistema de referência localmente lorentziano com o fluido em repouso (com o TEM no dado sistema identificado por $\tilde{T}^{\mu \nu}$ ) em um dado ponto do espaço-tempo $x$. A componente $\tilde{T}^{00}$ de $\tilde{T}^{\mu \nu}$ neste ponto particular representa a densidade de massa-energia do sistema físico (Weinberg, 1972):

$$
\tilde{T}^{00}=\rho
$$

As componentes $\tilde{T}^{i i}$ representam a pressão isotrópica:

$$
\tilde{T}^{i i}=p
$$


Como estamos considerando um fluido sem condução de calor ou viscosidade, temos ainda que $\tilde{T}^{i 0}=\tilde{T}^{0 j}=0$ assim como com $\tilde{T}^{i j}=0$ sempre que $i \neq j$.

Temos $\eta_{\mu \nu}, u^{\mu}=\delta_{0}^{\mu}$ (observador comóvel) e as quantidades $\rho$ e $p$. A forma manifestamente covariante de Lorentz, cujas as componentes se reduzem aos resultados do referencial lorentziano local é dada por:

$$
\tilde{T}^{\mu \nu}=(\rho+p) u^{\mu} u^{\nu}-p \eta^{\mu \nu}
$$

Através do acoplamento mínimo implementamos os efeitos da gravitação, obtendo (Weinberg, 1972):

$$
T^{\mu \nu}=(\rho+p) u^{\mu} u^{\nu}-p g^{\mu \nu}
$$

O TEM satisfaz a seguinte identidade

$$
T^{\mu \nu} ;{ }_{\nu}=0,
$$

que estabelece a forma local das leis de conservação de energia e de momento independentemente do sistema de coordenadas adotado.

É interessante mencionar que as equações dinâmicas da TRG podem ser derivadas através de um princípio da mínima ação. Neste caso a ação total do sistema será

$$
I=I_{E H}+I_{M}
$$

sendo $I_{M}$ a ação de matéria

$$
I_{M}=\frac{1}{2} \int d^{4} x \sqrt{-g} T^{\mu \nu} g_{\mu \nu}
$$

e $I_{E H}$ a ação de Einstein-Hilbert

$$
I_{E H}=\frac{1}{16 \pi G} \int d^{4} x \sqrt{-g} R
$$

onde $g$ é o determinante do tensor métrico e $R$ o escalar de curvatura.

Pode-se mostrar que a variação infinitesimal da ação total $\delta I \equiv \delta I_{E H}+\delta I_{M}$ em relação a $g^{\mu \nu}$ é (Landau e Lifshitz, 1971; Weinberg, 1972): 


$$
\frac{1}{2 \chi} \int \sqrt{-g}\left[R^{\mu \nu}-\frac{1}{2} g^{\mu \nu} R\right] \delta g_{\mu \nu} d^{4} x+\frac{1}{2} \int \sqrt{-g} T^{\mu \nu} \delta g_{\mu \nu} d^{4} x .
$$

O princípio da mínima ação impõe que a ação total seja estacionária em relação a pequenas variações das variáveis dinâmicas. Neste caso, mostra-se que $I$ será estacionário em relação a variações em $g_{\mu \nu}$ se e somente se a seguinte condição for satisfeita:

$$
R^{\mu \nu}-\frac{1}{2} g^{\mu \nu} R=\chi T^{\mu \nu},
$$

ou seja, a equação de campo de Einstein (equação (1.6)).

\subsection{Cosmologia Relativística - Modelos de Big Bang}

\subsubsection{Métrica de Friedmann-Robertson-Walker}

Na cosmologia moderna, aé usualmente suposto que o universo é espacialmente homogêneo e isotrópico, ao menos quando visto em grandes escalas (da ordem de 100 Megaparsecs). Muito antes das evidências providas por observações astronômicas, como por exemplo a distribuição das galáxias em largas escalas e a quase uniformidade da temperatura da radiação cósmica de fundo, Friedmann utilizou esta hipótese na formulação de seus modelos relativísticos de universo. Tal consideração também está relacionada ao chamado Princípio Cosmológico que pode ser visto como uma extensão do princípio de Copérnico; neste caso, consideramos que o próprio universo não possua lugares com características privilegiadas, tendo a mesma aparência para todos os seus observadores comóveis em cada instante $t$. As propriedades de isotropia e homogeneidade se expressam matematicamente através da métrica Friedmann-Robertson-Walker-(FRW):

$$
d s^{2}=d t^{2}-a^{2}(t)\left(\frac{d r^{2}}{1-k r^{2}}+r^{2} d \theta^{2}+r^{2} \sin ^{2} \theta d \phi^{2}\right),
$$

onde $r, \theta, \phi$ são coordenadas comóveis espaciais. A coordenada $t$ pode ser interpretada com o significado de tempo e tem como função definir o "tempo próprio e a hipersuperfície de simultaneidade para todo o universo" (Robertson, 1929). A quantidade $k$ é uma propriedade intrínseca do universo que determina a sua curvatura (se $k=1$ o universo tem curvatura positiva, se $k=0$ é plano ou com curvatura nula e se $k=-1$ tem curvatura negativa) e $a(t)$ é o fator de escala cósmico responsável pela descrição dinâmica do universo. 


\subsubsection{A Expansão do Universo}

As primeiras soluções expansionistas com base na TRG foram obtidas pelo matemático e meteorologista A. A. Friedmann (1922, 1924).

Em 1929, Edwin P. Hubble anunciou uma das mais importantes descobertas do século XX: a expansão do universo. A conclusão de Hubble se baseou nos seus estudos sobre os espectos de emissão de dezenas de galáxias, rompendo com o ideal de que o universo seria estático, como até então se acreditava.

Em suas observações, Hubble constatou um desvio sistemático para o vermelho de linhas espectrais provenientes galáxias, ou seja, as outras galáxias deveriam estar se afastando de nós. Hubble também percebeu que a velocidade de afastamento das galáxias mantinha uma relação aproximadamente linear com suas distâncias:

$$
v=H_{0} d,
$$

relação conhecida como Lei de Hubble, onde o fator de proporcionalidade entre velocidade de recessão e distância é dada pela constante de Hubble $H_{0}$.

O parâmetro de Hubble, relação que determina a taxa de variação do volume comóvel $V$, é definido pela relação

$$
H(t) \equiv \frac{\dot{a}}{a}=\frac{1}{3} \frac{\dot{V}}{V},
$$

sendo que a constante de Hubble, identificada por $H_{0}$, nada mais é que seu valor atual (em $\left.t=t_{0}\right)$.

Usualmente, a constante de Hubble é normalizada como $H_{0}=h \cdot 100 \mathrm{~km} \cdot \mathrm{s}^{-1} \cdot \mathrm{Mpc}^{-1}$, em que o valor de $h$, medido pelo Hubble Space Telescope, é $h=0.72 \pm 0.08$ (Freedman et al., 2001).

A determinação precisa de $H_{0}$ é um problema fundamental da cosmologia moderna. $\mathrm{O}$ valor de $H_{0}$ tem importância prática e teórica para diversas propriedades astrofísicas de galáxias, aglomerados, entre outros, assim como a precisão de sua determinação tem implicações importantes nas estimativas de parâmetros cosmológicos fundamentais e na própria idade total do universo.

Outro importante aspecto do afastamento das galáxias observado por Hubble diz respeito à origem do universo. Seguindo através da análise das equações de Friedmann, 
prevemos que, de forma geral, o universo teve início em uma singularidade. O afastamento das galáxias constitui o primeiro indício de que o universo se expande e, consequentemente, no passado deveria ter sido menor. Como extrapolação, retrocedendo no tempo cada vez mais temos que as distâncias entre dois pontos do espaço cada vez menores, até o limite da singularidade prevista nos modelos de Friedmann no instante inicial $(t \rightarrow 0)$, na qual a densidade do universo tende a infinito. Uma situação extrema que talvez possa ser evitada por uma teoria quântica da gravitação.

\subsubsection{Modelos Cosmológicos do tipo Friedmann}

Ao assumir a métrica (1.17) e o tensor momento-energia para um fluido perfeito (1.10), as equações de campo de Einstein tomam a forma (Friedmann, 1922, 1924; Weinberg, 1972):

$$
\begin{gathered}
8 \pi G \rho+\Lambda=3 \frac{\dot{a^{2}}}{a^{2}}+3 \frac{k}{a^{2}}, \\
8 \pi G p-\Lambda=-2 \frac{\ddot{a}}{a}-\frac{\dot{a^{2}}}{a^{2}}-\frac{k}{a^{2}},
\end{gathered}
$$

onde o ponto (') representa uma derivação em relação ao tempo. Estas equações são conhecidas como equações de Friedmann e são elas quem regem o comportamento do fator de escala do universo. A constante $\Lambda$, conhecida como constante cosmológica, foi originalmente introduzida por Einstein como uma tentativa de produzir um modelo de universo estático $(\dot{a}=\ddot{a}=0)$ e de curvatura positiva.

As equações (1.20) e (1.21) contém ainda a conservação local de energia:

$$
\dot{\rho}+3 \frac{\dot{a}}{a}(\rho+p)=0
$$

lembrando que $\rho=\sum_{i} \rho_{i}$ é soma das densidades individuais dos diferentes tipos de fluidos que compõem o modelo assim como $p=\Sigma_{i} p_{i}$ representa a pressão total do sistema; portanto, através das equações de estado inerentes às componentes que caracterizam um modelo de universo, obtemos um panorama completo sobre a evolução do fator de escala.

A maior parte dos fluidos de interesse cosmológico pode ser caraterizada por uma equação de estado com a forma 


$$
p_{i}=\omega_{i} \rho_{i}
$$

em que $\omega_{i}$ é constante de estado de um certo fluido identificado pelo índice " $i$ ". Uma vez que não haja conversão entre tipos de fluidos diferentes, a equação (1.22) pode ser facilmente integrada com o auxílio de (1.23), descrevendo a evolução de um dado tipo de fluido como

$$
\rho_{i}=\rho_{i 0}\left(\frac{a_{0}}{a}\right)^{3\left(1+\omega_{i}\right)}
$$

A matéria não relativística (também chamada de poeira) é caracterizada por ser um fluido de pressão nula. A evolução da densidade deste tipo de fluido é portanto

$$
\rho=\rho_{0}\left(\frac{a_{0}}{a}\right)^{3}
$$

A constante de estado para a matéria relativística (como a radiação, por exemplo ) é $\omega_{r}=\frac{1}{3}$ nos conduzindo à solução

$$
\rho_{r}=\rho_{r 0}\left(\frac{a_{0}}{a}\right)^{4}
$$

Para a densidade de energia do vácuo temos que $\omega_{\Lambda}=-1$, implicando em

$$
\rho_{\Lambda}=\rho_{\Lambda 0}=\text { constante. }
$$

\subsubsection{Parâmetros Cosmológicos Básicos}

É usual em cosmologia trabalharmos com alguns parâmetros fundamentais que, através das observações, caracterizam e distinguem diferentes modelos cosmológicos. Entre os principais temos o parâmetro de Hubble (subseção 1.2.2), além dos parâmetros de densidade e o parâmetro de desaceleração, conforme serão discutidos abaixo.

A densidade crítica do universo $\rho_{c}$ é definida como a densidade total em que o universo é plano, ou seja, tomando-se $k=0$. Assumindo-se os valores atuais das grandezas na primeira das equações em (1.20), temos o valor atual da densidade crítica:

$$
\rho_{c 0} \equiv \frac{3 H_{0}^{2}}{8 \pi G}=1.878 \cdot 10^{-29} h^{2} \frac{g}{\mathrm{~cm}^{3}}
$$


Através da grandeza acima, definimos o parâmetro de densidade de uma dada componente do universo com densidade $\rho_{i}(t)$ como sendo $\Omega_{i}=\rho_{i} / \rho_{c}$. Esta grandeza tem a seguinte propriedade (1.20):

$$
1=\Omega_{k}+\sum_{i} \Omega_{i}
$$

onde a somatória é feita sobre todas as componentes de densidade incluídas no modelo de universo. Portanto, vemos que o sinal do parâmetro de curvatura é determinado pelo conteúdo material do universo:

$$
\begin{aligned}
k>0 \quad \Leftrightarrow \quad \sum_{i} \Omega_{i}>1 \Rightarrow & \text { Universo Fechado } \\
k=0 \quad \Leftrightarrow \quad \sum_{i} \Omega_{i}=1 \Rightarrow & \text { Universo Plano } \\
k<0 \quad \Leftrightarrow \quad \sum_{i} \Omega_{i}<1 \Rightarrow & \text { Universo Aberto }
\end{aligned}
$$

Um exemplo importante é dado ao supormos um universo de curvatura arbitrária formado por vácuo, matéria não relativística e relativística. Neste caso, a relação entre o parâmetro de Hubble e os parâmetros de densidade podem ser escritos explicitamente, com auxílio das equações (1.25), (1.26) e (1.27) e (1.20):

$$
\frac{H^{2}}{H_{0}^{2}}=\Omega_{m} x^{-3}+\Omega_{r} x^{-4}+\Omega_{\Lambda}+\Omega_{k} x^{-2},
$$

em que $\Omega_{m}, \Omega_{r}, \Omega_{\Lambda}$ são, respectivamente, os parâmetros de densidade da matéria nãorelativística, radiação e vácuo, observados atualmente, e $x \equiv a / a_{0}$ (Weinberg, 2008).

Outro importante parâmetro cosmológico que serve de indicador direto para a dinâmica do universo é o parâmetro de desaceleração $q$, definido como

$$
q(t) \equiv-\frac{\ddot{a} a}{\dot{a}^{2}}
$$

O parâmetro de desaceleração hoje, $q_{0}$, é definido como o valor atual de $q(t)$. Se $q_{0}>0$, então $\ddot{a}<0$ e o universo se expandirá desaceleradamente. Se $q<0$, temos $\ddot{a}>0$ e, portanto, a expansão será acelerada. 


\subsubsection{Redshift}

O parâmetro de avermelhamento z (redshift ) é uma grandeza relativística de fundamental importância por ser um observável que possui ligação direta ao fator de escala em um dado instante da evolução do universo.

Consideremos um pico de uma onda eletromagnética emitida em um dado instante $t_{e}$ por um objeto qualquer situado nas coordenadas espaciais $\left(r_{1}, \phi, \theta\right)$, tendo como origem deste sistema de coordenadas a própria Terra. Esta onda eletromagnética viajará por coordenadas $\theta$ e $\phi$ constantes ao longo de uma geodésica nula de modo que teremos, a partir de (1.17), a relação

$$
\int_{t_{e}}^{t_{0}} \frac{d t}{a(t)}=\int_{0}^{r_{1}} \frac{d r}{\sqrt{1-k r^{2}}}=f\left(r_{1}\right),
$$

onde $t_{0}$ representa o instante em que a onda é observada. Se o próximo pico da dada onda emitida em $r$ deixar o objeto em $t_{e}+\delta t_{e}$, teremos então:

$$
\int_{t_{e}+\delta t_{e}}^{t_{0}+\delta t_{0}} \frac{d t}{a(t)}=f\left(r_{1}\right) .
$$

Como $r_{1}$ é a coordenada comóvel do objeto emissor, seu valor permanecerá constante no tempo e, consequentemente, $f\left(r_{1}\right)$ também será. Observando-se que na escala de tempo de emissão de dois picos seguidos de uma onda eletromagnética $\left(\sim 10^{-14} s\right)$ o fator de escala varia muito pouco, decorre que (Weinberg, 1972; Kolb e Turner, 1990; Weinberg, 2008)

$$
\frac{\delta t_{0}}{a\left(t_{0}\right)}=\frac{\delta t_{e}}{a\left(t_{e}\right)}
$$

Portanto, a relação entre as frequências emitida e observada $\left(\nu_{e}\right.$ e $\nu_{0}$, respectivamente) é

$$
\frac{\nu_{e}}{\nu_{0}}=\frac{a\left(t_{0}\right)}{a\left(t_{e}\right)} \equiv \frac{a_{0}}{a_{e}}
$$

Expressando a relação acima em função do parâmetro de redshift z temos:

$$
z:=\frac{\lambda_{0}-\lambda_{e}}{\lambda_{e}}=\frac{a_{0}}{a_{e}}-1 .
$$

onde $\lambda_{0}$ é a frequência observada, $\lambda_{e}$ a frequência emitida e $\lambda_{0} / \lambda_{e}=\nu_{e} / \nu_{0}$. 


\subsection{Modelos do Big Bang: Base Observacional}

O modelo de Big Bang é sustentado por quatro pilares fundamentais. O primeiro, teórico, se baseia no formalismo da TRG, que tem como consequência as equações de Friedmann, responsáveis pela descrição da dinâmica do universo. Os observacionais são a expansão do universo, descoberta por Hubble nos anos 1930, as abundâncias relativas dos elementos leves produzidos minutos após o Big Bang, explicadas por Gamow nos anos 1940, e a existência de uma radiação de corpo negro permeando o universo, a Radiação Cósmica de Fundo (RCF), observada originalmente por Penzias e Wilson (1965).

As observações sustentam a hipótese de que o universo tenha surgido da expansão de um gás denso e quente. No resfriamento posterior deste gás primordial foram sintetizados os elementos leves e posteriormente surgiram as condições necessárias para o crescimento das estruturas através da amplificação gravitacional de pequenas inomogeneidades iniciais.

A primeira peça constituinte do modelo padrão é a observação de que vivemos em universo em expansão. No contexto da TRG, a expansão do universo é descrita através da variação do fator de escala $a(t)$, com sua dinâmica descrita pelas equações de Friedmann (1.20) e (1.21). A forma como o fator de escala varia é determinada em função dos constituintes do universo.

\subsubsection{Nucleossíntese Primordial}

Logo após o Big Bang o universo era uma sopa cósmica quente e densa, dominada pela radiação. O plasma primordial portanto deveria estar muito mais sujeito a interações do que os constituintes do universo estão atualmente. O resfriamento posterior deste plasma favoreceu o início de processos fora do equilíbrio. O estudo da formação primordial de núcleos parte do cenário descrito acima, tendo como protagonistas as razões entre fótons e bárions e entre nêutrons e prótons.

Os cálculos da síntese de elementos leves a partir dos núcleos de hidrogênio dependem também da relação entre a temperatura, da taxa de expansão e das taxas de reações fracas e nucleares. As reações regidas pela interação fraca determinam a interconversão entre nêutrons e prótons, que por sua vez, determina a quantidade de ${ }^{4} H e$ sintetizada. Já as reações nucleares determinam a relação entre o número de bárions e o número de fótons ( a razão bárion-fóton $\eta$ ) e o número de espécies de neutrinos, $N_{\nu}$, além de regular a produção 
e destruição dos outros elementos leves.

Segundo esta teoria, a síntese primordial dos elementos ocorre entre os instantes $t \approx$ 0.01 s e $t \approx 100 \mathrm{~s}$, quando a temperatura do universo caiu de $T \approx 10 \mathrm{MeV}$ para $T \approx 0.1 \mathrm{MeV}$. Neste intervalo de tempo, houve a formação em quantidades significativas de deutério $\left({ }^{3} H\right)$, hélio-3 $\left({ }^{3} \mathrm{He}\right)$, hélio $\left({ }^{4} \mathrm{He}\right)$ e lítio $\left({ }^{7} \mathrm{Li}\right)$ a partir dos nêutrons e prótons presentes no plasma primordial. Com a queda da temperatura, a pressão sobre os constituintes bariônicos não era mais suficiente para produzir a fusão nuclear no plasma primordial, cessando a síntese dos elementos leves. Portanto, as frações dos elementos leves observadas hoje no universo devem ser aproximadamente iguais àquelas do universo logo após a nucleossíntese.

Segundo as previsões da nucleossíntese, a parte bariônica do universo é constituída por cerca de $75 \%$ de hidrogênio $\left({ }^{1} H\right)$, $25 \%$ hélio $\left({ }^{4} \mathrm{He}\right)$ e menos que $1 \%$ de outros elementos.

Por outro lado, este cenário não é capaz explicar de explicar as abundâncias dos elementos mais pesados. A ausência de núcleos estáveis com massa 5 e 8 não permite que as fusões necessárias à formação de elementos mais pesados continuem significantemente. Este problema foi posteriormente explicado por Hoyle (Burbidge et al., 1957), propondo que elementos mais pesados fossem criados por processos nucleares no interior das estrelas, na chamada nucleossíntese estelar.

As estimativas de abundâncias de elementos constituem um importante teste para os modelos cosmológicos. Steigman (2006) mostrou que é possível relacionar de forma simples e precisa as abundâncias a alguns observáveis físicos, como por exemplo, a razão fótonbárion, $\eta$. Se baseando nesta abordagem, a análise dos dados do Wilkinson Microwave Anisotropy Probe(WMAP) permite realizar previsões acerca da nucleossíntese. Um breve resumo destas previsões e de valores observados é apresentado na tabela 1.1.

Dentre as abundâncias primordiais, o melhor bariômetro é o deutério devido à sua sensibilidade ao valor de $\eta$. Baseado em medidas de linhas de absorção de quasares, Kirkman et al. (2003) estimou a abundância primordial de deutério como $10^{5} y_{D}^{F I T}=2.78_{-0.38}^{+0.44}$ para o modelo $\Lambda \mathrm{CDM}$ plano $\Sigma m_{\nu}=0.58 \mathrm{eV}$ (95\% c.e.) (Komatsu et al., 2010)

As análises das abundâncias primordiais dependem sempre da razão fóton-bárion, $\eta=$ $[2-6] \times 10^{-10}$ e do número de espécies de neutrinos relativísticos, $N_{\nu}<3.9$ (Steigman, 2000; Copi et al., 1995). Com o auxílio da temperatura da RCF, estimada em $T_{0}=2.725 \pm 0.001$ K (Smoot et al., 1992), o conteúdo bariônico do universo pode ser estimado como 


\begin{tabular}{||c|c|c||}
\hline \hline Parâmetro & Abundância baseada na RCF & Valores Observados \\
\hline \hline $10^{5} y_{D}^{F I T}$ & $2.57_{-0.13}^{+0.17}$ & $1.6-4.0$ \\
\hline $10^{5} y_{3}$ & $1.05 \pm 0.03 \pm 0.03($ sist $)$ & $<1.1 \pm 0.2$ \\
\hline$Y_{P}$ & $0.24819_{-0.00040}^{+0.0009} \pm 0.0006($ sist $)$ & $0.232-0.258$ \\
\hline$[L i]_{P}$ & $2.64 \pm 0.03$ & $2.2-2.4$ \\
\hline \hline
\end{tabular}

Tabela 1.1 - Abundância dos elementos produzidos na Nucleossíntese Primordial. $y_{D}^{F I T}$ representa a abundância do deutério, $y_{3}$ é a abundância do hélio-3, $Y_{p}$ é a abundância do hélio e $[L i]_{P}$ representa uma função da abundância do lítio-7, $\left.[L i]_{P}=12+\log _{10}(L i / H)\right)$. Valores calculados utilizando a razão fóton-bárion $\eta_{10}=6.116_{-0.249}^{+0.197}$

$$
\Omega_{b} h^{2}=0.019 \pm 0.01
$$

ou ainda, utilizando $h=0.72$ (Freedman et al., 2001)

$$
\Omega_{b}=0.045 \pm 0.005 \text {. }
$$

A teoria da nucleossíntese também se mostra em acordo com as estimativas acerca de $N_{\nu}$ obtidas em aceleradores (Turner e White, 1997). Além de fornecer um limite superior para a massa de neutrinos (Larson et al., 2010), seus resultados podem ser usados para vincular outros parâmetros cosmológicos.

\subsubsection{Radiação Cósmica de Fundo}

Nos estudos pioneiros da síntese dos elementos leves, Gamow e colaboradores chegaram à conclusão de que o universo deveria ser permeado por uma radiação com temperatura estimada em aproximadamente $10 K$, valor que fora recalculado por Alpher e Herman para $T_{\gamma 0}=5 K$, em uma análise mais detalhada em 1950. A despeito de tal previsão teórica, restavam dúvidas sobre a capacidade de sobrevivência desta radiação, uma relíquia do Big Bang.

A questão voltou a ser estudada por Dicke, Peebles, Roll e Wikinson em 1965. Estes não apenas tentaram re-estimar $T_{\gamma 0}$ como também levaram a sério a idéia de medir esta radiação de corpo negro, através de um experimento preparado por Roll e Wilkinson. Contudo, antes que o experimento se completasse, Roll e Wilkinson tiveram contato com 
uma observação realizada por Penzias e Wilson, dois engenheiros dos laboratorios Bell, que mediram "um excesso de temperatura de antena", um fraco sinal de fundo no comprimento de onda de $7.35 \mathrm{~cm}$, o equivalente à temperatura de antena de $3.5 \pm 1 K$ (Penzias e Wilson, 1965). Enquanto a distribuição angular da radiação observada tornava impossível associála a qualquer fonte local de rádio ou a algum ruído proveniente de emissões atmosféricas, sua intensidade se mostrava muitas vezes maior que o qualquer erro sistemático possível, levando à conclusão de que esta se tratava da radiação proveniente do Big Bang, a Radiação Cósmica de Fundo (RCF). Os artigos de Penzias e Wilson (1965) e de Dicke et al. (1965) foram publicados juntos, em que o segundo explicou a importância da medida realizada (Weinberg, 1972).

A principal característica da RCF é que ela é uma radiação com espectro de corpo negro à temperatura de $T_{0}=2.725 \pm 0.001 K$, altamente isotrópica (Smoot et al., 1992). Esta isotropia sugere que a RCF é um mar de radiação permeando todo o universo uniformemente e, portanto, um observador comóvel situado em outra galáxia veria a mesma intensidade de radiação isotropicamente, em perfeito acordo com princípio cosmológico.

A natureza de sua observação está diretamente ligada com o limite no passado que podemos observar. Vimos na seção 1.2.3 que a densidade de matéria tem dependência com o fator de escala cósmico de $\rho_{M} \propto a^{-3}$, enquanto a densidade de radiação varia com $\rho_{R} \propto a^{-4}$. Retrocedendo as equações, é esperado que haja um momento em que a densidade de radiação domine a densidade de matéria, assim como se espera que o universo estivesse muito mais aquecido do que observamos hoje.

Em um universo quente e denso, elétrons e prótons se mantinham interagindo fortemente com os fótons (via espalhamento Compton) não permitindo a formação de átomos neutros e de estruturas bariônicas. O resfriamento decorrente da expansão fez com que os fótons diminuíssem abruptamente as suas interações com elétrons levando à recombinação dos elétrons livres. Quando o universo tem a temperatura de cerca de $3000 \mathrm{~K}$, correspondente à idade de aproximadamente $10^{7}$ anos, os fótons já não possuíam energia suficiente para sustentar o processo de ionização dos átomos de hidrogênio. Nesse momento, houve a neutralização dos átomos e os fótons passaram a viajar livremente pelo universo, praticamente sem interagir com a matéria, período denominado como Recombinação. Em relação ao redshift, este evento ocorre em $z \approx 1100$, determinando a região a partir da qual os 
fótons passam a se propagar livremente, a superfície de último de espalhamento.

Antes da recombinação, fótons e elétrons se mantinham fortemente acoplados, formando o chamado fluido fóton-bárion. Ao se desacoplar da matéria, o campo de radiação mantém congelado em seu espectro as flutuações do fluido fóton-bárion, carregando as informações relacionadas às inomogeneidades daquele fluido. Desde então, RCF passou então a se propagar praticamente sem interagir e, portanto, mantendo estas anisotropias primárias gravadas em seu espectro mas se resfriando devido à expansão do universo.

Os mapas do céu em microondas foram originalmente obtidos pelo satélite COBE (Cosmic Background Explorer) (Smoot et al., 1992). Mais recentemente, o satélite WMAP da NASA, vem mapeando as anisotropias da RCF com precisão muito maior. Estas anisotropias são geradas pelas perturbações do potencial gravitacional na superfície de último espalhamento. Desta forma, as posições e amplitude dos picos que aparecem no espectro de potência das anisotropias (ver figura 1.1) têm importante ligação com os parâmetros cosmológicos básicos.

O primeiro pico acústico do espectro está relacionado com o tamanho do horizonte na época da recombinação, e é bastante sensível à geometria do universo. A excelente concordância entre sua posição observada $(l \approx 220)$ com as previsões teóricas é um dos grandes triunfos do modelo padrão. O segundo pico tem sua amplitude relacionada à quantidade à matéria bariônica (incluindo a parte "escura" da matéria bariônica), fornecendo vínculos para $\Omega_{b}$, enquanto o terceiro pico é mais sensível à densidade física de matéria escura $(\mathrm{Hu}$ e Dodelson, 2002).

Embora a estrutura dos primeiros picos detectados já sejam grande fonte de informação, nas posições e amplitudes dos picos adjacentes estão guardadas uma rica física que só poderá ser decifrada quando as medidas em pequenas escalas se tornarem mais precisas.

É importante destacar que os dados do WMAP indicam com grande precisão que o universo seja (aproximadamente) plano, como previsto pela teoria da inflação. A radiação cósmica de fundo é uma das relíquias mais antigas do universo e que se mantém praticamente intacta desde o desacoplamento. Certamente é uma das mais precisas e importantes fontes de informação sobre o universo primordial e atual. 


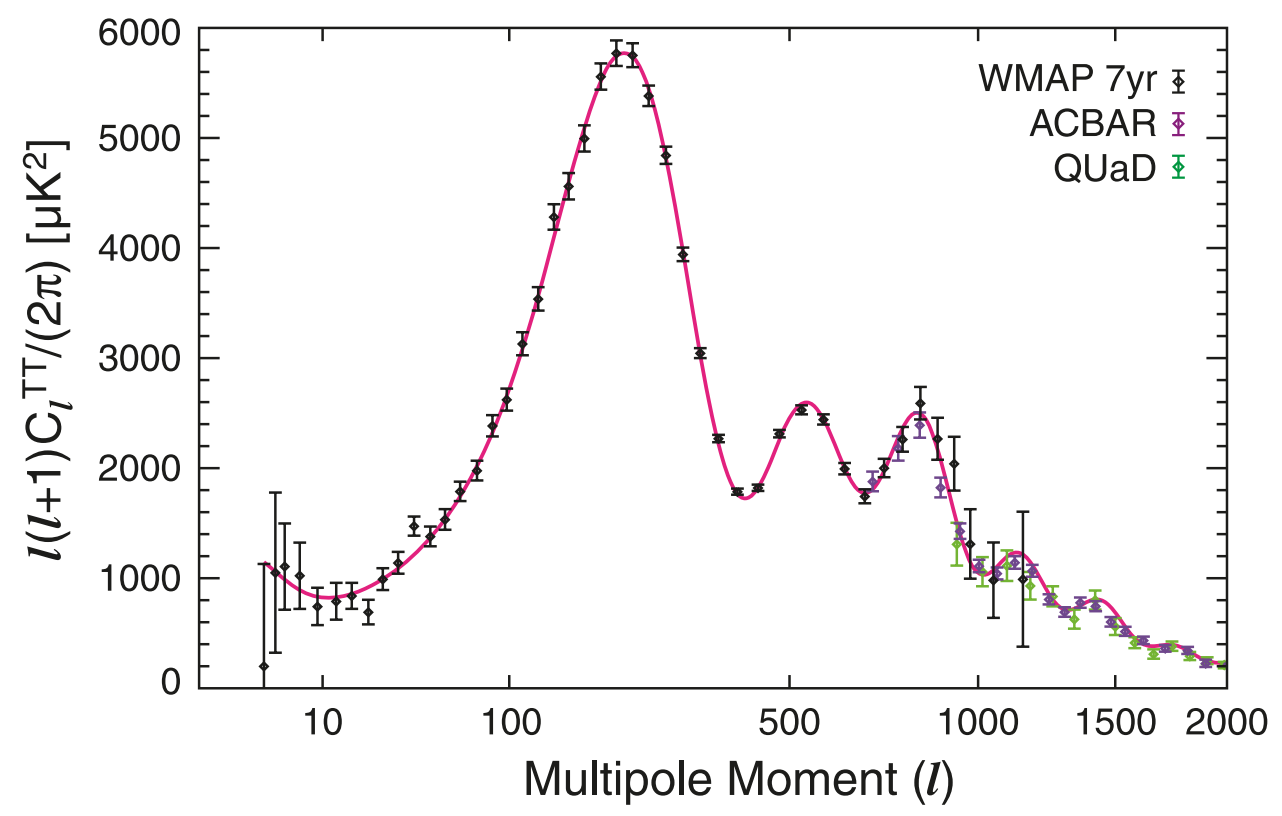

Figura 1.1: Anisotropias de temperatura da Radiação Cósmica de Fundo. Espectro angular de potências obtido pelo WMAP e outros experimentos em pequenas escalas (Komatsu et al., 2010).

\subsection{Modelo de Concordância Cósmica}

\subsubsection{Matéria Escura}

Quase toda a informação extra-galática a que temos acesso direto nos é transmitida através da radiação eletromagnética. Ainda assim, o modelo padrão prevê que a maior parte do conteúdo material do universo não interage por este meio. A chamada matéria escura, apesar de não interagir com os fótons (daí a denominação "escura"), imprime seus efeitos em todas as escalas do universo, seja por meio de sua interferência na forma das curvas de rotação das galáxias nas velocidades orbitais em aglomerados, em distribuições observadas em gases de aglomerados, por lentes gravitacionais, entre outros, sempre detectada por seus efeitos gravitacionais.

Mecanismos baseados em potenciais newtonianos revelam que a massa esperada para galáxias e aglomerados de galáxias é muito superior à massa atribuída à relação MassaLuminosidade para os dados sistemas. As primeiras indicações da existência de matéria escura remetem aos trabalhos de Zwicky (1933) e Smith (1936) onde estes, utilizando o teorema do virial, tentaram inferir a massa dos aglomerados de Coma e Virgo concluindo que a massa total estimada não poderia ser atribuída somente à matéria visível. 
Os estudos do comportamento das curvas de rotação de galáxias também fornecem evidências da existência de matéria escura. As curvas de rotação são traçadas através das medições da velocidade circular orbital em função da distância radial ao centro galático, utilizando-se estrelas e nuvens de hidrogênio neutro (HI) como partículas de teste. Quando Rubin e Ford Jr. (1970) mediram curvas de rotação para a galáxia M31, era esperado que, para pequenos raios, a curva de rotação tivesse o comportamento da curva de um corpo rígido e, uma vez que pressupunha-se que a maior parte da massa da galáxia estivesse contida na região em que a matéria luminosa se concentra, o comportamento da curva deveria ser kepleriano em médios e grandes raios.

Enquanto que para as pequenas distâncias do centro galático o comportamento esperado foi confirmado, surpreendentemente as partes externas das curvas de rotação não seguiam a lei kepleriana. Na verdade, no regime de médios e grandes raios, a curva de rotação permanece aproximadamente constante. Este comportamento já foi observado em diversas outras galáxias, indicando a existência de um componente massivo, dominante em relação à matéria luminosa nas partes mais externas formando um halo escuro (figura 1.2).

Em aglomerados de galáxias, a contribuição da matéria em forma de gás interestelar excede a contribuição das estrelas em pelo menos uma ordem de magnitude, de modo que a massa bariônica total é bem representada pela massa do gás (Forman e Jones, 1982; White et al., 1993). Através das medidas de 19 aglomerados, White e Fabian (1995) concluíram que a razão entre a massa do gás e a massa dinâmica do aglomerado é:

$$
\frac{M_{\text {gás }}}{M_{\text {din }}}=0.056 h^{-2 / 3}
$$

Tomando a razão fóton-bárion em unidades de $10^{-10}$ como $\eta_{10}=3-5$ e $h=70$, o parâmetro de densidade fica como:

$$
\Omega_{m}=0.2-0.4
$$

A necessidade de uma componente adicional não-bariônica se torna, portanto, evidente quando comparamos as estimativas mostradas com o parâmetro de densidade dos bárions restringido pela abundância primordial de elementos $\left(\Omega_{b}=0.045 \pm 0.005\right)$.

Recentemente, observações do aglomerado 1E0657-558, conhecido como "Bullet Cluster", forneceram uma prova empírica direta da existência de matéria escura. Este objeto 


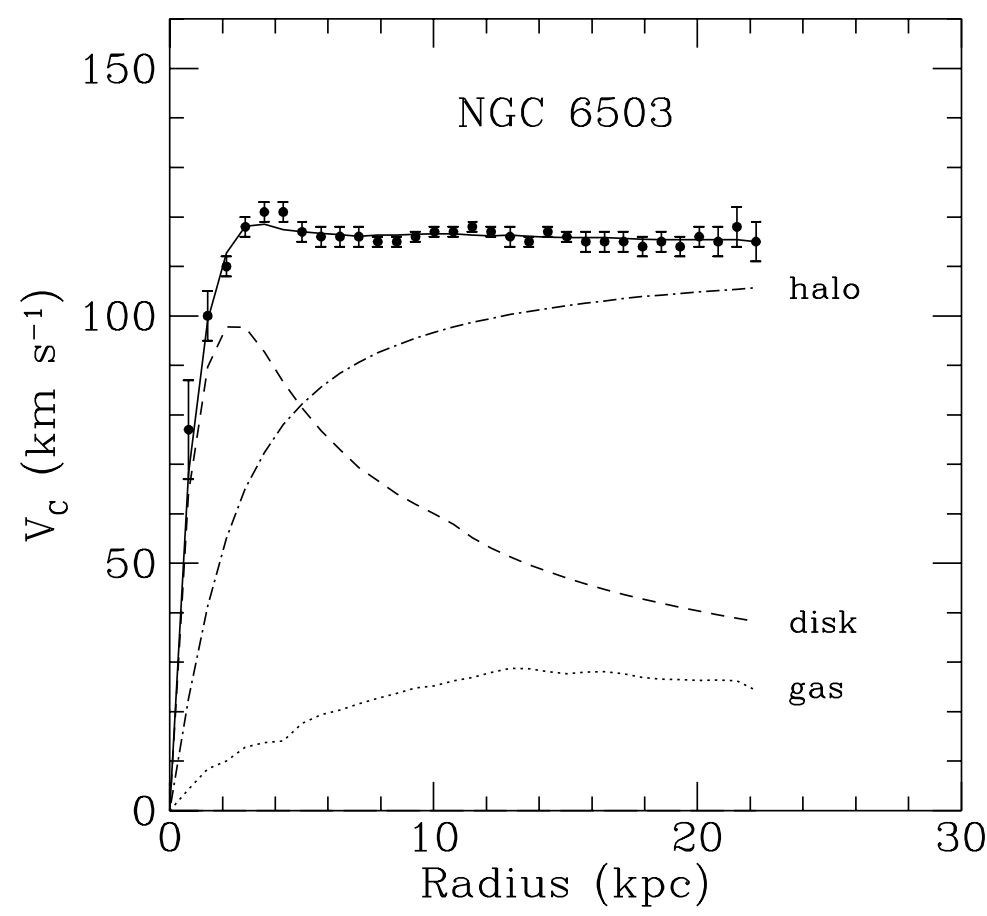

Figura 1.2: Curva de rotação da galáxia espiral NGC6503. Os pontos representam medidas da velocidade orbital circular em função da distância ao centro da galáxia, enquanto as linhas representam as contribuições para a velocidade rotacional devido ao disco, gás e halo escuro. O comportamento não kepleriano da curva para altos raios é explicado através da presença do halo escuro. Figura retirada de (Begeman et al., 1991).

é resultado do processo de fusão de dois aglomerados ainda em andamento (Tucker et al., 1998). Como resultado temos um sistema formado por quatro componentes distinguíveis: uma componente estelar e uma de gás emitindo em raios - X para cada um dos aglomerados progenitores, componentes que hoje se encontram espacialmente segregadas. Esta separação entre componentes é observada pois, durante o processo de colisão, enquanto a parte estelar se comporta como um fluido acolisional, o gás intra-aglomerado sofre a pressão de arraste (ram - pressure) (Clowe et al., 2006).

Os mapas de potencial obtidos através de estudos por lentes gravitacionais fortes e fracas mostraram que os centros de massa total de cada um dos dois sistemas progenitores em interação não acompanham os centros de massa de suas componentes na forma de plasma (figura 1.3). Como a maior parte da massa bariônica do aglomerado corresponde ao gás de plasma, então a maior parte da matéria está contida em uma forma não visível. 
Estudos de outros dois sistemas de mesmas características encontrados posteriormente se mostraram em acordo com esta conclusão (Bradač et al., 2008).
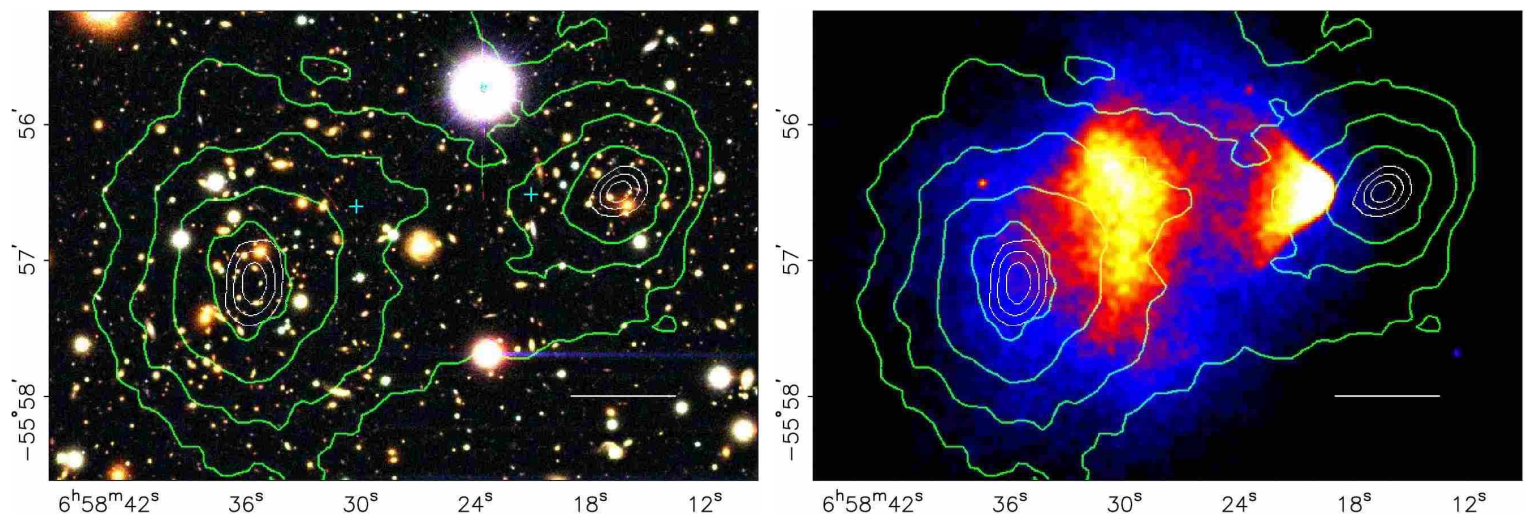

Figura 1.3: Imagens do aglomerado em fusão 1E0657-558, a) no visual e b) em exposição de 500 ks em raios-X . Os contornos verdes mostram a função $\kappa$, proporcional à densidade superficial de massa, onde o contorno mais externo representa $\kappa=0.16$, e os contornos internos foram calculados subtraindo-se $\Delta \kappa=0.07$. A barra branca no canto inferior tem $500 \mathrm{kpc}$ à distância do aglomerado. Figura retirada de (Clowe et al., 2006).

Além do que foi mostrado aqui, temos outras importantes fontes de evidência da existência da matéria escura como (i) as funções de autocorrelação entre masas de galáxias e massas de halos, (ii) a temperatura e a polarização das anisotropias da radiação cósmica de fundo, (iii) estudos das estruturas de grandes escalas através das oscilações acústicas dos bárions (BAO), (iv) simulações das estruturas de grandes escalas, (v) ajustes cosmológicos dos dados de RCF, BAO e SNe Ia, entre outros (Roos, 2010).

É importante mencionar também que, se admitíssemos que o crescimento de perturbações do universo fosse puramente bariônico, seria necessário que as perturbações observadas na $\mathrm{RCF}$ fossem $\approx 100$ vezes maior do que o observado $\left(\delta T / T \approx 10^{-5}\right.$ ) (Komatsu et al., 2010). A teoria de formação de estruturas nos leva a concluir que é necessário uma componente de matéria escura para que haja a formação de galáxias. Em síntese, isto é explicado pelo fato de que a matéria bariônica se mantém acoplada à radiação até o momento da recombinação e, portanto, não pode desenvolver estruturas até este dado momento. Já a formação de estruturas na matéria não-bariônica pode começar anteriormente, a partir da equipartição matéria-radiação, tendo mais tempo para desenvolver estas perturbações.

As evidências observacionais apontam que esta componente extra de matéria deve ser 
fria, i.e., não-relativística (Peacock, 1999). Enquanto a matéria escura quente (relativística) apaga as flutuações de pequena escala na época de formação de estruturas, a matéria escura fria (Cold Dark Matter- CDM) consegue se acumular em escalas pequenas, formando as estruturas no esquema bottom-up (das menores para as maiores). Esta última descrição está de acordo com o cenário hierárquico, o que melhor descreve as observações.

Por mais que a natureza da matéria escura ainda não tenha sido elucidada, devemos destacar que a nucleossíntese primordial e as teorias de formação de estruturas associadas às observações acerca da RCF excluem a possibilidade de que a matéria escura seja formada por matéria bariônica, como restos de estrelas massivas e buracos negros. Portanto, a matéria escura é componente essencial para qualquer modelo cosmológico capaz de explicar o universo observado.

\subsubsection{Distâncias Cosmológicas}

Nas medidas de distância cosmológicas, devemos levar em conta os efeitos impostos pela curvatura e pela própria expansão do universo. Tais distâncias não são invariantes sendo, na verdade, definidas em função do observável. A relação entre as medidas é bem definida e, em baixos redshifts, convergem entre si. Especificamente, discutiremos aqui duas medidas de distância de importância fundamental para os testes cosmológicos: a distância de luminosidade e a distância de diâmetro angular.

Primeiramente, calcularemos a coordenada comóvel radial através da métrica (1.17). Uma vez que a luz sempre viaja por geodésicas nulas $\left(d s^{2}=0\right)$, temos:

$$
\int_{0}^{r} \frac{d r}{\sqrt{1-k r^{2}}}=\int_{t}^{t_{0}} \frac{d t}{a(t)}=\text { constante }
$$

para dois observadores comóveis localizados na coordenada $r$ e na origem, respectivamente.

A distância de luminosidade estabelece a relação entre a luminosidade aparente $l$ e a luminosidade absoluta $L$ de uma dada fonte. Imaginemos um telescópio com área de abertura $A$ recebendo a luz proveniente de uma fonte no redshift $z$ com luminosidade absoluta L. Em um espaço representado por uma métrica diagonal, a área total compreendida pela frente de onda esférica em um dado instante $t$ é calculada a partir do elemento de linha, no nosso caso, a equação (1.17): 


$$
S=\int_{0}^{2 \pi} \int_{0}^{\pi} \sqrt{g_{22}} \sqrt{g_{33}} d \theta d \phi=4 \pi a(t)^{2} r^{2},
$$

sendo $r$ a coordenada comóvel do objeto em questão. Desta forma, a fração da luminosidade captada pelo telescópio hipotético é

$$
\frac{A}{4 \pi a(t) r^{2}}
$$

Em nossa dedução, ainda será necessário considerar mais dois efeitos decorrentes da expansão cosmológica. Seja $t_{1}$ o tempo de emissão da fonte e $t_{0}$ o instante em que o fóton emitido chega ao observador. Uma vez que, devido ao redshift, cada fóton emitido tem sua frequência alterada, a energia $h \nu_{1}$ original do fóton será modificada por $h \nu_{0}=h \nu_{1} / a\left(t_{1}\right)=$ $h \nu_{1}(1+z)$ (1.35). A taxa com que os fótons chegam ao observador também é modificada pela relação $\delta t_{0}=\delta t_{1} a\left(t_{1}\right)=\delta t_{1} /(1+z)$. Portanto, a potência $P$ captada em $t_{0}$ é

$$
P=L\left(\frac{a^{2}\left(t_{1}\right)}{a^{2}\left(t_{0}\right)}\right)\left(\frac{A}{4 \pi a^{2}\left(t_{0}\right) r^{2}}\right) .
$$

A luminosidade aparente $l$ é, por sua vez, (Weinberg, 1972)

$$
l \equiv \frac{P}{A}=\frac{1}{4 \pi a^{4}\left(t_{0}\right)(1+z)^{2} r^{2}} .
$$

Em analogia com a expressão de $l$ encontrada para o espaço euclideano, $l=L / 4 \pi d^{2}$, podemos deduzir a forma da distância de luminosidade $d_{L}$ em cosmologias FRW:

$$
d_{L} \equiv a\left(t_{0}\right)(1+z) r
$$

com $r$ calculável através da expressão (1.41).

Expandindo a distância de luminosidade em função de valores atuais, temos:

$$
d_{L}=\frac{1}{H_{0}}\left[z-\frac{1}{2}\left(1+q_{0}\right) z^{2}+\ldots\right]
$$

A existência de barras padrão, objetos astrofísicos de dimensões calculáveis ou previamente conhecidas, nos permite definir a chamada distância de diâmetro angular $d_{A}$, também em analogia com a geometria euclideana, através da relação

$$
\theta=\frac{s}{d_{A}}
$$


sendo $\theta \ll 1$ o ângulo compreendido pela fonte de luz observada, esta com diâmetro próprio (conhecido) $s$ e situado à distância $d_{A}$. Considerando que o objeto emissor esteja situado na coordenada $r$ emitindo em um instante $t_{1}$, enquanto é observado no instante $t_{0}$, temos que, através da integração da expressão (1.17),

$$
s=a\left(t_{1}\right) r \theta \text {. }
$$

Logo, de acordo com (1.48), a distância de diâmetro angular do objeto é

$$
d_{A}=a\left(t_{1}\right) r
$$

Analisando as equações (1.46) e (1.50), a relação entre os dois tipos de distância mostrados acima é

$$
\frac{d_{L}}{d_{A}}=(1+z)^{2}
$$

onde, em baixos redshifts, as distâncias coincidem como esperado, uma vez que estamos abaixo do regime cosmológico.

\subsubsection{Supernovas e Aceleração do Universo}

Uma das características mais intrigantes do universo foi revelada em 1998, através das observações de Supernovas do Tipo Ia (SN Ia): a expansão acelerada do universo. Até os anos 1990, acreditava-se que o universo era constituído apenas por matéria e radiação, descrito pelo modelo de Einstein-de Sitter. As melhorias na compreensão sobre o comportamento das curvas de luz de supernovas tipo Ia permitiram que estas fossem utilizáveis como velas-padrão, principalmente nas medidas em altos redshifts $(z>0.1)$ por se tratarem de objetos muito brilhantes (em média, magnitude $m \approx 19.2$, no pico da curva de luz). As comparações entre as observações de SN Ia e previsões teóricas foram realizadas simultaneamente por dois grupos independentes: o Supernova Cosmology Project e o High-z Supernova Search.

O grupo do Supernova Cosmology Project estudou 42 supernovas com redshifts entre 0.18 e 0.83 , juntamente com um subconjunto de supernovas com redshifts menores que 0.1 concluindo que, para um modelo composto de matéria escura e constante cosmológica, 
$\Omega_{\Lambda}=0$ está excluído com confiança estatística de $99 \%$, independentemente da curvatura da seção espacial. Para um modelo plano, o melhor ajuste foi de $\Omega_{M}=0.28$, o que resulta em um parâmetro de desaceleração $q_{0}=-0.58$, indicando que o universo está em uma fase de expansão acelerada. Os resultados obtidos estão apresentados na figura 1.4.

Já o grupo do High-z Supernova Search estudou 16 supernovas com redshifts entre 0.16 e 0.97, incluindo duas supernovas do Supernova Cosmology Project, e 34 supernovas com baixos redshifts, chegando à conclusão de que para um modelo com matéria escura e constante cosmológica temos que $\Omega_{\Lambda}>0$ com um nível de confiança estatística de $99.7 \%$, independente da curvatura da seção espacial. O melhor ajuste para um modelo plano foi de $\Omega_{M}=0.28$. Supondo $\Omega_{M}>0$, eles obtiveram $q_{0}<0$ com $95 \%$ de confiança estatística (figura 1.5).

Na última década, o tamanho das amostras de Supernovas do tipo Ia cresceram enormemente. A última delas, a amostra Constitution (Hicken et al., 2009), contém 397 supernovas, a maioria delas em redshifts $0.25<z<1.8$.

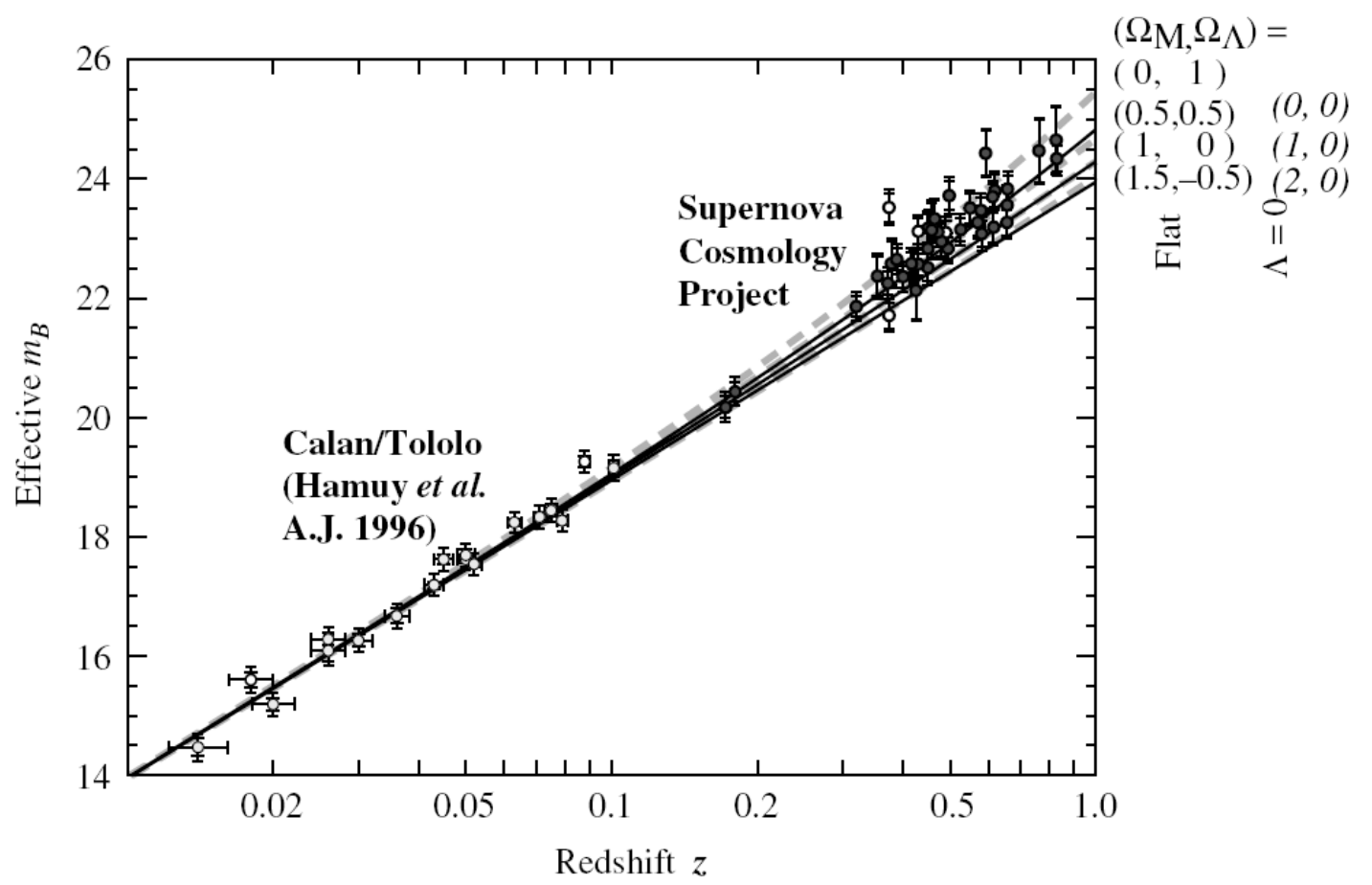

Figura 1.4: Diagrama de Hubble-Sandage contendo as 42 supernovas observadas pelo Supernova Cosmology Project e as 18 supernovas do Cálan-Tololo Supernovae Survey (Hamuy et al., 1996).

Para discutirmos a aceleração no contexto dos modelos de Friedmann, é interessante 


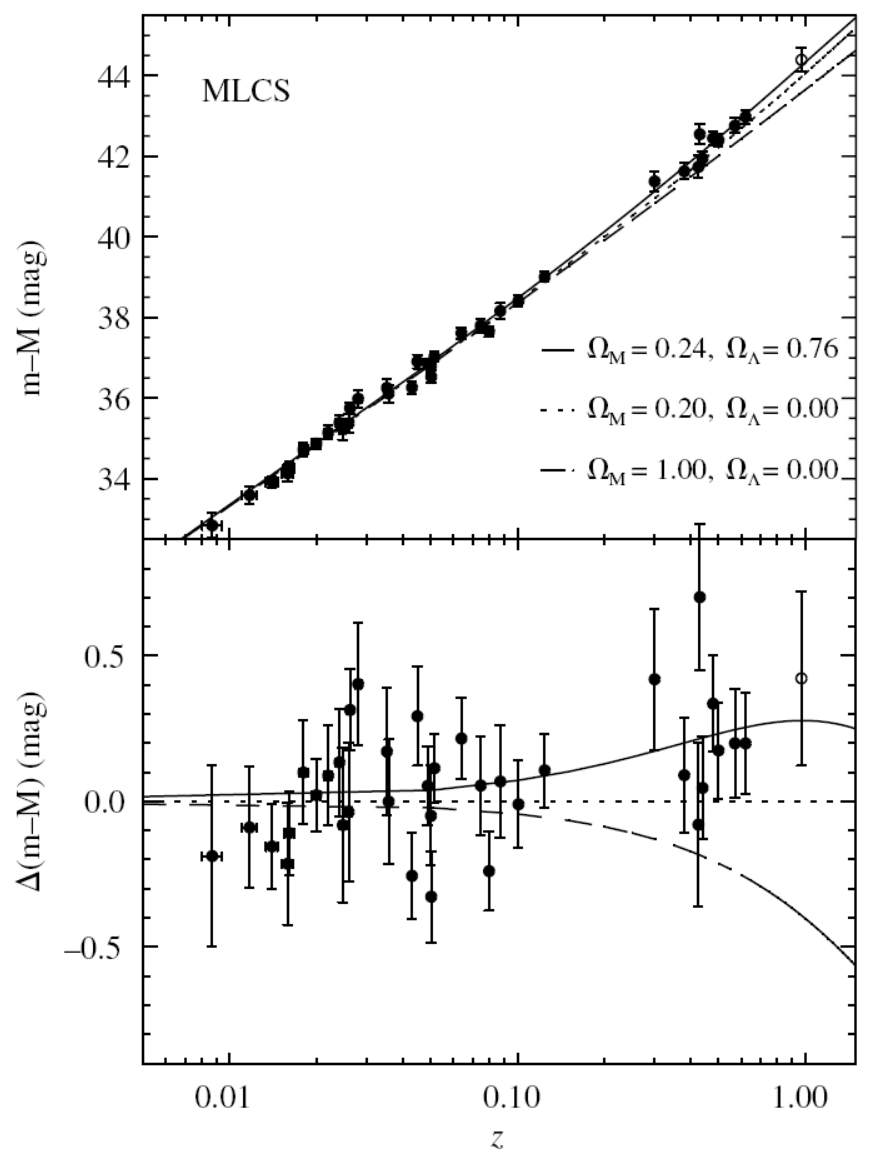

Figura 1.5: Evidência da aceleração do universo obtida com 16 supernovas em altos redshifts, incluindo duas supernovas do Supernova Cosmology Project, e 34 em baixos redshifts observadas pelo High-z Supernova Search. A figura inferior mostra a magnitude residual de um modelo com $\Omega_{M}=0.2$ e $\Omega_{\Lambda}=0$.

observarmos que as equações (1.20) e (1.21) implicam na seguinte expressão:

$$
\frac{\ddot{a}}{a}=-\frac{4 \pi G}{3}\left(\rho+3 p-\frac{\Lambda}{4 \pi G}\right) .
$$

Considerando um fluido usual como fonte de curvatura $(p>0$ e $\rho>0)$, ou pelo menos satisfazendo a chamada condição de energia forte, temos que

$$
\rho+3 p>0
$$

e a aceleração, $\ddot{a}$, será sempre negativa se $\Lambda=0$, com a curva $a(t)$ apresentando concavidade para baixo (Weinberg, 1972). Isso significa também que num tempo finito do passado, o fator de escala assumiu o valor $a=0$, o que implica não apenas em uma di- 
vergência das grandezas físicas, mas também, via Equações de Einstein, na existência de uma singularidade inicial no espaço-tempo (os invariantes de curvatura divergem).

Já modelos com constante cosmológica podem expandir aceleradamente $(\ddot{a}>0)$. Em particular, na era de vácuo e matéria, vemos que a condição $\Lambda>4 \pi G \rho$ garante uma expansão acelerada. Em geral, qualquer fluido com pressão negativa suficientemente grande pode acelerar o universo, como será discutido no próximo capítulo. 


\section{Capítulo 2}

\section{Modelos de Energia Escura}

Além das evidências fornecidas pelos dados de Supernovas do tipo Ia, observações complementares nos levam a inferir a existência da energia escura. Entre elas, podemos citar as anisotropias do espectro de potência da radiação cósmica de fundo (Komatsu et al., 2008), estruturas em grande escala (Cole et al., 2005; Tegmark et al., 2004), observações em raios X de aglomerados de galáxias (Lima et al., 2003), objetos velhos em altos redshifts (Krauss, 1997; Alcaniz e Lima, 1999), tamanho angular de fontes de rádio compactas (Gurvits et al., 1999; Lima e Alcaniz, 2002) e rádio-galáxias (Daly e Guerra, 2002), gammaray bursts (Schaefer, 2007). A figura 2.1 resume a genealogia da energia escura.

Neste capítulo discutiremos os principais candidatos à energia escura. Mostraremos também que existem alternativas onde esta componente cosmológica extra pode ser dispensada, seja por modificações na teoria padrão de gravitação, como propõe as teorias conhecidas como $f(R)$, ou ainda dentro do contexto da Relatividade Geral, através dos modelos com criação de matéria.

\subsection{O Termo $\Lambda$}

Entre os candidados a energia escura a constante cosmológica, $\Lambda$, é o mais simples e mais antigo. Introduzida por Einstein e posteriormente rejeitada pelo mesmo, esta constante tem sido proposta na literatura em diferentes ocasiões, com diferentes interpretações e sempre como uma tentativa de compatibilizar os dados observacionais de cada época.

Em 1917, Einstein inaugurou a era da cosmologia moderna aplicando as idéias da Relatividade Geral ao universo como um todo e introduzindo a constante cosmológica. Para este fim, ele abandonou as suas equações de campo originais em favor de uma proposta con- 


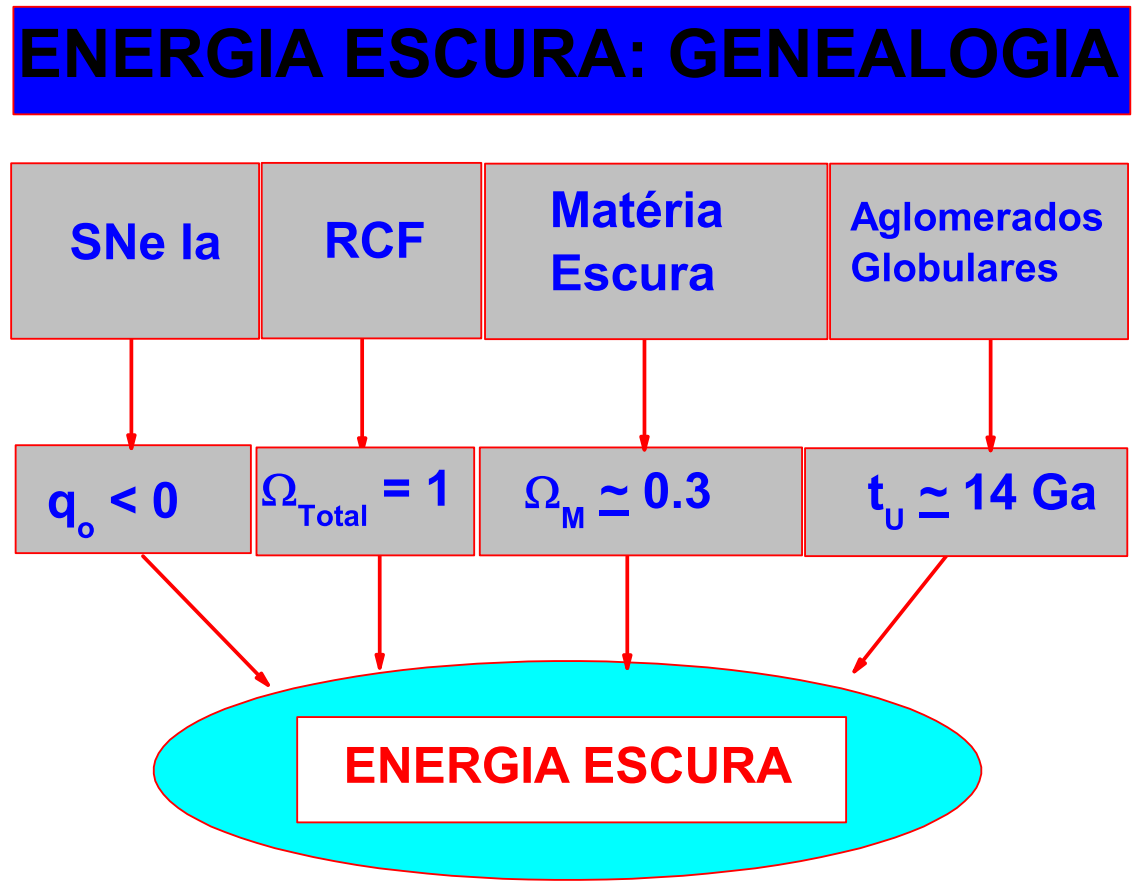

Figura 2.1: Genealogia da energia escura.

tendo um termo constante adicional, que representa um potencial gravitacional constante repulsivo. Na geometria de Schwarzchild, o termo fornece uma pequena força repulsiva aumentando proporcionalmente à distância.

No seu trabalho original, Einstein tinha a intenção de obter um modelo de universo estático. Sem o termo cosmológico, um modelo estático auto-gravitante colapsaria em virtude da ação gravitacional da matéria. Após a descoberta da expansão universal por Hubble, Einstein rejeitou a constante cosmológica alegando suas inconsistências física e estética.

Contudo, muitos autores defendem que teoricamente, a Relatividade é enriquecida pela adição do novo termo, ao mesmo tempo em que ainda mantém as características de uma teoria covariante para a gravitação. De fato, ao adotá-la Einstein deixou de prever a expansão do universo e com o seu descarte restringiu desnecessariamente a generalidade de sua teoria (North, 1965).

O significado da importância da constante cosmológica tem sido matéria de intenso debate na literatura (Lima, 2004; North, 1965; Waga, 1993; Sahni e Starobinsky, 2000). Uma visão histórica das diversas mortes e ressurreições de $\Lambda$ está apresentada no artigo de 
Weinberg (1989), entitulado "O problema da Constante Cosmológica".

A recente descoberta da expansão acelerada do universo provocou uma "ressurreição" da constante cosmológica, que poderia fornecer a repulsão cósmica responsável pela aceleração. O modelo com constante cosmológica, conhecido como $\Lambda C D M$ ou Modelo de Concordância Cósmica, fornece um bom ajuste às principais observações astronômicas. Na tabela 2.1 vemos os vínculos obtidos pelos dados do WMAP (Komatsu et al., 2010) e pela combinação com outros testes para alguns parâmetros cosmológicos no contexto do modelo $\Lambda$ CDM.

Ainda que o modelo $\Lambda C D M$ consiga ajustar bem os principais parâmetros cosmológicos, este não está livre de críticas. Entre suas principais fraquezas, destaca-se o Problema da Constante Cosmológica.

Tabela 2.1 - Modelo $\Lambda$ CDM: Parâmetros cosmológicos do modelo e intervalos de confiança de $68 \%$ (Komatsu et al., 2010).

\begin{tabular}{|c|c|c|c|c|c|}
\hline classe & Parâmetro & WMAP $7(\mathrm{ML})^{\mathrm{a}}$ & $\mathrm{WMAP} 7+\mathrm{BAO}+H_{0}(\mathrm{ML})^{\mathrm{a}}$ & WMAP 7 (Média) b & WMAP $7+\mathrm{BAO}+H_{0}$ (Média) ${ }^{\mathrm{b}}$ \\
\hline \multirow{6}{*}{ Primário } & $100 \Omega_{b} h^{2}$ & 2.270 & 2.246 & $2.258_{-0.056}^{+0.057}$ & $2.260 \pm 0.053$ \\
\hline & $\Omega_{d m} h^{2}$ & 0.1107 & 0.1120 & $0.1109 \pm 0.0056$ & $0.1123 \pm 0.0035$ \\
\hline & $\Omega_{\Lambda}$ & 0.738 & 0.728 & $0.734 \pm 0.029$ & $0.728_{-0.016}^{+0.015}$ \\
\hline & $n_{s}$ & 0.969 & 0.961 & $0.963 \pm 0.014$ & $0.963 \pm 0.012$ \\
\hline & $\tau$ & 0.086 & 0.087 & $0.088 \pm 0.015$ & $0.087 \pm 0.014$ \\
\hline & $\Delta_{R}^{2}\left(k_{0}\right)$ & $2.38 \times 10^{-9}$ & $2.45 \times 10^{-9}$ & $(2.43 \pm 0.11) \times 10^{-9}$ & $\left(2.441_{-0.092}^{+0.088}\right) \times 10^{-9}$ \\
\hline \multirow{7}{*}{ Derivado } & $\sigma_{8}$ & 0.803 & 0.807 & $0.801 \pm 0.030$ & $0.809 \pm 0.024$ \\
\hline & $H_{0}$ & $71.4 \mathrm{~km} / \mathrm{s} / \mathrm{Mpc}$ & $70.2 \mathrm{~km} / \mathrm{s} / \mathrm{Mpc}$ & $71.0 \pm 2.5 \mathrm{~km} / \mathrm{s} / \mathrm{Mpc}$ & $70.4_{-1.4}^{+1.3} \mathrm{~km} / \mathrm{s} / \mathrm{Mpc}$ \\
\hline & $\Omega_{b}$ & 0.0445 & 0.0455 & $0.0449 \pm 0.0028$ & $0.0456 \pm 0.0016$ \\
\hline & $\Omega_{d} m$ & 0.217 & 0.227 & $0.222 \pm 0.026$ & $0.227 \pm 0.014$ \\
\hline & $\Omega_{m} h^{2}$ & 0.1334 & 0.1344 & $0.1334_{-0.0055}^{+0.0056}$ & $0.1349 \pm 0.0036$ \\
\hline & $z_{\text {reion }}$ & 10.3 & 10.5 & $10.5 \pm 1.2$ & $10.4 \pm 1.2$ \\
\hline & $t_{0}$ & 13.71 Ganos & 13.78 Ganos & $13.75 \pm 0.13$ Ganos & $13.75 \pm 0.11$ Ganos \\
\hline
\end{tabular}

a "ML" se refere à estimativa dos parâmetros feita pelo método da Máxima Verossimilhança (Maximum Likelihood).

b "Média"se refere à média da distribuição posterior de cada parâmetro

Em sua interpretação contemporânea, a constante cosmológica está associada a uma densidade de energia

$$
\rho_{\Lambda}=\frac{\Lambda}{8 \pi G}
$$


e a uma pressão

$$
p_{\Lambda}=-\frac{\Lambda}{8 \pi G}
$$

portando, podendo ser interpretada como um fluido perfeito com equação de estado $p=$ $-\rho$.

Em complemento a esta visão, na Teoria Quântica de Campos (TQC) o termo $\Lambda$ está associado às flutuações quânticas do estado do vácuo, comprovadas experimentalmente através do Efeito Casimir (Casimir, 1948; Sparnaay, 1957). Ao contrário da visão clássica, em que o vácuo é entendido como uma região do espaço desprovidade de energia, na TQC o que existe é um estado de vácuo de energia mínima. Em decorrência das relações de incerteza, estes campos flutuam em torno do valor zero enquanto seus valores médios são nulos. Através da invariância de Lorentz do vácuo temos que o tensor de energia-momento deste é $T_{v a c}^{\mu \nu}=\Lambda g^{\mu \nu}$ e, portanto, a equação de estado do vácuo é $p_{v a c}=-\rho_{v a c}$, ou seja, uma equação de estado idêntica a de uma constante cosmológica.

É importante ainda compararmos a estimativa do valor da densidade de energia do vácuo obtida por observações cosmológicas com a previsão teórica.

As observações disponíveis vinculam fortemente que o universo seja plano (Figura 2.2), ou seja, a densidade total de energia deve, ao menos, estar muito próxima da densidade crítica do universo. Podemos então supor que a densidade de energia do vácuo seja dada pela diferença entre a densidade crítica e a densidade de matéria. Uma vez que a densidade de energia escura é dominante hoje, temos (Weinberg, 1989):

$$
\rho_{\Lambda 0} \lesssim \rho_{c 0}=\frac{3 H_{0}^{2}}{8 \pi G} \sim 10^{-29} \mathrm{~g} / \mathrm{cm}^{3}
$$

se tratando portanto de um limite observacional superior para o valor da densidade de energia do vácuo.

Por outro lado, o vácuo de um campo quântico pode ser tratado formalmente por um conjunto infinito de osciladores harmônicos quânticos independentes, cada qual contribuindo com suas próprias oscilações de ponto zero onde cada modo de vibração de um dado campo de frequência $\omega$ contribui com um fator $\frac{1}{2} \hbar \omega$ para a energia total (Zel'dovich, 1968). Desta forma, tomando um campo escalar de massa $m$, a contribuição total de todas as energias de ponto zero de todos os modos normais até um numero de onda $k$ limite 


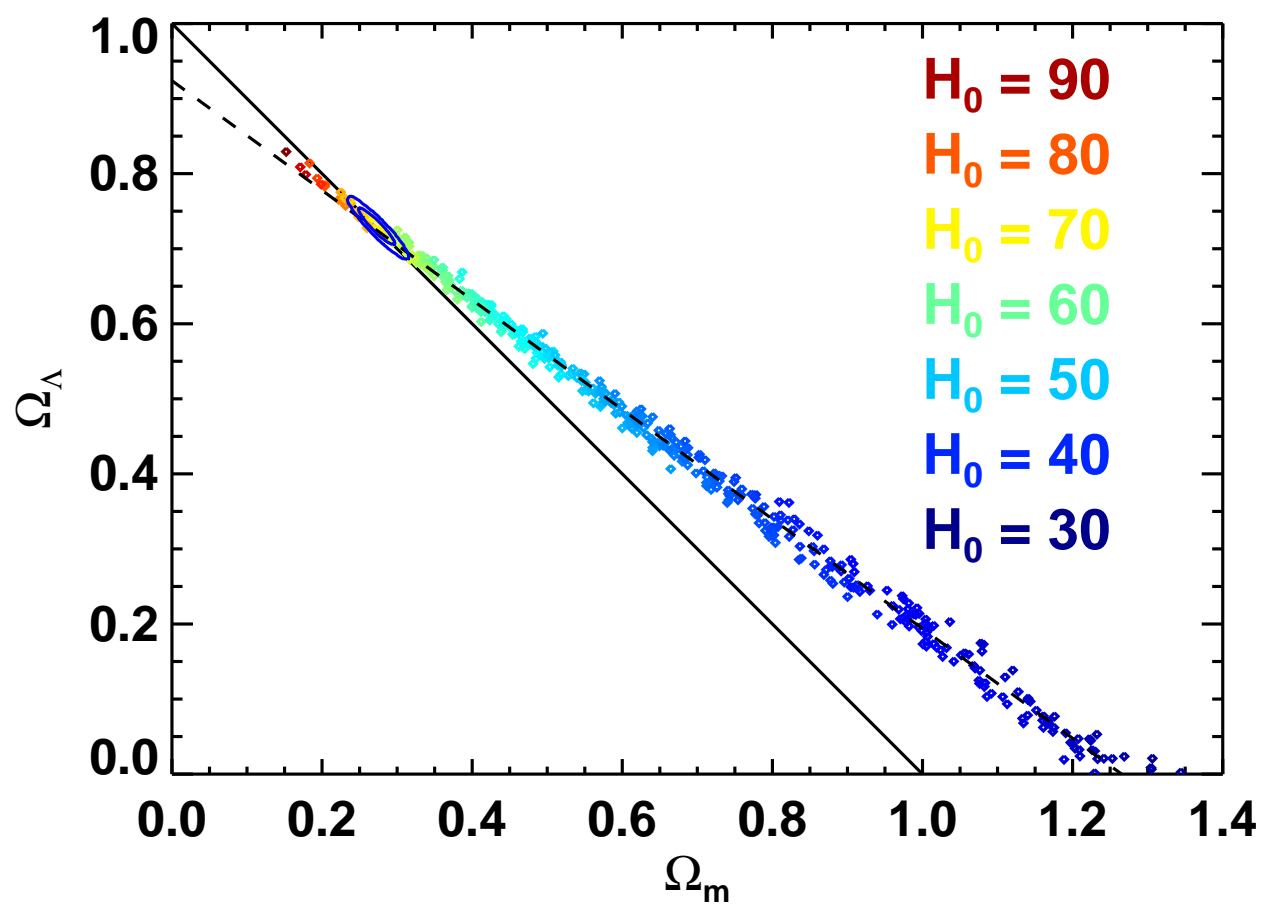

Figura 2.2: Intervalo de modelos cosmológicos não-planos consistentes com os resultados do WMAP. A análise conjunta WMAP $+H_{0}+\mathrm{BAO}$ vincula fortemente um modelo plano de universo (cotornos azuis respectivamente com $68 \%$ e $95 \%$ c.e). Resultado apresentado por Larson et al. (2010).

$(M \gg m)$ resulta em $(\hbar \equiv 1)$ :

$$
<\rho_{v a c}>=\frac{1}{4 \pi^{2}} \int_{0}^{M} k^{3} d k=\frac{M^{4}}{16 \pi^{2}} .
$$

A escala de energia $M$ delimita até que ponto o formalismo da TRG pode ser considerado confiável. Se acreditarmos que este limite é a escala de Planck, o valor da densidade é da ordem de $<\rho>\sim 10^{92} \mathrm{~g} / \mathrm{cm}^{3}$, ou seja, mais de 120 ordens de grandeza superior à estimativa observacional (equação (2.3)). Mesmo assumindo escalas de energia de validade da TRG mais baixas, como por exemplo a escala da Cromodinâmica Quântica, ainda permanecem $\sim 40$ ordens de grandeza de discrepância! Esta enorme diferença entre as previsões observacional e teórica constitui o Problema da Constante Cosmológica (PCC).

Embora ainda não exista uma solução para o PCC baseada em primeiros princípios, algumas alternativas fenomenológicas possíveis têm sido propostas na literatura em que o termo cosmológico efetivo pode variar em função do tempo cósmico. Uma descrição básica 
destes mecanismos, chamados modelos de Decaimento do Vácuo, será dada na seção a seguir.

\subsection{Decaimento do Vácuo $(\Lambda(t))$}

Os modelos $\Lambda(t)$ (Bronstein, 1933; Özer e Taha, 1986; Abdel-Rahman, 1992; Beesham, 1993; Lima e Maia, 1993; Waga, 1993; Arbab e Abdel-Rahman, 1994; Lima e Maia, 1994; Lima, 1996; Lima e Trodden, 1996; Overduin e Cooperstock, 1998) se baseiam na idéia de que a densidade de energia do vácuo pode decair continuamente ao longo da História cósmica. Esta variação em $\Lambda$ estaria diretamente relacionada a interação do vácuo com outras componentes, com a densidade do vácuo diminuindo ao longo da expansão. Esses modelos tentam reconciliar o pequeno valor presentemente observado de $\Lambda$ com o valor absurdamente alto sugerido pelas teorias de campo. Nesse sentido, pode-se dizer que é pequeno porque o universo é muito velho.

Esse modelo é descrito em termos de dois fluídos: um decaimento do vácuo $\left(\rho_{\nu}=\right.$ $\left.\frac{\Lambda(t)}{8 \pi G}, p_{\nu}=-\rho_{\nu}\right)$ mais o produto do vácuo decaindo. Neste caso, as equações de campo de Einstein (ECE) e a lei de conservação de energia são dadas por

$$
\begin{gathered}
8 \pi G \rho+\Lambda(t)=3 \frac{\dot{a}^{2}}{a^{2}}, \\
8 \pi G p-\Lambda(t)=-2 \frac{\ddot{a}}{a}-\frac{\dot{a}^{2}}{a^{2}}, \\
\dot{\rho}+3 H(\rho+p)=-\frac{\dot{\Lambda}(t)}{8 \pi G} .
\end{gathered}
$$

O principal objetivo desse modelo é explicar como a energia conduz a inflação no estágio primitivo, e aceleração do universo, estando relacionada com o pequeno valor corrente de

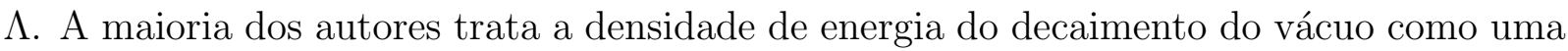
função implícita do tempo, a qual depende do fator de escala $\left(\Lambda \sim a^{-2}\right)$ ou do parâmetro de Hubble $\left(\Lambda \sim H^{2}\right)$, ou ainda uma combinação destes Freese et al. (1987); Calvão et al. (1992); Waga (1993). Uma lista estensiva de fenomenologias da lei de decaimento- $\Lambda$ pode ser vista no artigo de Overduin e Cooperstock (1998). Nestes modelos a expansão pode ser acelerada como requerido pelas observações de SNe Ia, vejam Chen e Wu (1990), isto 
também resolve o problema da idade em $z=0$ (Calvão et al., 1992). Para um dos modelos mais simples, com $\Lambda(t)=\beta H^{2}$, Birkel e Sarkar (1997) obtiveram $\beta \leq 0$, 13. Já Lima et al. (2000) limitaram $\beta \leq 0,16$, considerando que o parâmetro $\beta$ assume algum valor durante a época dominada pela radiação e pela matéria. Diversos testes cinemáticos tem sido discutidos na literatura para vincular parâmetros cosmológicos a partir de observações de supernovas, diâmetro angular versus redshift, lentes gravitacionais e outros (Calvão et al. (1992); Waga (1993); Waga e Bloomfield Torres (1996); Vishwakarma (2000); Cunha et al. (2002a,b); Alcaniz e Maia (2003); Santos (2007)).

\subsection{Matéria-X}

Outra alternativa à constante cosmológica é uma possível generalização da equação de estado do vácuo, $p=\omega \rho$, onde $\omega<0$ ( $\mathrm{O}$ caso $\omega=-1$ recai na constante cosmológica). No modelo cujo cenário é conhecido por $X(z) C D M$ ambas as componentes do fluído são conservadas separadamente (Turner e White, 1997; Chiba et al., 1997; Alcaniz e Lima, 2001; Kujat et al., 2002; Jain et al., 2003). A equação de estado da componente de energia

escura é $p_{x}=\omega(z) \rho_{x}$. É usual assumir à priori que $\omega(z)=\omega_{o}(1+z)^{n}$. Sendo esta uma forma completamente distinta do que ocorre em modelos de campos escalares, onde $\omega(z)$ é obtido com o campo descrito (Saini et al., 2000; Erickson et al., 2002).

Hoje na literatura existem duas vertentes no estudo da matéria-X, alguns autores estudam o $X C D M$ padrão $(-1 \leq \omega<0$, constante) e outros o XCDM estendido (denominado phantom de energia (Caldwell, R. R., 2002)), no qual o parâmetro $\omega$ pode assumir valores $<-1$. Os dados observacionais mais utilizados para os testes cinemáticos para a distinção entre os modelos são raio-X (Lima et al., 2003), SNe Ia distantes (Garnavich et al., 1998), SNe Ia + RCF (Efstathiou, 1999), SNe Ia + Estruturas de Grande Escala (EGE) (Perlmutter et al., 1999), Estatística de Lentes Gravitacionais (Chae et al., 2002a) e objetos velhos em altos redshift (OVAR) (Lima e Alcaniz, 2000; Alcaniz et al., 2003). Podemos destacar Garnavich et al. (1998), em que, através dos dados de SNe Ia do High-z Supernova Search Team, encontraram $\omega<-0.55$ (95\% c.e.), para modelos planos e $\omega<-0.6$ (95\% c.e.), para geometrias arbitrarias. Estes resultados estão de acordo com o modelo de "concordância cósmica" (Wang et al., 2000).

Lima e Alcaniz (2002) investigaram o diagrama de diâmetro angular $(\theta(z))$ versus 


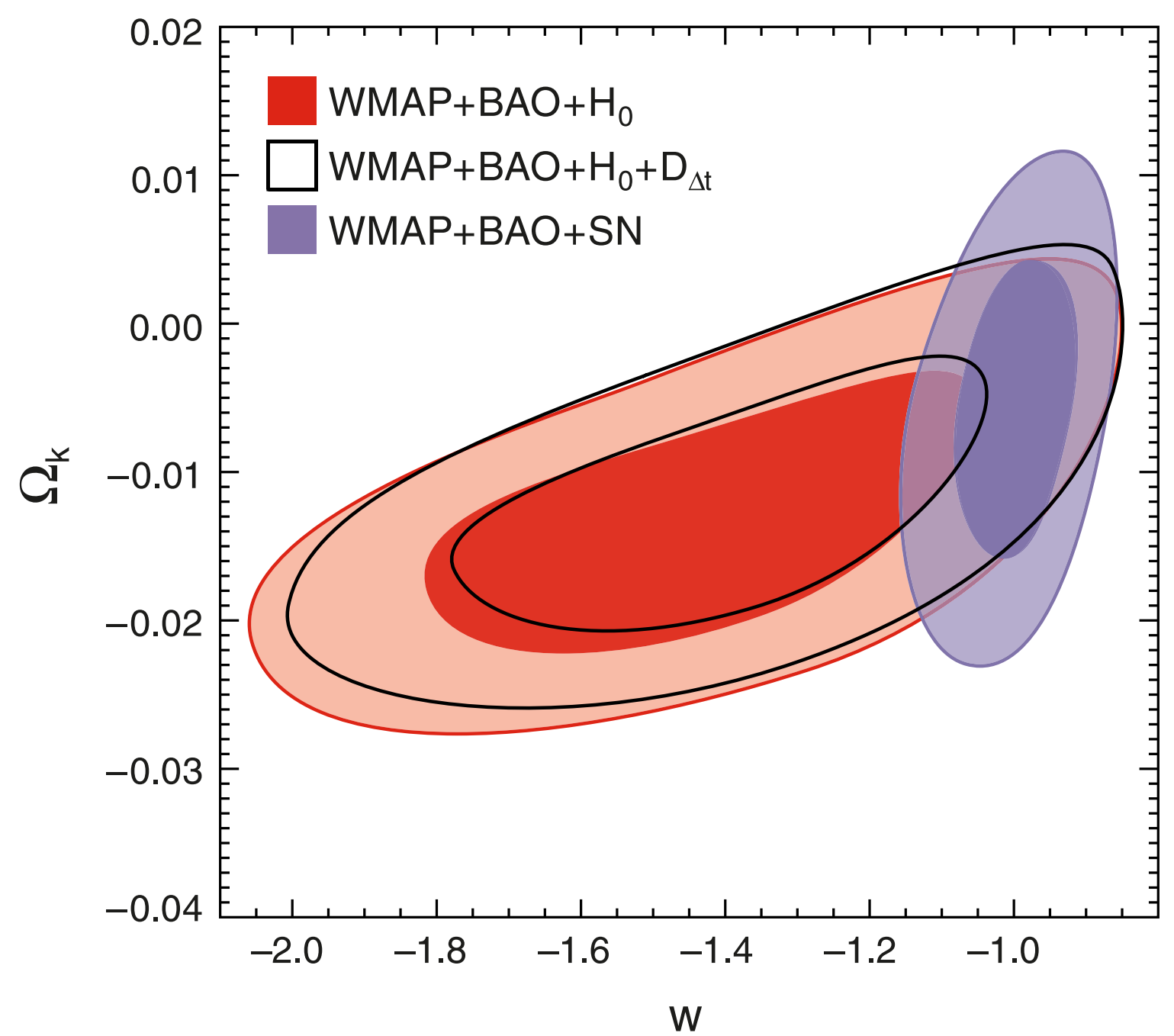

Figura 2.3: Plano $\left(\Omega_{k} \times \omega\right)$. Vínculos no parâmetro da equação de estado $(\omega)$ para um universo combinando os dados do WMAP com outras observações. Vemos que a energia fantasma Phantom $(\omega<-1)$ não pode ser excluída por essas observações (Komatsu et al., 2010).

redshift, utilizando os dados de Gurvits et al. (1999), e delimitaram $-1 \leq \omega \leq-0,5$. Corasaniti e Copeland (2002) obtiveram $-1 \leq \omega \leq-0,93$, usando os dados de SNe Ia e medidas de picos acústicos no espectro RCF. Chae et al. (2002b) utilizou uma análise estatística de Lentes gravitacionais do Cosmic Lens All Sky Survey (CLASS) encontrando $\omega<-0,55_{-0,11}^{+0,18}(68 \%$ c.e.). Jain et al. (2003) utilizaram a separação de imagens na função de distribuição $(\Delta \theta)$ de quasares lentes obtendo $-0,75 \leq \omega \leq-0,42$, para o limite observado $\Omega_{M} \sim 0,2-0$, 4. Já Bean e Melchiorri (2002) obtiveram $\omega<-0,85$ para os dados conjuntos da $\mathrm{RCF}+\mathrm{SNe}$ Ia $+\mathrm{EGE}$, os quais não produzem evidências significativas para $X C D M$, o que conduz a um valor diferente para constante cosmológica. 
Uma conclusão equivalente foi obtida por Schuecker et al. (2003) em análises envolvendo o REFLEX X-ray Cluster e dados de Supernovas, na qual a condição $\omega \geq-1$ foi relaxada.

\subsection{O Caso de Energia Fantasma $(\omega<-1)$}

Modelos do tipo XCDM estendido, ou seja, quando o parâmetro da equação de estado assume valores $\omega<-1$, é usualmente denominado de Energia Fantasma (Phantom Energy). Embora muito discutido na literatura recente, não existe até o presente uma descrição teórica satisfatória da energia fantasma (Lima et al., 2003; Caldwell et al., 2003; Caldwell e Steinhardt, 1998). O modelo mais simples de phantom é motivado por um campo escalar tendo um sinal negativo no termo cinético (Chiba et al., 2000). Algumas versões são também motivadas pela cosmologia de branas (Sahni e Shtanov, 2003; McInnes, 2002; Carroll et al., 2003). Nesses modelos a condição de energia dominante, $|p| \leq \rho$, é sempre violada.

Nos modelos dirigidos pela energia fantasma, a densidade de energia cresce com o tempo, $\rho_{E F} \sim a^{-3(1+\omega)}$, já que $\omega<-1$. Portanto, quando $a \rightarrow \infty$, a densidade de energia diverge. Assim, numa escala de tempo finita, o universo evolui para uma singularidade no futuro denominada de "Big-Rip", uma terminologia introduzida por Caldwell e colaboradores (Caldwell et al., 2003; Caldwell e Steinhardt, 1998). Para um universo plano, o tempo para atingir o Big-Rip é dado por:

$$
\Delta t=t_{\text {rip }}-t_{o}=\frac{2 H_{0}^{-1}}{3|1+\omega|\left(1-\Omega_{M}\right)^{1 / 2}} .
$$

Se $H_{0}=70 \mathrm{Kms}^{-1} M p c^{-1}, \omega=-1,5$ e $\Omega_{M}=0,3$, o tempo para atingir o Big-Rip é dado por $t_{\text {rip }}-t_{0} \sim 22$ bilhões de anos.

Naturalmente, com o crescimento da densidade de energia todos os invariantes de curvatura divergem no futuro e como um resultado, uma segunda era de gravitação quântica deve ocorrer bem antes do "Big-Rip". Isso significa que os mesmos métodos e técnicas utilizadas para estudar a singularidade inicial podem ser adaptadas para o "Big-Rip".

Lima e Alcaniz (2004) estudaram alguns aspectos termodinâmicos da energia fantasma e mostraram que, em geral, seu comportamento termodinâmico é completamente distinto da matéria comum. Em particular, a temperatura cresce ao longo da expansão, $T a^{1+\omega}=$ 
constante, enquanto a entropia é negativa, $S \propto(1+\omega) T^{3} a^{3}$. Isso deveria eliminar o caso da energia fantasma. Porém, González-Díaz e Sigüenza (2004) argumentaram que estados com entropia positiva são obtidos se a temperatura for negativa, de forma que o status teórico da energia fantasma ainda permanece controverso.

Do ponto de vista mais observacional a situação é menos controversa e várias análises independentes baseadas em diferentes observações mostram que a energia fantasma é um bom candidato para explicar o presente estado acelerado do universo. Cunha, Alcaniz e Lima (2004), combinando dados de raios-X de aglomerados e supernovas obtiveram $\omega=-1,29_{-0,792}^{+0,686}$. Hannestad e Mortsell (2002) combinaram RCF + (EGE) + dados de SNe Ia, com $95,4 \%$ c.e., encontrando $-2,68<\omega<-0,78$. Mais recentemente, os resultados do WMAP (Komatsu et al., 2010) se mostraram bastante consistentes com a condição de energia fantasma (ver figura 2.3).

\subsection{Campo Escalar - Quintessência}

A idéia de campo escalar primordial é motivado por modelos da grande unificação da física de partículas de altas energias. Este campo pode ser simplesmente descrito por uma ação da seguinte forma $(\hbar=1)$ :

$$
S=\int d^{4} x \sqrt{-g}\left[\frac{1}{2} g^{\mu \nu} \partial_{\mu} \Phi \partial_{\nu} \Phi-V(\Phi)\right] \quad,
$$

sendo o potencial $V$ uma função do campo $\Phi$ e $g$ o determinante do tensor métrico.

Em um universo em expansão, um campo escalar espacialmente homogêneo com potencial $V(\Phi)$ tem um tensor de energia-momentum diagonal, $T_{\nu}^{\mu}=\left(\rho_{\Phi}, p_{\Phi}, p_{\Phi}, p_{\Phi}\right)$, com densidade de energia e pressão são dadas por (Kolb e Turner, 1990)

$$
\begin{aligned}
& \rho_{\Phi}=\frac{1}{2} \dot{\Phi}^{2}+V(\Phi), \\
& p_{\Phi}=\frac{1}{2} \dot{\Phi}^{2}-V(\Phi) .
\end{aligned}
$$

Implicando num parâmetro da equação de estado do tipo

$$
\omega_{\Phi}=\frac{\frac{1}{2} \dot{\Phi}^{2}-V(\Phi)}{\frac{1}{2} \dot{\Phi}^{2}+V(\Phi)}
$$


que em geral varia com o tempo (ou com o redshift z). Em particular, quando o campo varia lentamente e $\dot{\Phi}^{2} \ll V(\Phi)$, temos $\omega_{\Phi} \sim-1$, e o campo $\Phi$ age equivalentemente a uma constante cosmológica. No entanto, fisicamente estas duas formas de energia são distintas, sendo o campo uma componente dinâmica e a densidade de energia do vácuo constante. É interessante considerar uma componente escura dinâmica como uma alternativa para $\Lambda$, pois um campo escalar deste tipo escapa do seríssimo problema de ajuste fino que inevitavelmente acompanha a constante cosmológica (problema da coincidência cósmica).

A equação de movimento para o campo escalar nos modelos do tipo FRW pode ser obtida das equações de Einstein ou mais diretamente da conservação de energia $\left(T_{; \nu}^{\mu \nu}=0\right)$. Quando o campo é minimamente acoplado com a gravidade, obedece a seguinte equação (Kolb e Turner, 1990)

$$
\ddot{\Phi}+3 H \dot{\Phi}+V^{\prime}(\Phi)=0
$$

onde o ponto $\left({ }^{*}\right)$ significa derivadas em relação ao tempo e a linha $\left({ }^{\prime}\right)$ indica derivada com respeito ao campo $\phi$. A expressão acima é análoga à equação de um oscilador harmônico com a taxa de expansão $H$ desempenhando o papel de um coeficiente de atrito. Para potenciais genéricos, o campo $\Phi$ será superamortecido (aproximadamente constante) para $H>\sqrt{V^{\prime \prime}(\Phi)}$ e subamortecido (livre para rolar até o mínimo do potencial) quando $H<$ $\sqrt{V^{\prime \prime}(\Phi)}$.

Modelos de campos escalares foram inicialmente utilizados para descrever a expansão quase exponencial no início do universo (período inflacionário), quando a densidade de energia do universo é dominada pelo potencial $V(\Phi)$. Durante o regime da inflação o universo esfria adiabaticamente chegando a uma temperatura extremamente baixa. Contudo, para que a nucleossíntese cosmológica se realize é necessário reaquecê-lo, o que é feito as expensas da energia do campo durante o seu decaimento em partículas relativísticas. No reaquecimento, que ocorre devido ao seu acoplamento com os outros campos de matéria, o campo $\Phi$ oscila rapidamente e decai produzindo toda a entropia do nosso universo. O processo efetivamente termina quando sua densidade de energia $\rho_{\Phi}$ assume valores extremamente pequenos ou zero.

Podemos também imaginar que a evolução recente de $\rho_{\Phi}$ é lenta. Se for mais lenta que a evolução na densidade de massa da matéria, chegará um tempo em que $\rho_{\Phi}$ dominará 
novamente, e o universo se comportará como tendo uma constante cosmológica efetiva. Esta também parece ser a maneira mais simples e natural de descrever a Quintessência (Caldwell, 2000; Peebles e Ratra, 2003). Uma grande questão ainda em aberto é saber se a Quintessência que dirige o presente estágio acelerado é o mesmo campo que gerou a inflação.

Um exemplo interessante de cosmologias com campo escalar primordial foi proposto por Peebles e Ratra (2003). Nesse cenário, a parte do campo $\Phi$ chamado inflaton, é responsável pela inflação e convertida em entropia no final do período inflacionário. A parte restante decresce muito mais lentamente até o mínimo do potencial e vai acelerar o universo no final da era da matéria. O potencial assume a seguinte forma

$$
V(\Phi)=\kappa / \Phi^{\alpha}
$$

onde a constante $\kappa$ tem dimensões de massa elevada a potência de $\alpha+4$. O parâmetro da equação de estado para a época dominada por matéria é independente do tempo.

$$
\omega_{\Phi}=-\frac{2}{\alpha+2}
$$

e modela uma constante cosmológica pura para $\alpha \rightarrow 0$. No período em que a densidade dessa energia escura começa a ter uma contribuição apreciável para a taxa de expansão o parâmetro $\omega_{\Phi}$ começa a evoluir, e o uso de $\omega_{\Phi}$ como uma constante no modelo pode tornase um erro. As previsões dos modelos de Quintessência á luz dos mais diferentes testes observacionais vem sendo estudados ao longo dos últimos anos (Caldwell, 2000; Peebles e Ratra, 2003; Carvalho et al., 2006)

\subsection{Gás Tipo Chaplygin}

Neste cenário alternativo, a descrição da matéria escura fria e energia escura é unificada, reduzindo o setor escuro a apenas uma componente. Em Wetterich (2002) essa matéria escura foi proposta como um tipo de quintessência. Já Padmanabhan e Choudhury (2003) investigaram algumas possibilidades via teoria de cordas modificada pelo campo tachiônico. Contudo, parece ser mais promissora a tentativa sugerida por Kamenshchik et al. (2001) e desenvolvida por Bilic et al. (2002) e Bento et al. (2002). Esta refere-se a um fluido 
exótico, o então chamado gás de Chaplygin (gC), cuja equação de estado é

$$
p_{g C}=-A / \rho^{\alpha},
$$

onde $A$ e $\alpha=1$ são constantes positivas. No caso de $\alpha \neq 1$ temos o gás de Chaplygin generalizado (Bento et al., 2002). No caso $\alpha=0$, temos o cenário $\Lambda$ CDM usual.

O fenômeno mais interessante nesse cenário é que o gás de Chaplygin pode interpolar naturalmente entre a matéria não relativística e um regime de energia escura (Bilic et al., 2002; Bento et al., 2002). Fabris et al. (2002) investigaram algumas consequências deste cenário utilizando os dados de Supernovas tipo Ia. Estes resultados indicam que a cosmologia dominada completamente por gás Chaplygin é favorecida em comparação com os modelos $\Lambda$ CDM. Já Avelino et al. (2003) usaram um número maior de SNe Ia e a forma do espectro de potência da matéria, para demonstrar que estes dados restringem o modelo para $\Lambda$ CDM, enquanto Bento et al. (Bento et al., 2003a,b) mostraram que a localização dos picos da RCF fornecem vínculos nos parâmetros livres do modelo. Enquanto Dev, Alcaniz e Jain (2003) e Alcaniz, Jain e Dev (2003) investigaram o vínculo na equação de estado do gás Chaplygin utilizando estatística de lentes gravitacionais fortes em estimativas de idade em altos- $z$. Vínculos envolvendo outras observações astrofísicas tem sido discutidos por diversos autores (Bento et al., 2003b; Silva e Bertolami, 2003; Makler et al., 2003; Carturan e Finelli, 2003; Amendola et al., 2003; Cunha et al., 2004).

\subsection{Gás de Chaplygin Simplificado}

Além do parâmetro de Hubble $H_{0}$ temos mais 3 parâmetros $\left(\alpha, A_{s}, \Omega_{M}\right)$ para o gás de Chaplygin, impondo a condição de universo plano. Neste caso, é interessante reduzir os parãmetros do gás de Chaplygin, se basando em alguma condição física razoável.

Para tal, primeiramente notemos que a constante $A_{s}$ aparecendo nas expressões acima conduz a uma informação básica que vem do parâmetro original $A$, desde que apenas $A_{s}$ e $\alpha$ aparecem explicitamente nas equações de FRW do movimento. Por outro lado, a velocidade do som adiabática de Chaplygin é

$$
v_{s}^{2}=\frac{d p}{d \rho}=\alpha A / \rho_{C}^{1+\alpha}
$$


que deve ser positivo definido para um gás bem comportado (zero no limite do caso de poeira). Note também que para o tempo presente a velocidade do som adiabática Chaplygin é $v_{s 0}^{2}=\alpha A / \rho_{C_{0}}^{1+\alpha}$, e combinando com a expressão relacionando as constantes $A$ e $A_{s}$ encontramos

$$
v_{s}^{2}=\alpha A / \rho_{C 0}^{1+\alpha}=\alpha A_{s}
$$

Portanto, se $A_{s}$ é uma função de $\alpha$ podemos além de reduzir o número de parâmetros livres, a positividade de $v_{s}^{2}$ passa a ser naturalmente garantida. Vemos então que a mais simples escolha é $A_{s}=\alpha$. Neste caso, $v_{s o}^{2}=\alpha^{2}$, ou mais geralmente, $v_{s}^{2}=\alpha^{2} \rho_{C 0}\left(\rho_{C 0} / \rho\right)^{\alpha}$, onde a equação de estado do gás de Chaplygin generalizado torna-se

$$
p_{C}=-\alpha \rho_{C 0}\left(\frac{\rho_{C 0}}{\rho_{C}}\right)^{\alpha}
$$

tal que, no presente, o gás simplificado "imita" a matéria-X $\left(p_{C 0}=-\alpha \rho_{C 0}\right)$, sendo completamente caracterizada pelo parâmetro $\alpha$. Um universo atualmente acelerado pode ser obtido e seu comportamento é tipo um fluído sem pressão para grandes valores de $z(q(z) \geq 0)$, assim como uma matéria-X para baixos redshifts tendendo a acelerar a expansão. Se $\alpha=1$, o comportamento dinâmico atual é o mesmo de uma constante cosmológica. Note também que a equação básica de FRW para o modelo simplificado deve ser escrita como

$$
\left(\frac{\dot{a}}{a}\right)^{2}=H_{0}^{2}\left\{\Omega_{M}\left(\frac{a_{0}}{a}\right)^{3}+\left(1-\Omega_{M}\right)\left[\alpha+(1-\alpha)\left(\frac{a_{0}}{a}\right)^{3(\alpha+1)}\right]^{\frac{1}{\alpha+1}}\right\},
$$

mostrando que o parâmetro $\alpha$ é a única constante desconhecida relacionada com o modelo de gás de Chaplygin simplificado.

De acordo com o princípio da "navalha de Okkam", postulamos que $A_{s}$ é uma função de $\alpha$ e por simplicidade tomamos $A_{s}=\alpha$. Desta forma, obtemos um modelo cosmológico mais facilmente falseável pelas observações cosmológicas atuais.

Em síntese, ao admitirmos a existência de uma componente exótica responsável pela aceleração cósmica - cuja equação de estado pode ou não depender do tempo - nos deparamos com mais duas questões de princípio que precisam ser devidamente consideradas. A primeira delas é conhecida como o problema da coincidência cósmica e consiste em entender porque o universo começou a se expandir de forma acelerada só mais recentemente. Em outras palavras, por que as densidades de energia da matéria escura e da energia escura são 
da mesma ordem de magnitude? Por exemplo, se o valor de $\Lambda$ fosse dez vezes maior que a sua estimativa atual, o universo já teria iniciado a aceleração há muito mais tempo atrás. Neste caso não haveria tempo para formar estruturas como galáxias e seus aglomerados. Se $\Lambda$ fosse dez vezes menor, nós não observaríamos a aceleração cósmica e esta só poderia ser detectada em um futuro bem distante.

As evidências de que o universo possui uma componente com pressão negativa, uniformemente distribuída e que contribui com $\approx 70 \%$ para a densidade total de energia se mostram extremamente convincentes. Contudo, a quantidade de candidatos teóricos apresentados acima significa que pouco sabemos sobre a natureza dessa componente. Na verdade, alguns cosmólogos questionam sua própria existência e exploram a possibilidade de que a aceleração cósmica seja fruto de uma nova teoria de gravitação. Há entre os cosmólogos um consenso de que futuros avanços dependem de novas observações bem como de uma compreensão mais profunda de física fundamental.

$\mathrm{Na}$ fase atual da cosmologia é importante continuar buscando alternativas teóricas capazes de explicar a aceleração cósmica, por mais estranhas ou exóticas que elas possam parecer. Existe uma crença geral na comunidade de que ainda nessa década, grandes avanços serão alcançados no entendimento da natureza da energia escura ou, de uma forma mais ampla, na substância ou mecanismo que gera a aceleração cósmica.

Nesse contexto, uma questão importante é a seguinte: é possível acelerar o universo sem energia escura?

Em particular, seria interessante saber se o chamado setor escuro pode ser reduzido a uma componente, nesse caso, constituída apenas pela matéria escura, principalmente pelo fato de que atualmente o status da matéria escura é maior do que o da energia escura (ver seção 1.4.1 ). Existem pelo menos duas maneiras de acelerar o universo considerando apenas a presença da matéria escura. A primeira delas nos leva para as chamadas teorias $f(R)$, enquanto a segunda (mais relacionada com esta dissertação), é consequência do processo de criação gravitacional da matéria escura.

(i) Teorias $f(R)$. Em tais abordagens são verificadas as possibilidades de modificações da TRG através da substituição do escalar de curvatura $R$ por uma função geral $f(R)$ na ação de Einstein-Hilbert (equação (1.14)). Assim, a ação de Einstein-Hilbert modificada tem a forma: 


$$
I_{E H M}=\frac{1}{16 \pi G} \int d^{4} x \sqrt{-g} f(R) .
$$

Embora esta abordagem descarta a necessidade de energia escura para a aceleração do universo, a grande liberdade na forma da função $f(R)$ dificulta o estabelecimento de limites teóricos e observacionais (Santos et al., 2007; Pereira et al., 2009)

(ii) Teorias com criação de matéria escura. Em tais teorias, o processo de criação de matéria escura é descrito por uma pressão negativa, requisito essencial para um estágio acelerado (Lima et al., 1991; Calvão et al., 1992; Lima e Germano, 1992). Estas teorias, constituem o tema central dessa dissertação e serão discutidas em detalhe nos próximos capítulos. 


\section{Capítulo 3}

\section{Modelos Acelerados com Criação de Matéria}

A questão sobre a origem dos constituintes do universo vem sendo vastamente investigada na literatura através de alguns mecanismos possíveis de criação de matéria no contexto cosmológico.

Nos anos 1970, Tryon (1973) e, independentemente, Fomin (1975) propuseram a idéia de que o universo pudesse ter surgido de uma flutuação do vácuo. Neste caso, as leis da física exigem que todas as quantidades conservadas tenham valor total zero, em especial a energia, na qual a existência do conteúdo material seria compensada pela própria energia negativa da gravidade. O surgimento do universo se daria então por uma flutuação quântica do vácuo, cuja duração é restrita pela relação de incerteza de Heisenberg $\Delta t \cdot \Delta E \sim \hbar$. Ainda que em tal formulação não fosse apresentada nenhum cenário específico, admitiu-se nestes trabalhos que o universo poderia ser totalmente compreendido dentro dos paradigmas da ciência convencional, incluindo a sua origem.

A questão fora abordada microscopicamente nos trabalhos de Parker (1968) e Fulling et al. (1974) que mostraram que os constituintes elementares do universo podem ser produzidos quanticamente por um campo gravitacional variável de um universo em expansão.

Destacamos ainda a abordagem de Zeldovich (1981), que sugeriu um mecanismo efetivo com o intuito de descrever um modelo de universo com criação de matéria que evita o problema da singularidade inicial.

No contexto da inflação cósmica, existem muitas investigações sobre o papel que processos irreversíveis poderiam ter neste cenário (Turner, 1983; Barrow, 1986, 1988; Lima et al., 1988). A presença da viscosidade volumar (criação de matéria), tem como resultado dinâmico um termo de pressão negativa, podendo ser portanto o processo que resulta na 
inflação.

A questão fundamental a ser respondida até então seria como o mecanismo de criação de matéria poderia ser incorporado consistentemente às equações de campo de Einstein. Prigogine, Geheniau, Gunzig e Nardone (1989) reinterpretaram o tensor de momento-energia , modificando a relação usual nas equações de campo de Einstein através da criação irreversível de matéria às custas do campo gravitacional, através de um formalismos baseado na termodinâmica de sistemas abertos. Neste caso, a origem do universo ocorreria por uma instabilidade termodinâmica ao invés de uma singularidade inicial. Este mecanismo foi posteriormente generalizado por Lima, Calvão e Waga (1991) e Lima e Germano (1992) em uma formulação manifestamente covariante. Nestes trabalhos, mostrou-se que, embora viscosidade volumar e pressão de criação sejam processos dissipativos que resultam em um termo de pressão negativa a nível das equações de Einstein, tratam-se de mecanismos distintos do ponto de vista termodinâmico.

Neste capítulo, discutiremos a dinâmica de um universo em que o número de partículas de matéria escura fria (cold dark matter - CDM) não permanece constante. A abordagem se deu através da aplicação da termodinâmica de não-equilíbrio para a cosmologia, permitindo que haja simultaneamente a criação de partículas de CDM e uma fonte de entropia (Prigogine et al., 1989; Lima et al., 1991; Calvão et al., 1992).

No contexto das recentes observações de supernovas tipo Ia (SNe Ia) (Riess et al., 1998; Perlmutter, 1999), destacamos esta classe de modelos como uma explicação alternativa para o atual estágio acelerado em que o universo passa, em que a presença de um termo de constante cosmológica não nulo, assim como de qualquer outro tipo de fluido exótico, é desnecessário para a concordância com as observações. Neste caso, apenas o processo de criação de matéria é suficiente para a existência de um termo de pressão negativa (gravitacionalmente repulsiva), denominada de pressão de criação, evitando o problema da coincidência cósmica.

\subsection{Criação de Matéria Escura no Universo: Formulação Termodinâmica}

Os estados termodinâmicos de um fluido relativístico simples podem ser descrito por algumas variáveis macroscópicas básicas: o tensor momento-energia $T^{\alpha \beta}$, o vetor fluxo de partículas $N^{\alpha}$ e o vetor fluxo de entropia $S^{\alpha}$ (Calvão et al., 1992; Lima et al., 1991). 
Nesta formulação, o tensor momento-energia para um fluido isotrópico tem a forma

$$
T^{\mu \nu}=(\rho+P) u^{\mu} u^{\nu}-P g^{\mu \nu}
$$

onde $\rho=\rho_{d m}+\rho_{b}$ é a densidade total de matéria ( matéria escura + matéria bariônica) e a pressão dinâmica $\mathrm{P}$ na equação (3.1) é decomposta em

$$
P=p+p_{c}
$$

onde p é a pressão de equilíbrio termostático e $p_{c}$ corresponde a um termo de correção devido a processos dissipativos no fluido (viscosidade volumar ou criação de matéria).

Temos ainda que o tensor em (3.1) satisfaz à lei de conservação:

$$
T^{\alpha \beta} ;_{\beta}=0
$$

Seja $N$ o número total de partículas contidas em um dado volume comóvel $V$. A variação de $N$ é descrita por:

$$
\dot{N} / N \equiv(n \dot{V}) / n V=\dot{n} / n+\Theta \equiv \Gamma
$$

sendo $n$ a densidade de partículas, $\Theta \equiv \dot{V} / V$ a expansão do fluido e $\Gamma$ a taxa, em unidades de $s^{-1}$, com que partículas são criadas $(\Gamma>0)$ ou destruidas $(\Gamma<0)$. É apropriado então definirmos o vetor fluxo de partículas:

$$
N^{\alpha}=n u^{\alpha}
$$

que satisfaz à equação de balanço:

$$
N^{\alpha} ;_{\alpha}=n \Gamma
$$

de modo que a equação (3.6) é a forma covariante da equação (3.4). Na métrica de FRW, a equação (3.6) é dada explicitamente por (Lima et al., 1996):

$$
\dot{n}+3 H n=n \Gamma \text {. }
$$

Analogamente, definimos o vetor fluxo de entropia, dado por 


$$
S^{\alpha}=n \sigma u^{\alpha}
$$

onde $\sigma$ é a entropia específica (por partícula). Uma vez que, pela segunda lei da termodinâmica, a variação da entropia total de um sistema fechado satisfaz $d S=d(N \sigma) \geq 0$, temos que (Calvão et al., 1992; Lima et al., 1991)

$$
S^{\alpha} ;_{\alpha}=(\dot{n} \sigma)+3 H n \sigma \geq 0 .
$$

Desta forma, caracterizamos o processo de criação de partículas como um processo irreversível. Além disso, a formulação mostrada neste capítulo se diferencia essencialmente de outras cosmologias dissipativas pois, embora haja semelhanças com a descrição de um cenário com viscosidade volumar (c.f. equações (3.1), (3.2)), o aumento de entropia neste caso ocorre apenas pela criação de matéria.

\subsection{Dinâmica da Criação de Matéria Escura}

No contexto da criação de matéria escura, o conjunto de equações (1.20) e (1.21) toma a forma

$$
\begin{gathered}
\chi \rho=3 \frac{\dot{a}^{2}}{a^{2}}+3 \frac{k}{a^{2}} \\
\chi\left(p+p_{c}\right)=-2 \frac{\ddot{a}}{a}-\frac{\dot{a}^{2}}{a^{2}}-\frac{k}{a^{2}},
\end{gathered}
$$

onde, novamente, $p_{c}$ representa a pressão de criação.

Da conservação do tensor (3.1) obtemos :

$$
\dot{\rho}+\left(\rho+p+p_{c}\right) \Theta=0 .
$$

Para este sistema, a lei de Gibbs toma a forma

$$
n T d \sigma=d \rho-\frac{\rho+p}{n} d n
$$

onde $T$ é a temperatura.

Das equações acima, temos que 


$$
S^{\alpha} ;{ }_{\alpha}=n \sigma \Gamma+n \dot{\sigma}=-\frac{3 H p_{c}}{T}-\frac{\mu n \Gamma}{T} \geq 0,
$$

onde utilizamos que, na métrica de FRW (1.17), $\Theta=3 H$ e que o potencial químico é dado pela relação de Euler:

$$
\mu=\frac{\rho+p}{n}-T \sigma
$$

Entende-se que no caso "adiabático" de criação matéria gravitacional a entropia específica se mantém constante $(\dot{\sigma}=0)$ enquanto seu valor global pode ser aumentado. Uma consequência importante disto é que, por (3.14),

$$
S^{\alpha}{ }_{\alpha}=n \sigma \Gamma \geq 0
$$

ou seja, como consequência da segunda lei da termodinâmica, a taxa de criação de partículas de CDM só pode ser positiva ou nula (neste último caso, correspondendo a um fluido perfeito).

É importante ainda determinarmos a relação entre a pressão de criação e a taxa de criação de partículas. Reescrevendo (3.15), ficamos com:

$$
n \sigma=\frac{\rho+p}{T}-\frac{\mu n}{T}
$$

e aplicando a condição de "adiabaticidade" $(\dot{\sigma}=0)$ em (3.14):

$$
\frac{3 H p_{c}}{T}=-n \sigma \Gamma-\frac{\mu n \Gamma}{T} .
$$

Assim, pela combinação de (3.17) e (3.18), a pressão de criação se expressa como (Prigogine et al., 1989; Lima et al., 1991; Calvão et al., 1992):

$$
p_{c}=-\frac{\rho+p}{3 H} \Gamma
$$

Da equação acima, devemos observar que, uma vez que a positividade de $\Gamma$ é garantida por (3.16), se $\rho+p>0$ então, necessariamente, $p_{c} \leq 0$. Portanto, para um fluido normal $(\rho+p>0)$, a pressão de criação sempre contribui para produzir aceleração. 
Naturalmente, neste tipo de cenário o comportamento do fator de escala também é afetado pela taxa de criação. Para exemplificar, consideremos um modelo de universo composto por criação de partículas e um fluido com equação de estado

$$
p=\omega \rho,
$$

verificamos que as equações (3.10), (3.11) e (3.19), resultam em (Lima et al., 2008):

$$
2 a \ddot{a}+\left[(1+3 \omega)-(1+\omega) \frac{\Gamma}{H}\right]\left(\dot{a}^{2}+k\right)=0,
$$

onde, no caso particular $\Gamma=0$, recuperamos a equação que rege a evolução do fator de escala para um fluido perfeito de uma componente.

\subsection{Lei de Evolução da Temperatura}

Neste ponto, deduziremos a lei de evolução da temperatura do universo quando processos de criação são levados em conta (Calvão et al., 1992; Silva et al., 2002).

Primeiramente, adotaremos como variáveis termodinâmicas básicas a densidade de partículas $n$ e a temperatura $T$, ou seja,

$$
\begin{aligned}
& \rho=\rho(T, n), \\
& p=p(T, n),
\end{aligned}
$$

de forma que,

$$
\dot{\rho}=\left(\frac{\partial \rho}{\partial T}\right)_{n} \dot{T}+\left(\frac{\partial \rho}{\partial n}\right)_{T} \dot{n}
$$

Da lei de conservação de energia (3.12) e do balanço de partículas (3.6), obtemos então que

$$
\dot{T}=-\frac{1}{(\partial \rho / \partial T)_{n}}\left\{\left[\rho+p-n\left(\frac{\partial \rho}{\partial n}\right)_{T}\right]+p_{c} \Theta+\left(\frac{\partial \rho}{\partial n}\right)_{T} n \Gamma\right\} .
$$

Utilizando a equação (3.13) e o fato de que $\sigma$ é uma diferencial exata: 


$$
T\left(\frac{\partial p}{\partial T}\right)_{n}=\rho+p-n\left(\frac{\partial \rho}{\partial n}\right)_{T} .
$$

Combinando as equações (3.25) e (3.26), chegamos a

$$
\frac{\dot{T}}{T}=-\left(\frac{\partial p}{\partial \rho}\right)_{n} \Theta-\frac{p_{c} \Theta+(\partial \rho / \partial n)_{T} n \Gamma}{T(\partial \rho / \partial T)_{n}} .
$$

Tomando o caso particular, onde $\Gamma=p_{c}=0$, obtemos

$$
\frac{\dot{T}}{T}=-\left(\frac{\partial p}{\partial \rho}\right)_{n} \frac{\dot{n}}{n}
$$

que é o resultado para a lei de evolução de temperatura no caso de um fluido simples em equilíbrio, com conservação do número de partículas (Weinberg, 1971; Lima e Tiomno, 1989).

No capítulo a seguir, o último desta dissertação, discutiremos um novo cenário cosmológico sem energia escura, baseado na criação de partículas induzida gravitacionalmente pela expansão universal. 


\section{Capítulo 4}

\section{Um Novo Modelo com Criação de Matéria}

No contexto da Teoria da Relatividade Geral, as fortes evidências da expansão acelerada do universo fornecidas pelas observações de Supernovas do Tipo Ia (SNe Ia) (Riess et al., 1998; Perlmutter, 1999; Kowalski et al., 2008) são usualmente explicadas por meio da existência de uma nova componente escura, um fluido exótico com pressão negativa conhecido como energia escura.

Mostramos no capítulo 2 que, além das diversas possibilidades de fluidos cosmológicos representando a energia escura, o atual estágio acelerado do universo pode ser explicado sem a adição de alguma componente extra. Em particular, como visto no capítulo 3, a aceleração pode ser explicada através de mecanismos relacionados à produção quântica de partículas em um universo em expansão.

Cosmologias com criação de matéria estão contidas neste contexto. Embora ainda não tenha sido criada uma teoria quântica da gravitação que possibilite explicar o fenômeno de criação por primeiros princípios, é possível analisarmos os efeitos que o processo de criação tem sobre a dinâmica cosmológica através da taxa de criação, $\Gamma$.

Neste capítulo, propomos uma nova classe de modelos cosmológicos somente com matéria escura fria e acelerada através do mecanismo de criação de partículas às custas do campo gravitacional. Ressaltamos que o modelo proposto é capaz de ajustar os dados observacionais de Supernovas Tipo Ia tão bem quanto o modelo padrão mas sem a necessidade de uma constante cosmológica não nula. Na verdade, a pressão negativa, atribuída geralmente à existência de uma componente energia escura, aparece como consequência natural do cenário descrito a seguir. 


\subsection{Modelo CCDM (Creation Cold Dark Matter)}

Proporemos aqui uma nova classe de modelos definidos através da escolha da taxa de criação de partículas, $\Gamma$. A princípio, podemos pensar que a escolha mais natural seria uma taxa de criação de partículas que não favorecesse nenhuma época em especial durante a evolução do universo, ou seja,

$$
\Gamma \propto H
$$

onde $H$ é o parâmetro de Hubble.

Na literatura recente, cenários com criação de matéria escura em que $\Gamma=3 \beta H$, sendo $\beta$ função do tempo, vêm sendo investigados (Lima et al., 2008; Steigman et al., 2009). Lima et al. (2008) mostrou que modelos CCDM resolvem o problema da idade e são genericamente capazes de explicar as observações de SNe Ia.

Steigman et al. (2009) incluiram bárions no modelo assim como testes da evolução desta classe de modelos em altos redshifts usando o vínculo sobre $z_{e q}$, o redshift da época da equipartição matéria - radiação, dado pelos vínculos do WMAP. Esta comparação revelou uma tensão entre os vínculos em altos redshifts da RCF sobre $z_{e q}$ e os dados de SN Ia em baixos redshifts, desafiando a viabilidade desta classe de modelos. Um problema adicional está relacionado à dificuldade matemática encontrada quando uma componente bariônica é adicionada na cosmologia proposta por Lima, Silva e Santos (2008). Na verdade, no cenário mais interessante discutido por Steigman, Santos e Lima (2009), as comparações com as observações somente se tornam possíveis após expandir o parâmetro de Hubble em dois regimes, a saber, em altos e baixos redshifts

Por outro lado, a dificuldade mais essencial dos modelos CCDM vem do fato de que todos eles são planos $\left(\Omega_{d m}+\Omega_{b}=1\right)$, porém não sendo claro como contribuem para os dados de aglomerados que, através de um grande conjunto de observações, se mostram consistentes com $\Omega_{d m}+\Omega_{b} \sim 0.3$ (Allen, 2002; Lima et al., 2003; Rapetti et al., 2008; Vikhlinin et al., 2009). Em particular, isto significa que a seguinte questão desafia os cenários CCDM: Como a taxa de criação afeta a presente quantidade de matéria de forma que exista um parâmetro de densidade de matéria efetivo compatível com as estimativas observacionais? 
No que se segue, mostramos que todos estes inconvenientes podem ser resolvidos através de uma escolha razoável da taxa de criação, $\Gamma$.

Primeiramente, observemos que a aceleração é um fenômeno mais recente na história cósmica, ou seja, a aceleração é suprimida durante a fase da radiação. Isto pode ser obtido ao considerarmos que $\beta(t)$ é uma função inversamente proporcional à densidade de energia de matéria escura. Uma vez que esta é uma quantidade adimensional, sua dependência deve envolver alguma razão com a densidade de energia de matéria escura. Mais especificamente, vamos considerar a seguinte taxa de criação:

$$
\Gamma=3 \alpha\left(\frac{\rho_{c 0}}{\rho_{d m}}\right) H,
$$

onde $\alpha$ é uma constante de proporcionalidade, $\rho_{c 0}$ é o valor atual da densidade crítica, e o fator ' 3 ' foi introduzido por conveniência matemática. Com esta escolha de $\Gamma$, a pressão de criação (equação (3.19)) assume a forma:

$$
p_{c}=-\alpha \rho_{c 0}
$$

Da combinação entre a expressão da conservação de energia para a matéria escura (equação (3.12)) com a equação (4.2), obtemos

$$
\dot{\rho}_{d m}+3 H \rho_{d m}=\Gamma \rho_{d m} \equiv 3 \alpha \rho_{c 0} H
$$

que, ao ser integrada, nos dá como solução para $\rho_{d m}$

$$
\rho_{d m}=\left(\rho_{d m 0}-\alpha \rho_{c 0}\right)\left(\frac{a_{0}}{a}\right)^{3}+\alpha \rho_{c 0}
$$

ou, em termos do redshift, $1+z=\left(a_{0} / a\right)$,

$$
\rho_{d m}=\left(\rho_{d m 0}-\alpha \rho_{c 0}\right)(1+z)^{3}+\alpha \rho_{c 0}
$$

Neste cenário, a solução para as leis de conservação de energia para a radiação e bárions se mantêm iguais às usuais

$$
\begin{aligned}
& \rho_{r}=\rho_{r 0}(1+z)^{4}, \\
& \rho_{b}=\rho_{b 0}(1+z)^{3} .
\end{aligned}
$$


Inserindo estas expressões nas equações de Friedmann (equações (3.10) e (3.11)), temos que

$$
\left(\frac{H}{H_{0}}\right)^{2}=\left(\Omega_{m}-\alpha\right)(1+z)^{3}+\alpha+\left(1-\Omega_{m}\right)(1+z)^{2},
$$

onde a componente de radiação foi desprezada e utilizamos a seguinte definição:

$$
\Omega_{m} \equiv \Omega_{d m}+\Omega_{b}
$$

além da condição de normalização

$$
\Omega_{k}=1-\Omega_{m}
$$

A semelhança com o modelo $\Lambda \mathrm{CDM}$ é evidente, ainda que o modelo CCDM só possua uma componente escura. Na verdade, lembrando que o parâmetro de Hubble para o modelo $\Lambda \mathrm{CDM}$ tem a forma

$$
\left(\frac{H_{\Lambda C D M}}{H_{0}}\right)^{2}=\Omega_{m}(1+z)^{3}+\Omega_{\Lambda}+\left(1-\Omega_{m}-\Omega_{\Lambda}\right)(1+z)^{2},
$$

vemos que os modelos têm o mesmo parâmetro de Hubble $H(z)$, com $\alpha$ tendo o papel dinâmico de $\Omega_{\Lambda}$ e $\Omega_{m}$ sendo substituído por $\Omega_{m}-\alpha$. Esta relação se torna ainda mais clara quando definimos um parâmetro de densidade efetivo da matéria, como

$$
\Omega_{m e f f} \equiv \Omega_{m}-\alpha
$$

e o inserimos na expressão (4.8) para o modelo CCDM.

De forma ainda mais direta, esta equivalência pode ser observada através da equação de evolução do fator de escala cósmico. Introduzindo a expressão da pressão de criação $p_{c}$ (equação (3.19)) na segunda das equações de Friedmann, obtemos:

$$
2 a \ddot{a}+\dot{a}^{2}+k-3 \alpha H_{0}^{2} a^{2}=0,
$$

que pode ser comparada com a expressão

$$
2 a \ddot{a}+\dot{a}^{2}+k-\Lambda a^{2}=0,
$$


proveniente do modelo $\Lambda$ CDM.

As equações (4.13) e (4.14) mostram que os dois modelos terão o mesmo comportamento dinâmico ao identificarmos o parâmetro de criação através da expressão $\alpha=\Lambda / 3 H_{0}{ }^{2} \equiv$ $\Omega_{\Lambda}$, exatamente o mesmo resultado deverivado anteriormente com base no parâmetro de Hubble, $H(z)$.

Porém, embora os modelos CCDM e $\Lambda$ CDM possuam a mesma história dinâmica, estas cosmologias se baseiam em diferentes hipóteses iniciais e, portanto podem ser diferenciadas pelas observações atuais. Do ponto de vista matemático, isto ocorre devido ao papel especial desempenhado pelo parâmetro $\alpha$ nas equações do modelo CCDM. Em particular, a positividade do parâmetro de densidade da matéria e do parâmetro de Hubble implicam que, em altos redshifts o parâmetro de criação deve satisfazer a

$$
\alpha \leq \Omega_{m}
$$

condição que não possui um análogo dentro do modelo $\Lambda$ CDM.

Além disso, é possível que a dependência do redshift devido à contribuição envolvendo o parâmetro $\alpha$, a saber, $\alpha\left(1-(1+z)^{3}\right)$ modifique levemente as previsões envolvendo a evolução de pequenas pertubações e o problema de formação de estruturas.

Quando impomos que a seção espacial do universo seja plana, como previsto pela inflação e sugerido pelos dados de RCF, temos que $\Omega_{m}=1$ e a equação (4.8) se reduz a

$$
\left(\frac{H}{H_{0}}\right)^{2}=(1-\alpha)(1+z)^{3}+\alpha,
$$

sendo $\alpha$ o único parâmetro livre, além de $H_{0}$, exatamente como no modelo padrão, $\Lambda$ CDM plano. Notemos que, neste caso, $\Omega_{m e f f}=1-\alpha$.

\subsection{Vínculos de Supernovas e Redshift de Transição}

Discutiremos agora os vínculos obtidos através dos dados de SNe Ia sobre a classe de modelo CCDM proposta neste trabalho.

Nas discussões a seguir, consideramos tanto o cenário curvo como o plano. A princípio, como $H_{0}$ pode ser determinado via lei de Hubble, o modelo possui apenas dois parâmetros independentes, a saber $\alpha$ e $\Omega_{m}$ ou, equivalentemente, $\Omega_{m e f f}$, conforme a equação (4.8). 
O módulo de distância para uma supernova no redshift z, dado o conjunto de parâmetros s, é

$$
\mu_{p}(z \mid \mathbf{s})=m-M=5 \log d_{l}+25,
$$

onde $m$ e $M$ são, respectivamente, as magnitudes aparentes e absolutas, o conjunto de parâmetros é $\mathbf{s} \equiv\left(H_{0}, \alpha, \Omega_{m}\right)$, e $d_{l}$ é a distância de luminosidade (em unidades de Mpc),

$$
d_{l}=c(1+z) \int_{0}^{z} \frac{d z^{\prime}}{H\left(z^{\prime} ; \mathbf{s}\right)},
$$

sendo $z^{\prime}$ uma variável de integração conveniente e $H(z ; \mathbf{s})$ dado pela equação (4.8).

Para vincular os parâmetros cosmológicos livres do modelo, utilizamos a amostra do catálogo Union (Kowalski et al., 2008), contendo 307 supernovas do Tipo Ia.

O melhor ajuste para o conjunto de parâmetros $\mathbf{s}$ foi estimado através do uso de uma estatística de $\chi^{2}$, com

$$
\chi^{2}=\sum_{i=1}^{N} \frac{\left[\mu_{p}^{i}(z \mid \mathbf{s})-\mu_{o}^{i}(z)\right]^{2}}{\sigma_{i}^{2}},
$$

onde $\mu_{p}^{i}(z \mid \mathbf{s})$ é dado pela equação (4.17), $\mu_{o}^{i}(z)$ é o módulo de distância (com extinção corrigida) para uma dada SN Ia em $z_{i}$, e $\sigma_{i}$ é a incerteza individual do módulo de distância.

$\mathrm{Na}$ análise conjunta com a marginalização do parâmetro $h\left(H_{0}=100 h \mathrm{~km} \mathrm{~s}^{-1} \mathrm{Mpc}^{-1}\right)$, encontramos $\alpha=0.93_{-0.26-0.44-0.63}^{+0.22+0.35+0.46}$ e $\Omega_{m}=1.34_{-0.40-0.68-0.98}^{+0.34+0.54+0.72}$ com $68.3 \%, 95.4 \%$ e $99.7 \%$ de confiança estatística, respectivamente, com $\chi_{\min }^{2}=310.23$ e $\nu=305$ graus de liberdade. $\mathrm{O}$ valor encontrado do $\chi^{2}$ reduzido, $\chi_{r}^{2}=\chi_{\min }^{2} / \nu$, foi $\chi_{r}^{2}=1.017$, mostrando portanto que o modelo fornece um ajuste muito bom para os dados e que um universo fechado, dominado apenas por bárions e matéria escura fria, é favorecido por este conjunto de dados.

Na figura 4.1a, mostramos o espaço de parâmetros $\Omega_{m e f f} \times \alpha$. O melhor ajuste para o parâmetro de densidade de matéria efetivo é $\Omega_{\text {meff }}=\Omega_{m}-\alpha=0.41_{-0.15-0.26-0.37}^{+0.13+0.21+0.29} \mathrm{com}$, respectivamente, $68.3 \%, 95.4 \%$ e $99.7 \%$ de confiança estatística . Na figura 4.1b, mostramos as curvas de confiança obtidas para o espaço de parâmetros $\Omega_{m} \times \alpha$. Os resultados obtidos foram $\Omega_{m}=1.34_{-0.40-0.68-0.98}^{+0.34+0.54+0.72}$ e $\alpha=0.93_{-0.26-0.44-0.63}^{+0.22+0.35+0.46}$, mostrando que o melhor ajuste favorece um modelo de universo fechado, mas compatível com o modelo plano dentro de $1 \sigma$. 

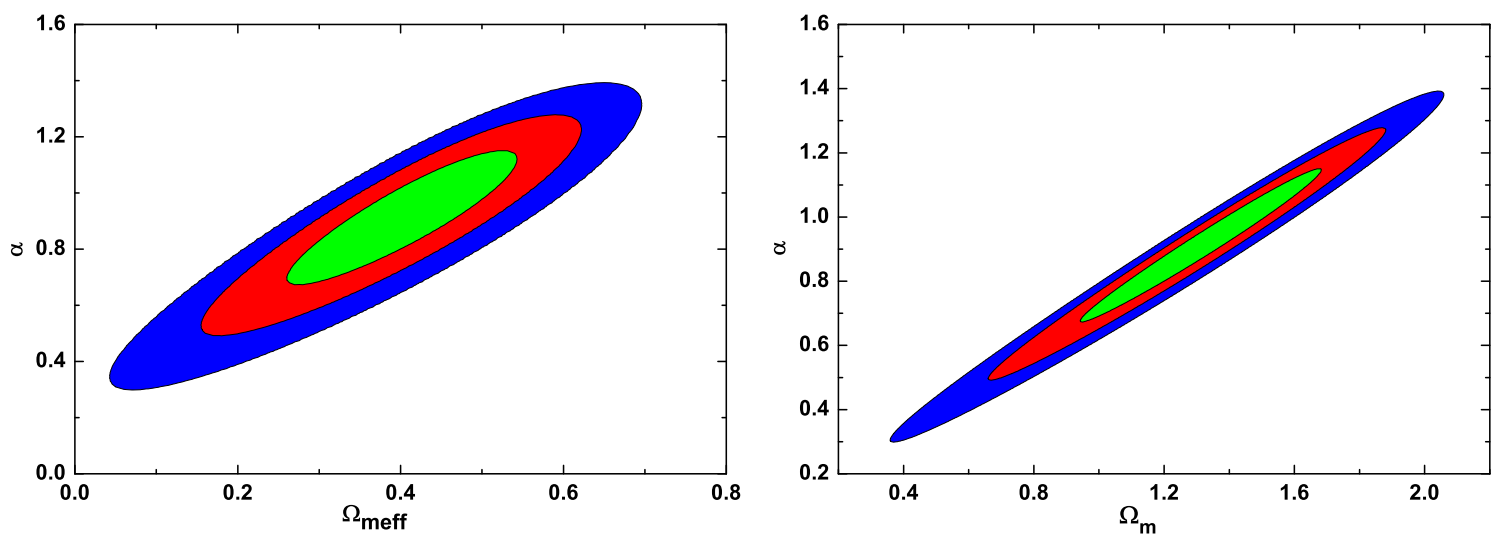

Figura 4.1: a) Plano $\left(\Omega_{m e f f} \times \alpha\right)$ para o modelo CCDM, com base nos 307 dados de Supernovas do catálogo Union (Kowalski et al., 2008), mostrando os vínculos para $68.3 \%$, 95.4\% e $99.7 \%$ c.e. para os dois parâmetros livres . b) Plano $\left(\Omega_{m} \times \alpha\right)$ para o modelo CCDM mostrando os vínculos para $68.3 \%$, $95.4 \%$ e $99.7 \%$ c.e. para os dois parâmetros livres. Note que, assim como no modelo $\Lambda$ CDM, estes dados favorecem um universo fechado com criação de partículas de matéria escura fria $\left(\Omega_{m}=1.34_{-0.68}^{+0.54}\right.$, em $\left.2 \sigma\right)$.

No caso plano, o único parâmetro livre é $\alpha$, e, como o esperado, o modelo fornece um bom ajuste aos dados de SNe Ia. Nesta análise encontramos que $\alpha=0.713_{-0.028-0.058-0.089}^{+0.027+0.052+0.077}$, com $\chi_{\min }^{2}=311.94$ e $\chi_{r}^{2}=1.019$, para 306 graus de liberdade (figura 4.2). Este excelente ajuste confirma que podemos explicar os dados de SNe Ia através do modelo constituído apenas por matéria não-relativística e criação de partículas de CDM. Os parâmetros ajustados para os modelos CCDM com curvatura e CCDM plano estão resumidos na tabela $(4.1)$

Tabela 4.1 - Modelo CCDM

\begin{tabular}{||c|c|c||}
\hline \hline Parâmetro & CCDM & CCDM - plano \\
\hline$\Omega_{m}$ & $1.34_{-0.40-0.68-0.98}^{+0.34+0.54+0.72}$ & 1 \\
$\alpha$ & $0.93_{-0.26-0.44-0.63}^{+0.22+0.35+0.46}$ & $0.713_{-0.028-0.058-0.089}^{+0.027+0.052+0.077}$ \\
\hline
\end{tabular}

Podemos agora passar para a análise do redshift de transição, $z_{t}$.

Primeiramente, observemos que a combinação entre as equações (3.10) e (3.11) resulta em

$$
\frac{\ddot{a}}{a}=-\frac{4 \pi G}{3}\left(\rho_{b}+\rho_{d m}+3 p_{c}\right)
$$

Lembrando que a pressão de criação toma a forma (4.3), vemos que 


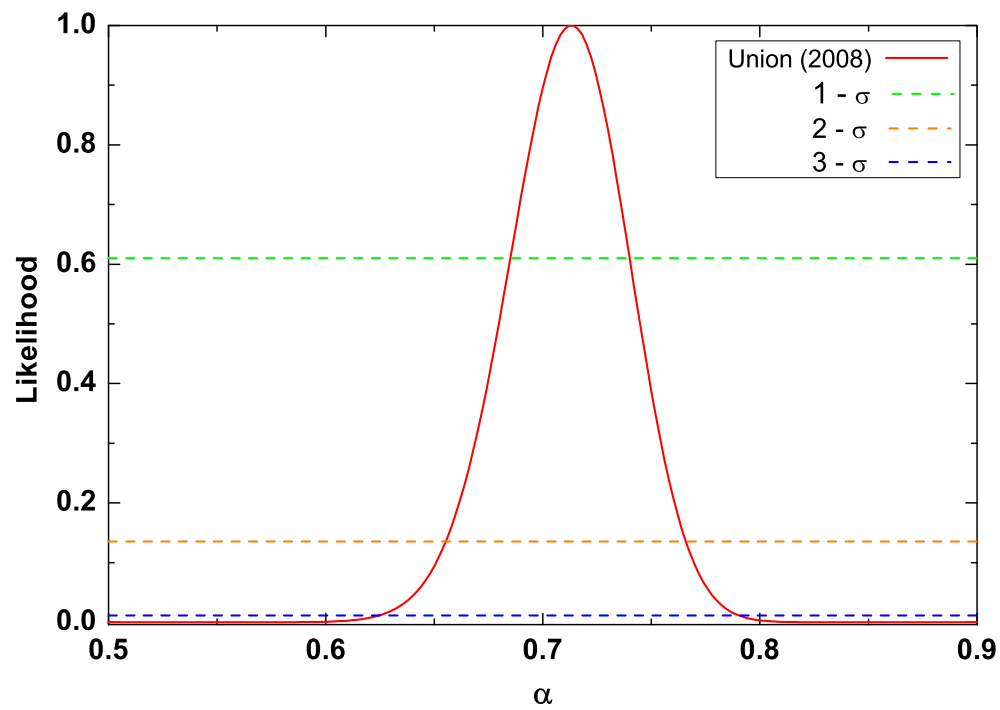

Figura 4.2: A likelihood do parâmetro livre $\alpha$ para o modelo CCDM plano calculada através dos dados de supernovas tipo Ia do Union (Kowalski et al., 2008)). O parâmetro $\alpha$ é vinculado como $\alpha=0.713_{-0.028-0.058-0.089}^{+0.027+0.052+0.077}$

$$
\frac{\ddot{a}}{a}=-\frac{4 \pi G}{3 \rho_{c 0}}\left[\left(\Omega_{b}+\Omega_{d m}-\alpha\right)(1+z)^{3}-2 \alpha\right]
$$

Igualando a expressão (4.21) a zero, encontramos a seguinte equação para o redshift de transição

$$
z_{t}=\left(\frac{2 \alpha}{\Omega_{m}-\alpha}\right)^{1 / 3}-1 .
$$

O valor do redshift de transição é implicitamente dependente do parâmetro de curvatura. Para o modelo CCDM curvo, utilizando-se os melhores ajustes de $\alpha$ e $\Omega_{m}$ na expressão (4.22), obtemos o valor central $z_{t}=0.65$. No caso CCDM plano o redshift de transição é um pouco maior $\left(z_{t}=0.71\right)$ de acordo com o fato de que, neste último caso, temos menos matéria. Na figura 4.3 mostramos a likelihood para o caso plano.

\subsection{Comentários Finais}

No contexto da Teoria da Relatividade Geral, a expansão acelerada do universo é um fato observacional muito bem consolidado. Usualmente, este estágio acelerado é explicado através da existência de um fluido exótico, chamado de energia escura, que tem como 


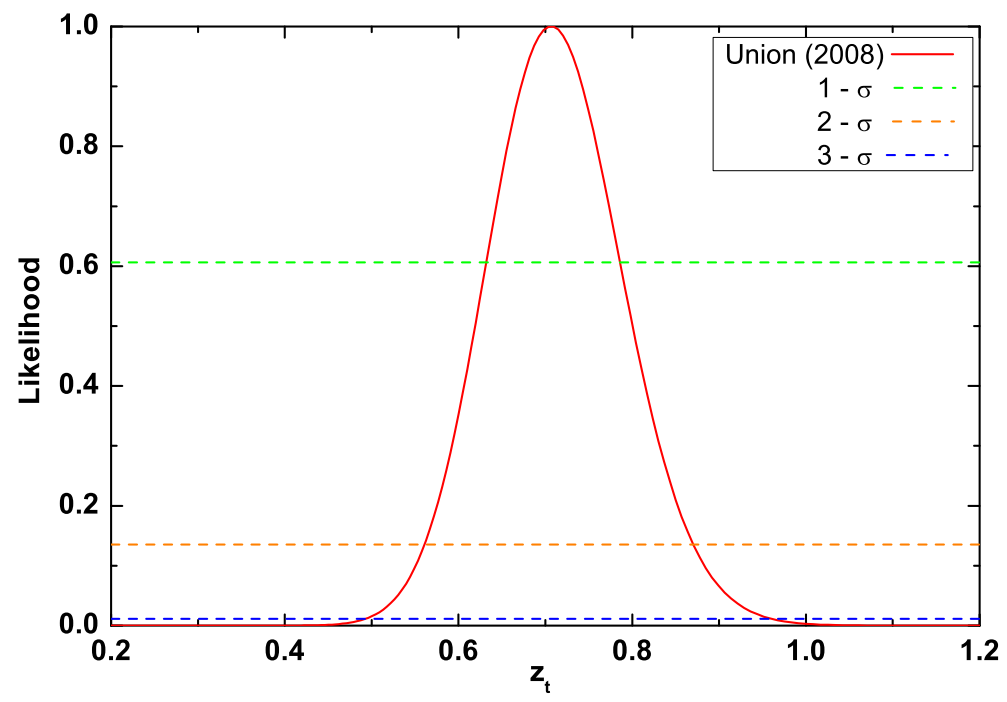

Figura 4.3: A likelihood do redshift de transição $\left(z_{t}\right)$ para o modelo CDM plano calculado através dos dados de supernovas tipo Ia do Union (Kowalski et al., 2008)). O redshift de transição é vinculado como $z_{t}=0.706_{-0.074-0.15-0.21}^{+0.080+0.16+0.26}$.

principal propriedade a pressão negativa. Na literatura existem múltiplas alternativas possíveis representando fenomenologicamente esta componente escura.

Porém, como vimos neste capítulo e no anterior, mecanismos em que há criação de matéria escura fria às custas do campo gravitacional são capazes de dar conta das observações da aceleração cósmica, com a vantagem adicional de reduzirmos o setor escuro do universo a apenas uma componente e sem romper com a TRG, a melhor teoria de gravitação disponível.

O ponto crucial dos modelos de criação de matéria escura é a determinação de $\Gamma$, a taxa de criação. Em investigações anteriores, mostrou-se que nos modelos em que $\Gamma \propto H$, não há transição entre os regimes de expansão desacelerada e acelerada. Já nos modelos em que se definiu a taxa de criação como $\Gamma=3 \beta H, \operatorname{com} \beta$ uma função do tempo, existe a transição entre os regimes mas, além das dificuldades matemáticas encontradas, os vínculos obtidos em altos e baixos redshifts mostraram-se incoerentes. Além disto, em geral os modelos com criação possuem outra importante dificuldade pois são planos, constituídos apenas por matéria escura e bariônica, mas necessitam ser compatíveis com as observações fornecidas por aglomerados $\left(\Omega_{b}+\Omega_{d m} \approx 0.3\right)$.

Neste capítulo, mostramos que tais dificuldades podem ser superadas através da escolha da taxa de criação mostrada na equação (4.2). No modelo proposto, o modelo CCDM, 
mostramos que as equações dinâmicas que regem a evolução do fator de escala são exatamente as mesmas que a do modelo de concordância cósmica. Ressaltamos ainda que não é necessário que o valor da constante de Hubble seja pequeno para que o universo tenha idade suficiente para acomodar suas estruturas mais antigas.

Investigamos ainda como os dados de supernovas tipo Ia vinculam os parâmetros do modelo CCDM nos casos plano e curvo. Para isto, realizamos o teste de $\chi^{2}$ utilizando a amostra Union (Kowalski et al., 2008), contendo os dados de 307 SN Ia. Os vínculos sobre os parâmetros $\alpha$ e $\Omega_{m}$ foram obtidos marginalizando a constante de Hubble, $H_{0}$. No caso curvo, os valores vinculados foram $\alpha=0.93_{-0.26-0.44-0.63}^{+0.22+0.35+0.46} \Omega_{m}=1.34_{-0.40-0.68-0.98}^{+0.34+0.54+0.72}$, com $\chi_{r}^{2}=1.017$, favorecendo um universo com curvatura positiva. No caso plano, o ajuste obtido foi $\alpha=0.713_{-0.028-0.058-0.089}^{+0.027+0.052+0.077}$, com $\chi_{r}^{2}=1.019$.

Concluindo, este modelo constituído apenas por matéria não-relativística mostrou-se capaz de explicar a evidência de aceleração assim como resolver o problema da idade do universo. Os ajustes obtidos para os dados de SNe Ia foram tão bons quanto os obtido no modelo padrão. Outros testes envolvendo observações astronômicas ainda devem ser realizados. Esperamos ainda investigar detalhadamente o papel do parâmetro de densidade de matéria escura efetivo, $\Omega_{m e f f}$, que poderá ser fundamental na conciliação entre as observações de formação de estruturas e o modelo CCDM. 


\section{Capítulo 5}

\section{Conclusão}

Um dos problemas centrais da Cosmologia contemporânea é fornecer uma explicação fisicamente consistente e rigorosa da atual fase acelerada do universo. Dentro do paradigma da Teoria da Relatividade Geral (TRG), o meio mais simples de acelerar o universo é através da inclusão de uma componente escura extra (em adição à matéria escura), usualmente denominada de energia escura. A principal característica exigida para esta nova componente é ter pressão negativa pois, devido a um efeito puramente relativístico, uma pressão negativa é capaz de acelerar o universo.

Admitindo a validade da TRG na descrição dos fenômenos físicos nas escalas pertinentes à Cosmologia, existem múltiplas alternativas de fluidos exóticos que podem contribuir com a aceleração. A possibilidade mais simples é a constante cosmológica que, em sua interpretação moderna, corresponde à densidade de energia do vácuo. Embora esta alternativa forneça excelentes ajustes aos dados observacionais, sua principal fraqueza se encontra no chamado problema da constante cosmológica, isto é, na enorme discrepância entre as estimativas observacionais e teóricas da densidade de energia do vácuo.

Nesta dissertação, propomos um novo modelo cosmológico acelerado pela criação de matéria escura fria (Creation of Cold Dark Matter - CCDM) . Este modelo, recentemente dominado por matéria escura fria, não requer a energia escura para explicar as observações cosmológicas. Neste caso, o parâmetro de densidade de energia do vácuo é nulo $\left(\Omega_{\Lambda}=0\right)$ e, portanto, o problema da constante cosmológica não é considerado.

A recente aceleração é alimentada neste trabalho por um processo irreversível de criação de partículas e, embora o modelo seja formado apenas por matéria não relativística, o valor de $H_{0}$ não precisa ser pequeno para resolver o problema da idade. 
Tabela $5.1-\Lambda$ CDM vs. CCDM

\begin{tabular}{cc}
\hline \hline$\Lambda \mathrm{CDM}$ & $\mathrm{CCDM}$ \\
\hline$\Omega_{\Lambda}$ & $\alpha$ \\
$\Omega_{m}$ & $\Omega_{m e f f} \equiv \Omega_{m}-\alpha$ \\
Vácuo DE & criação de CDM \\
Aceleração $\left(z_{t} \approx 0.71, k=0\right)$ & Aceleração $\left(z_{t} \approx 0.71, k=0\right)$
\end{tabular}

Também é importante mencionar a equivalência dinâmica entre a cosmologia CCDM e a $\Lambda$ CDM a nível das equações de background. Na verdade, o cenário CCDM pode ser formalmente interpretado como um fluido com mistura de duas componentes: a matéria sem pressão, com parâmetro de densidade $\Omega_{m e f f}=\Omega_{m}-\alpha$, mais uma componente de vácuo com $\rho_{v}=-p_{v}=\alpha \rho_{c 0}$, onde $\rho_{c 0}$ é a densidade crítica do universo hoje.

$\mathrm{Na}$ tabela (5.1), apresentamos uma comparação qualitativa entre as duas abordagens. Vemos que para o caso CCDM não-plano, temos dois parâmetros dinâmicos, a saber, $\alpha \mathrm{e}$ $\Omega_{m}$ (ou $\Omega_{m e f f}$ ), equivalentemente ao modelo $\Lambda \mathrm{CDM}$ com curvatura, cujo os parâmetros dinâmicos são $\Omega_{\Lambda}$ e $\Omega_{m}$.

Já no caso CCDM plano, há somente um parâmetro dinâmico livre (exatamente como no modelo $\Lambda \mathrm{CDM}$ plano), isto é, $\alpha$. Esta equivalência formal explica como os cenários CCDM conseguem prover um excelente ajuste às supernovas tipo Ia (c.f. texto e figuras 4.1 e 4.2). Desta forma, pode-se dizer que a cosmologia $\Lambda$ CDM é uma das possíveis descrições efetivas de cenários do tipo CCDM.

Por outro lado, uma vez que o mecanismo de criação adotado aqui é descrito classicamente como um processo irreversível (Prigogine et al., 1989; Lima et al., 1991; Calvão et al., 1992), o problema básico desta nova cosmologia está relacionado à ausência de uma abordagem consistente baseada na teoria quântica de campos em espaços curvos. De qualquer forma, os estudos das últimas décadas relacionados ao problema da constante cosmológica sugerem que as possíveis dificuldades na busca de um formalismo quântico mais rigoroso para a criação de matéria em um universo em expansão são muito menores que o problema da constante cosmológica. De fato, os mecanismos básicos vêm sendo discutidos há bastante tempo (Parker, 1968; Fulling et al., 1974; Birrell e Davies, Birrell e Davies; Grib 
et al., 1984; Mukhanov e Winitzki, Mukhanov e Winitzki), de forma que o problema está reduzido a como levar em conta a taxa de produção de entropia adequadamente, presente no mecanismo de criação associado à pressão de criação

Finalmente, para aqueles que acreditam que o modelo $\Lambda$ CDM contém toda a física que precisamos para confrontar a próxima geração de testes cosmológicos, chamamos a atenção para o modelo proposto aqui. Ele é simples como o $\Lambda$ CDM, possui a mesma dinâmica, e, o mais importante, se baseia apenas na existência de uma componente escura, a saber, a matéria escura, cujo o status é relativamente maior que o da energia escura. Naturalmente, novos vínculos sobre os parâmetros relevantes $\left(\alpha\right.$ e $\left.\Omega_{m}\right)$ obtidos por observações complementares precisam ser investigados com o intuito de descobrir se o modelo CCDM proposto aqui fornece uma descrição realística do universo observado. Nesse sentido, como foi discutido recentemente por Basilakos e Lima (2009), a parte da matéria que efetivamente aglomera no modelo CCDM está em acordo com as recentes observações em grandes escalas e o mesmo acontece com as oscilações acústicas dos baryons e o chamado shift parameter.

A princípio, testes adicionais medindo o espectro de potência da matéria e das anisotropias da radiação cósmica de fundo, a influência de galáxias e aglomerados produzindo distorções e/ou imagens múltiplas de fontes distantes (lentes gravitacionais), como também o teste da fração de massa do gás em aglomerados, poderão decidir entre as cosmologias $\Lambda$ CDM e CCDM. No futuro, pretendemos investigar novos limites sobre os parâmetros CCDM obtidos pelas equações cosmológicas perturbadas e de background, e também fazer uma comparação detalhada com as previsões do modelo $\Lambda$ CDM. 


\section{Referências Bibliográficas}

Abdel-Rahman A., Singularity-free decaying-vacuum cosmologies, Phys. Rev. D, 1992, vol. 45 , p. 3497

Alcaniz J. S., Jain D., Dev A., High-redshift objects and the generalized Chaplygin gas, Phys. Rev. D, 2003, vol. 67, p. 043514

Alcaniz J. S., Lima J. A. S., New Limits on $\Omega_{\Lambda}$ and $\Omega_{M}$ from Old Galaxies at High Redshift, ApJ, 1999, vol. 521, p. L87

Alcaniz J. S., Lima J. A. S., Dark Energy and the Epoch of Galaxy Formation, ApJ, 2001, vol. 550, p. L133

Alcaniz J. S., Lima J. A. S., Cunha J. V., Cosmological implications of APM 08279+5255, an old quasar at $\mathrm{z}=3.91$, MNRAS, 2003, vol. 340, p. L39

Alcaniz J. S., Maia J. M., Current and future supernova constraints on decaying $\Lambda$ cosmologies, Phys. Rev. D, 2003, vol. 67, p. 043502

Allen S. W., Cosmological constraints from Chandra observations of galaxy clusters, Royal Society of London Philosophical Transactions Series A, 2002, vol. 360, p. 2005

Alpher R. A., Bethe H., Gamow G., The Origin of Chemical Elements, Physical Review, 1948, vol. 73 , p. 803

Alpher R. A., Herman R., Gamow G. A., Thermonuclear Reactions in the Expanding Universe, Physical Review, 1948, vol. 74, p. 1198 
Amendola L., Finelli F., Burigana C., Carturan D., WMAP and the Generalized Chaplygin Gas, JCAP, 2003, vol. 0307, p. 005

Arbab A. I., Abdel-Rahman A., Nonsingular cosmology with a time-dependent cosmological term, Phys. Rev. D, 1994, vol. 50, p. 7725

Avelino P. P., Beça L. M., de Carvalho J. P., Martins C. J., Pinto P., Alternatives to quintessence model building, Phys. Rev. D, 2003, vol. 67, p. 023511

Barrow J. D., The deflationary universe: An instability of the de Sitter universe, Physics Letters B, 1986, vol. 180, p. 335

Barrow J. D., String-driven inflationary and deflationary cosmological models, Nuclear Physics B, 1988, vol. 310, p. 743

Basilakos S., Lima J. A. S., Constraints on Cold Dark Matter Accelerating Cosmologies and Cluster Formation, arXiv:astro-ph/1003.5754v1, 2009

Bean R., Melchiorri A., Current constraints on the dark energy equation of state, Phys. Rev., 2002, vol. D65, p. 041302

Beesham A., Cosmological models with a variable cosmological term and bulk viscous models, Phys. Rev. D, 1993, vol. 48, p. 3539

Begeman K. G., Broeils A. H., Sanders R. H., Extended rotation curves of spiral galaxies - Dark haloes and modified dynamics, MNRAS, 1991, vol. 249, p. 523

Bento M. C., Bertolami O., Sen A. A., Generalized Chaplygin gas, accelerated expansion, and dark-energy-matter unification, Phys. Rev. D, 2002, vol. 66, p. 043507

Bento M. C., Bertolami O., Sen A. A., Generalized Chaplygin gas and cosmic microwave background radiation constraints, Phys. Rev. D, 2003a, vol. 67, p. 063003

Bento M. C., Bertolami O., Sen A. A., Letter: Generalized Chaplygin Gas Model: Dark Energy-Dark Matter Unification and CMBR Constraints, General Relativity and Gravitation, 2003b, vol. 35, p. 2063 
Bilic N., Tupper G. B., Viollier R. D., Unification of dark matter and dark energy: The inhomogeneous Chaplygin gas, Phys. Lett., 2002, vol. B535, p. 17

Birkel M., Sarkar S., Nucleosynthesis bounds on a time-varying cosmological _constant_, Astroparticle Physics, 1997, vol. 6, p. 197

Birrell N. D., Davies P. C. W., Quantum Fields in Curved Space

Bradač M., Allen S. W., Treu T., Ebeling H., Massey R., Morris R. G., von der Linden A., Applegate D., Revealing the Properties of Dark Matter in the Merging Cluster MACS J0025.4-1222, ApJ, 2008, vol. 687, p. 959

Bronstein M. P., The problem of the possible theory of the World as a whole, Uspekhi Astronomicheskih Nauk, 1933, Coll(Issue).3, pp. 3-30 (Russian title: K voprosu o vozmojnoi teorii mira kak tselogo), 1933, vol. 3, p. 3

Burbidge E. M., Burbidge G. R., Fowler W. A., Hoyle F., Synthesis of the Elements in Stars, Reviews of Modern Physics, 1957, vol. 29, p. 547

Caldwell R. R., An introduction to quintessence., Brazilian Journal of Physics, 2000, vol. 30 , p. 215

Caldwell R. R., Kamionkowski M., Weinberg N. N., Phantom Energy: Dark Energy with $\omega<-1$ Causes a Cosmic Doomsday, Physical Review Letters, 2003, vol. 91, p. 071301

Caldwell R. R., Steinhardt P. J., Imprint of gravitational waves in models dominated by a dynamical cosmic scalar field, Phys. Rev. D, 1998, vol. 57, p. 6057

Caldwell, R. R. A phantom menace? Cosmological consequences of a dark energy component with super-negative equation of state, Physics Letters B, 2002, vol. 545, p. 23

Calvão M. O., Waga I., Lima J. A. S., On the thermodynamics of matter creation in cosmology., Physics Letters A, 1992, vol. 162, p. 223

Carroll S. M., Hoffman M., Trodden M., Can the dark energy equation-of-state parameter w be less than -1?, Phys. Rev. D, 2003, vol. 68, p. 023509 
Carturan D., Finelli F., Cosmological Effects of a Class of Fluid Dark Energy Models, Phys. Rev., 2003, vol. D68, p. 103501

Carvalho F. C., Alcaniz J. S., Lima J. A. S., Silva R., Scalar-Field-Dominated Cosmology with a Transient Acceleration Phase, Physical Review Letters, 2006, vol. 97, p. 081301

Casimir H. B. G., On the Attraction Between Two Perfectly Conducting Plates, Indag. Math., 1948, vol. 10, p. 261

Chae K. H., et al., Constraints on cosmological parameters from the analysis of the Cosmic Lens All Sky Survey radio-selected gravitational lens statistics, Phys. Rev. Lett., 2002a, vol. 89 , p. 151301

Chae K. H., et al., Constraints on cosmological parameters from the analysis of the Cosmic Lens All Sky Survey radio-selected gravitational lens statistics, Phys. Rev. Lett., 2002b, vol. 89 , p. 151301

Chen W., Wu Y., Implications of a cosmological constant varying as $R^{-2}$, Phys. Rev. D, 1990, vol. 41, p. 695

Chiba T., Okabe T., Yamaguchi M., Kinetically driven quintessence, Phys. Rev. D, 2000, vol. 62 , p. 023511

Chiba T., Sugiyama N., Nakamura T., Cosmology with x-matter, Mon. Not. Roy. Astron. Soc., 1997, vol. 289, p. L5

Clowe D., Bradač M., Gonzalez A. H., Markevitch M., Randall S. W., Jones C., Zaritsky D., A Direct Empirical Proof of the Existence of Dark Matter, ApJ, 2006, vol. 648, p. L109

Cole S., Percival W. J., Peacock J. A., Norberg P., et al. The 2dF Galaxy Redshift Survey: power-spectrum analysis of the final data set and cosmological implications, MNRAS, 2005, vol. 362 , p. 505

Copi C. J., Schramm D. N., Turner M. S., Big bang nucleosynthesis and the baryon density of the universe, Science, 1995, vol. 267, p. 192 
Corasaniti P. S., Copeland E. J., Constraining the quintessence equation of state with SnIa data and CMB peaks, Phys. Rev. D, 2002, vol. 65, p. 043004

Cunha J. V., Alcaniz J. S., Lima J. A., Deflationary cosmology: Constraints from angular size and ages of globular clusters, Phys. Rev. D, 2002a, vol. 66, p. 023520

Cunha J. V., Alcaniz J. S., Lima J. A. S., Cosmological constraints on Chaplygin gas dark energy from galaxy cluster x-ray and supernova data, Phys. Rev. D, 2004, vol. 69, p. 083501

Daly R. A., Guerra E. J., Quintessence, Cosmology, and Fanaroff-Riley Type IIb Radio Galaxies, AJ, 2002, vol. 124, p. 1831

de Sitter W., Einstein's theory of gravitation and its astronomical consequences. Third paper, MNRAS, 1917, vol. 78, p. 3

Dev A., Alcaniz J. S., Jain D., Cosmological consequences of a Chaplygin gas dark energy, Phys. Rev. D, 2003, vol. 67, p. 023515

Dicke R. H., Peebles P. J. E., Roll P. G., Wilkinson D. T., Cosmic Black-Body Radiation., ApJ, 1965, vol. 142, p. 414

Durrer R., The cosmic microwave backgrond, PoS, 2007, vol. CARGESE2007, p. 006

Efstathiou G., Constraining the equation of state of the Universe from Distant Type Ia Supernovae and Cosmic Microwave Background Anisotropies, 1999

Einstein A., Kosmologische Betrachtungen zur allgemeinen Relativitätstheorie, Sitzungsberichte der Königlich Preußischen Akademie der Wissenschaften (Berlin), Seite 142-152., 1917, pp 142-152

Einstein A., de Sitter W., On the Relation between the Expansion and the Mean Density of the Universe, Proceedings of the National Academy of Science, 1932, vol. 18, p. 213

Erickson J. K., Caldwell R. R., Steinhardt P. J., Armendariz-Picon C., Mukhanov V. F., Measuring the speed of sound of quintessence, Phys. Rev. Lett., 2002, vol. 88, p. 121301 
Fabris J. C., Goncalves S. V. B., de Souza P. E., Fitting the Supernova Type Ia Data with the Chaplygin Gas, arXiv:astro-ph/0207430, 2002

Fomin P. I., Gravitational instability of a vacuum and the cosmological problem, Akademiia Nauk Ukrains koi RSR Dopovidi Seriia Fiziko Matematichni ta Tekhnichni Nauki, 1975, pp 831-835

Forman W., Jones C., X-ray-imaging observations of clusters of galaxies, Ann. Rev. Astron. Astrophys., 1982, vol. 20, p. 547

Freedman W. L., Madore B. F., Gibson B. K., Ferrarese L., Kelson D. D., Sakai S., Mould J. R., Kennicutt Jr. R. C., Ford H. C., Graham J. A., Huchra J. P., Hughes S. M. G., Illingworth G. D., Macri L. M., Stetson P. B., Final Results from the Hubble Space Telescope Key Project to Measure the Hubble Constant, ApJ, 2001, vol. 553, p. 47

Freese K., Adams F. C., Frieman J. A., Mottola E., Cosmology with decaying vacuum energy, Nuclear Physics B, 1987, vol. 287, p. 797

Friedmann A., Über die Krümmung des Raumes, Zeitschrift für Physik, 1922, vol. 10, p. 377

Friedmann A., Über die Möglichkeit einer Welt mit konstanter negativer Krümmung des Raumes, Zeitschrift für Physik, 1924, vol. 21, p. 326

Fulling S. A., Parker L., Hu B. L., Particle Creation in Expanding Universes, Phys. Rev. D, 1974, vol. 10, p. 3905

Gamow G., Expanding Universe and the Origin of Elements, Physical Review, 1946, vol. 70, p. 572

Garnavich P. M., et al., Supernova Limits on the Cosmic Equation of State, Astrophys. J., 1998, vol. 509, p. 74

González-Díaz P. F., Sigüenza C. L., Phantom thermodynamics, Nuclear Physics B, 2004, vol. 697, p. 363

Grib A. A., Mamayev S. G., Mostepanenko V. M., Self-Consistent Treatment of Vacuum Quantum Effects in Isotropic Cosmology. In Quantum Gravity , 1984, p. 197 
Gurvits L. I., Kellermann K. I., Frey S., The "angular size - redshift" relation for compact radio structures in quasars and radio galaxies, A\&A, 1999, vol. 342, p. 378

Hamuy M., Phillips M. M., Suntzeff N. B., Schommer R. A., Maza J., Aviles R., The Hubble Diagram of the Calan/Tololo Type IA Supernovae and the Value of $H_{O}$, AJ, 1996, vol. 112 , p. 2398

Hannestad S., Mortsell E., Probing the dark side: Constraints on the dark energy equation of state from CMB, large scale structure and Type Ia supernovae, Phys. Rev., 2002, vol. D66, p. 063508

Hicken M., Wood-Vasey W. M., Blondin S., Challis P., Jha S., Kelly P. L., Rest A., Kirshner R. P., Improved Dark Energy Constraints from 100 New CfA Supernova Type Ia Light Curves, ApJ, 2009, vol. 700, p. 1097

Hoyle F., A New Model for the Expanding Universe, MNRAS, 1948, vol. 108, p. 372

$\mathrm{Hu}$ W., Dodelson S., Cosmic Microwave Background Anisotropies, Ann. Rev. Astron. Astrophys., 2002, vol. 40, p. 171

Hubble E., A relation between distance and radial velocity among extra-galactic nebulae, Proc. Nat. Acad. Sci., 1929, vol. 15, p. 168

Jain D., Dev A., Panchapakesan N., Mahajan S., Bhatia V. B., Gravitational lensing constraint on the cosmic equation of state, Int. J. Mod. Phys., 2003, vol. D12, p. 953

Kamenshchik A., Moschella U., Pasquier V., An alternative to quintessence, Physics Letters B, 2001, vol. 511, p. 265

Kirkman D., Tytler D., Suzuki N., O’Meara J. M., Lubin D., The Cosmological Baryon Density from the Deuterium-to-Hydrogen Ratio in QSO Absorption Systems: D/H toward Q1243+3047, ApJS, 2003, vol. 149, p. 1

Kolb E. W., Turner M. S., The Early universe, Front. Phys., 1990, vol. 69, p. 1

Komatsu E., Dunkley J., Nolta M. R., Bennett C. L., Gold B., Hinshaw G., Jarosik N., Larson D., Limon M., Page L., Spergel D. N., Halpern M., Hill R. S., Kogut A., 
Meyer S. S., Tucker G. S., Weiland J. L., Wollack E., Wright E. L., Five-Year Wilkinson Microwave Anisotropy Probe (WMAP) Observations: Cosmological Interpretation, arXiv:astro-ph/0803.0547, 2008

Komatsu E., Smith K. M., Dunkley J., et al. Seven-Year Wilkinson Microwave Anisotropy Probe (WMAP) Observations: Cosmological Interpretation, arXiv:astro-ph/1001.4538, 2010

Kowalski M., Rubin D., Aldering G., et al. Improved Cosmological Constraints from New, Old, and Combined Supernova Data Sets, ApJ, 2008, vol. 686, p. 749

Krauss L. M., Old Galaxies at High Redshift and the Cosmological Constant, ApJ, 1997, vol. 480 , p. 466

Kujat J., Linn A. M., Scherrer R. J., Weinberg D. H., Prospects for Determining the Equation of State of the Dark Energy: What can be Learned from Multiple Observables?, Astrophys. J., 2002, vol. 572, p. 1

Landau L. D., Lifshitz E. M., The classical theory of fields. Course of theoretical physics Pergamon International Library of Science, Technology, Engineering and Social Studies, Oxford: Pergamon Press, 1971, 3rd rev. engl. edition, 1971

Larson D., Dunkley J., Hinshaw et al. Seven-Year Wilkinson Microwave Anisotropy Probe (WMAP) Observations: Power Spectra and WMAP-Derived Parameters, arXiv:astro$\mathrm{ph} / 1001.4635,2010$

Lemaitre G., Evolution of the Expanding Universe, Proceedings of the National Academy of Science, 1934, vol. 20, p. 12

Lima J. A., Cunha J. V., Alcaniz J. S., Constraining the dark energy with galaxy cluster x-ray data, Phys. Rev. D, 2003, vol. 68, p. 023510

Lima J. A. S., Thermodynamics of decaying vacuum cosmologies, Phys. Rev. D, 1996, vol. 54 , p. 2571

Lima J. A. S., Alternative Dark Energy Models: An Overview, Brazilian Journal of Physics, 2004, vol. 34, p. 194 
Lima J. A. S., Alcaniz J. S., Constraining the cosmic equation of state from old galaxies at high redshift, Mon. Not. Roy. Astron. Soc., 2000, vol. 317, p. 893

Lima J. A. S., Alcaniz J. S., Dark Energy and the Angular Size-Redshift Diagram for Milliarcsecond Radio Sources, ApJ, 2002, vol. 566, p. 15

Lima J. A. S., Alcaniz J. S., Thermodynamics, spectral distribution and the nature of dark energy, Physics Letters B, 2004, vol. 600, p. 191

Lima J. A. S., Calvão M. O., Waga I., Cosmology, Thermodynamics and Matter Creation in: Frontier Physics, Essays in Honor of Jayme Tiomno, World Scientific, 1991, p. 317

Lima J. A. S., Germano A. S. M., On the equivalence of bulk viscosity and matter creation, Physics Letters A, 1992, vol. 170, p. 373

Lima J. A. S., Germano A. S. M., Abramo L. R. W., FRW-type cosmologies with adiabatic matter creation, Phys. Rev. D, 1996, vol. 53, p. 4287

Lima J. A. S., Jesus J. F., Oliveira F. A., CDM Accelerating Cosmology as an Alternative to LCDM model, arXiv:astro-ph/0911.5727, 2009

Lima J. A. S., Maia J. M. F., Some Cosmological Consequences of a $\Lambda$-TERM Varying as $\beta H^{2}+\alpha R^{-n}$, Modern Physics Letters A, 1993, vol. 8, p. 591

Lima J. A. S., Maia J. M. F., Deflationary cosmology with decaying vacuum energy density, Phys. Rev. D, 1994, vol. 49, p. 5597

Lima J. A. S., Maia J. M. F., Pires N., Constraints from Big Bang Nucleosynthesis on a Time-Varying Cosmological Constant. In The Light Elements and their Evolution , vol. 198 of IAU Symposium, 2000, p. 111

Lima J. A. S., Portugal R., Waga I., Bulk-viscosity-driven asymmetric inflationary universe, Phys. Rev. D, 1988, vol. 37, p. 2755

Lima J. A. S., Silva F. E., Santos R. C., Accelerating cold dark matter cosmology $\left(\Omega_{\Lambda} \equiv 0\right)$, Classical and Quantum Gravity, 2008, vol. 25, p. 205006 
Lima J. A. S., Tiomno J., On the thermodynamics of one-fluid Szekeres-like cosmologies, Classical and Quantum Gravity, 1989, vol. 6, p. L93

Lima J. A. S., Trodden M., Decaying vacuum energy and deflationary cosmology in open and closed universes, Phys. Rev. D, 1996, vol. 53, p. 4280

Makler M., Quinet de Oliveira S., Waga I., Observational constraints on Chaplygin quartessence: Background results, Phys. Rev. D, 2003, vol. 68, p. 123521

McInnes B., What If $\omega<-1$ ?, arXiv:astro-ph/0210321, 2002

Mukhanov V., Winitzki S., Introduction to quantum effects in gravity

North J. D., The measure of the universe. A history of modern cosmology, 1965

Overduin J. M., Cooperstock F. I., Evolution of the scale factor with a variable cosmological term, Phys. Rev. D, 1998, vol. 58, p. 043506

Özer M., Taha M. O., A possible solution to the main cosmological problems, Physics Letters B, 1986, vol. 171, p. 363

Padmanabhan T., Choudhury T. R., A theoretician's analysis of the supernova data and the limitations in determining the nature of dark energy, Mon. Not. Roy. Astron. Soc., 2003, vol. 344, p. 823

Parker L., Particle Creation in Expanding Universes, Phys. Rev. Lett., 1968, vol. 21, p. 562

Peacock J. A., Cosmological Physics, 1999

Peebles P. J., Ratra B., The cosmological constant and dark energy, Reviews of Modern Physics, 2003, vol. 75, p. 559

Penzias A. A., Wilson R. W., A Measurement of Excess Antenna Temperature at 4080 Mc/s., ApJ, 1965, vol. 142, p. 419

Pereira S. H., Bessa C. H. G., Lima J. A. S., Quantized fields and gravitational particle creation in $\mathrm{f}(\mathrm{R})$ expanding universes, arXiv:astro-ph/0911.0622, 2009 
Perlmutter S. e. a., Measurements of Omega and Lambda from 42 High-Redshift Supernovae, ApJ, 1999, vol. 517, p. 565

Perlmutter S., Turner M. S., White M. J., Constraining dark energy with SNe Ia and large-scale structure, Phys. Rev. Lett., 1999, vol. 83, p. 670

Prigogine I., Geheniau J., Gunzig E., Nardone P., Thermodynamics and cosmology, General Relativity and Gravitation, 1989, vol. 21, p. 767

Rapetti D., Allen S. W., Mantz A., The prospects for constraining dark energy with future X-ray cluster gas mass fraction measurements, MNRAS, 2008, vol. 388, p. 1265

Riess A. G., Filippenko A. V., Challis P., et al. Observational Evidence from Supernovae for an Accelerating Universe and a Cosmological Constant, AJ, 1998, vol. 116, p. 1009

Robertson H. P., On the Foundations of Relativistic Cosmology, Proceedings of the National Academy of Science, 1929, vol. 15, p. 822

Robertson H. P., Kinematics and World-Structure II., ApJ, 1936, vol. 83, p. 187

Roos M., Dark Matter: The evidence from astronomy, astrophysics and cosmology, arXiv:astro-ph/1001.0316, 2010

Rubin V. C., Ford Jr. W. K., Rotation of the Andromeda Nebula from a Spectroscopic Survey of Emission Regions, Astrophys. J., 1970, vol. 159, p. 379

Sahni V., Shtanov Y., Braneworld models of dark energy, Journal of Cosmology and AstroParticle Physics, 2003, vol. 11, p. 14

Sahni V., Starobinsky A., The Case for a Positive Cosmological $\Lambda$-Term, International Journal of Modern Physics D, 2000, vol. 9, p. 373

Saini T. D., Raychaudhury S., Sahni V., Starobinsky A. A., Reconstructing the Cosmic Equation of State from Supernova Distances, Physical Review Letters, 2000, vol. 85, p. 1162

Santos J., Alcaniz J. S., Rebouças M. J., Carvalho F. C., Energy conditions in f(R) gravity, Phys. Rev. D, 2007, vol. 76, p. 083513 
Santos R. C., Efeitos das Inomogeneidades da Matéria em Cosmologias Aceleradas, Universidade Federal do Rio Grande do Norte, 2007, Tese de Doutorado, 198 p.

Schaefer B. E., The Hubble Diagram to Redshift greater than 6 from 69 Gamma-Ray Bursts, ApJ, 2007, vol. 660, p. 16

Schuecker P., Caldwell R. R., Bohringer H., Collins C. A., Guzzo L., Observational Constraints on General Relativistic Energy Conditions, Cosmic Matter Density and Dark Energy from X- Ray Clusters of Galaxies and Type-Ia Supernovae, Astron. Astrophys., 2003, vol. 402, p. 53

Silva P. T., Bertolami O., Expected Constraints on the Generalized Chaplygin Equation of State from Future Supernova Experiments and Gravitational Lensing Statistics, ApJ, 2003, vol. 599, p. 829

Silva R., Lima J. A. S., Calvão M. O., Temperature Evolution Law of Imperfect Relativistic Fluids, arXiv:gr-qc/0201048, 2002

Smith S., The Mass of the Virgo Cluster, ApJ, 1936, vol. 83, p. 23

Smoot G. F., et al., Structure in the COBE differential microwave radiometer first year maps, Astrophys. J., 1992, vol. 396, p. L1

Sparnaay M. J., Measurements of attractive forces between flat plates, Nature, 1957, vol. 180, p. 334

Steigman G., Primordial Nucleosynthesis For The New Millennium (Invited Paper). In The Light Elements and their Evolution, vol. 198 of IAU Symposium, 2000, p. 13

Steigman G., Primordial Nucleosynthesis:. Successes and Challenges, International Journal of Modern Physics E, 2006, vol. 15, p. 1

Steigman G., Santos R. C., Lima J. A. S., An accelerating cosmology without dark energy, Journal of Cosmology and Astro-Particle Physics, 2009, vol. 6, p. 33

Tegmark M., Blanton M. R., Strauss M. A., Hoyle F., et al. The Three-Dimensional Power Spectrum of Galaxies from the Sloan Digital Sky Survey, ApJ, 2004, vol. 606, p. 702 
Tryon E. P., Is the Universe a Vacuum Fluctuation?, Nature, 1973, vol. 246, p. 396

Tucker W., Blanco P., Rappoport S., David L., Fabricant D., Falco E. E., Forman W., Dressler A., Ramella M., 1E 0657-56: A Contender for the Hottest Known Cluster of Galaxies, ApJ, 1998, vol. 496, p. L5+

Turner M. S., Coherent scalar-field oscillations in an expanding universe, Phys. Rev. D, 1983, vol. 28 , p. 1243

Turner M. S., White M., CDM models with a smooth component, Phys. Rev. D, 1997, vol. 56, p. 4439

Turyshev S. G., Experimental Tests of General Relativity, Ann. Rev. Nucl. Part. Sci., 2008, vol. 58, p. 207

Vikhlinin A., et al., X-ray Cluster Cosmology, 2009

Vishwakarma R. G., A study of angular size-redshift relation for models in which $\Lambda$ decays as the energy density, Classical and Quantum Gravity, 2000, vol. 17, p. 3833

Waga I., Decaying vacuum flat cosmological models - Expressions for some observable quantities and their properties, apj, 1993, vol. 414, p. 436

Waga I., Bloomfield Torres L. F., Vacuum Decaying Cosmological Models and Gravitational Lensing. In Astrophysical Applications of Gravitational Lensing, vol. 173 of IAU Symposium, 1996, p. 23

Walker A. G., On Milne's theory of world structure, Proc. Lond. Math. Soc., 1936, vol. 42, p. 90

Wang L.-M., Caldwell R. R., Ostriker J. P., Steinhardt P. J., Cosmic Concordance and Quintessence, Astrophys. J., 2000, vol. 530, p. 17

Weinberg S., Entropy Generation and the Survival of Protogalaxies in an Expanding Universe, ApJ, 1971, vol. 168, p. 175

Weinberg S., Gravitation and Cosmology: Principles and Applications of the General Theory of Relativity. John Wiley \& Sons, 1972 
Weinberg S., The cosmological constant problem, Reviews of Modern Physics, 1989, vol. 61

Weinberg S., Cosmology. Oxford University Press, 2008

Wetterich C., Cosmon dark matter?, Phys. Rev., 2002, vol. D65, p. 123512

White D. A., Fabian A. C., Einstein observatory evidence for the widespread baryon over density in clusters of galaxies, Mon. Not. Roy. Astron. Soc., 1995, vol. 273, p. 72

White S. M., Navarro J. F., Evrard A. E., Frenk C. S., The baryon content of galaxy clusters: a challenge to cosmological orthodoxy, Nature, 1993, vol. 366, p. 429

Will C. M., The confrontation between general relativity and experiment, Living Rev. Rel., 2005, vol. 9, p. 3

Zel'dovich Y. B., The Cosmological constant and the theory of elementary particles, Sov. Phys. Usp., 1968, vol. 11, p. 381

Zeldovich Y. B., The Birth of a Closed Universe and the Anthropogenic Principle, Soviet Astronomy Letters, 1981, vol. 7, p. 322

Zwicky F., Die Rotverschiebung von extragalaktischen Nebeln, Helvetica Physica Acta, 1933, vol. 6, p. 110 